\title{
Model-based dose calculations for eye plaque brachytherapy
}

by

\author{
Marielle B. E. Lespérance
}

A thesis submitted to the Faculty of Graduate and Postdoctoral Affairs in partial fulfillment of the requirements for the degree of

\section{Master of Science}

in

\author{
Physics \\ Specialization in Medical Physics \\ Ottawa-Carleton Institute for Physics \\ Department of Physics \\ Carleton University \\ Ottawa, Ontario, Canada
}

September 2013

(C) 2013 Marielle B. E. Lespérance 


\section{Abstract}

Dose calculations for eye plaque brachytherapy are currently generally performed under the American Association of Physicists in Medicine TG-43 protocol with dose reported to points in water and without the effects of interseed attenuation, the plaque or ocular media on dose. Through model-based dose calculations involving a full human eye model, this work aims to develop a comprehensive understanding of dosimetry for eye plaque brachytherapy. To do so, a computational model of the human eye and surrounding tissues is developed based on geometry and composition data from the literature. Five representative models are developed for a range of photon-emitting plaques currently available and are investigated, in addition to the widely-used Collaborative Ocular Melanoma Study (COMS) plaque. Mass energy absorption and attenuation coefficients for all ocular media are calculated and compared with those for water. Monte Carlo simulations using the EGSnrc user-code BrachyDose are performed for each plaque model for three photon-emitting radionuclides, ${ }^{125} \mathrm{I},{ }^{103} \mathrm{Pd}$ and ${ }^{131} \mathrm{Cs}$. Maximum, minimum and average doses to ocular structures, isodose contours, and dose volume histograms are compared for simulations of the plaques within the full eye model and within a water phantom, and of the seeds alone in a water phantom without interseed effects (TG-43). Mass energy absorption and attenuation coefficients for ocular media differ from those for water by up to $12 \%$ for photon energies between 10 and $30 \mathrm{keV}$. Dosimetric plaque effects vary considerably with plaque model and radionuclide; for the COMS plaque in the full eye model, average doses to the tumor and lens differ from those for the plaque in water by $8-10 \%$ and $13-14 \%$, respectively, and from TG-43 simulation values by $2-17 \%$ and $29-34 \%$, respectively. The results presented herein suggest that TG-43 calculated doses are inaccurate, highlighting the need for accurate model-based dose calculations for eye plaque brachytherapy. 


\section{Statement of Originality}

This thesis contains the following work currently under review or in preparation for submission to the journal Medical Physics. Chapter 2 contains work presented in the following article: M. Lesperance, M. Inglis-Whalen, and R. Thomson, Modelbased dose calculations for COMS eye plaque brachytherapy, Med. Phys. (in press). ${ }^{1}$ Chapter 3 contains work from: M. Lesperance, M. Martinov, and R. Thomson, Modelbased dose calculations for ${ }^{125} \mathrm{I},{ }^{103} \mathrm{Pd}$, and ${ }^{131} \mathrm{Cs}$ eye plaque brachytherapy, submitted to Med. Phys.. ${ }^{2}$

Modifications to the text in both articles were made to create a unified body of work and to avoid repetition of certain methods used in both Chapters 2 and 3 of this thesis. Each manuscript was written by the author of this thesis, with revisions contributed by Dr. Rowan Thomson.

The full eye model developed in Chapter 2 was based on work performed by Matt Inglis-Whalen; his work included the development of the method used to create a rectilinear voxelized eye model from bounding surfaces and an eye model prototype. The author of this thesis used the preliminary eye model to create a new version of the eye model which includes additional ocular structures, and has modified dimensions and compositions. Further, the author of this thesis modeled tissues surrounding the eye by combining the eye model with data from the computational phantom given by the International Commission on Radiological Protection Publication $110 .^{3}$

The slotted plaque model used in Chapter 3 was developed by Martin Martinov. The specific method used to model this plaque (described in the Methods section of chapter 3) was also developed by Martin Martinov. All other plaque models, with the exception of the standardized Collaborative Ocular Melanoma Study plaque, were developed by the other of this thesis. All simulations and subsequent analysis of dose 
calculations were performed by the thesis author for all plaque models.

In addition to publishing work contained in this thesis, the work was also presented by the author at the American Association of Physicists in Medicine Annual Meeting in Indianapolis in August, 2013: M. Lesperance, M. Martinov, M. InglisWhalen, and R. Thomson, Model-based dose calculations for eye plaque brachytherapy, Med. Phys.(abstract) 40, 476 (2013). ${ }^{4}$ 


\section{Acknowledgements}

First and foremost I would like to thank my supervisor, Dr. Rowan Thomson, for all of her insight and expertise over the past two years. She has encouraged me with her positive attitude, has challenged me, and has taught me to be thorough with my research. I would also like to thank the other students in the Carleton Laboratory for Radiotherapy Physics who have helped me in various ways (with learning to code in $\mathrm{C}++$, learning how to use EGSnrc, helping with coursework, etc.) throughout my studies, especially Ali, Bryan, Nelson, Justin and Martin.

My gratitude also extends to Tyler and my family: my mother (whose genes inevitably led me to physics), my father, Sarah, and Janine, for always being supportive of my endeavors and for helping to shape who I am (whether alive or in spirit). 


\section{Contents}

Abstract

Statement of Originality iii

Acknowledgements $\quad$ V

List of Tables $\quad$ viii

List of Figures $\quad$ ix

1 Introduction 1

1.1 Brachytherapy . . . . . . . . . . . . . . . . . 1

1.2 Eye plaque brachytherapy . . . . . . . . . . . . . . . . 4

1.3 BrachyDose ......................... 8

1.4 Thesis Outline . . . . . . . . . . . . . . . . . . . 10

2 Development of an eye model and investigation of its effect on dose for the COMS plaque 13

2.1 Introduction . . . . . . . . . . . . . . . . . . . . 13

2.2 Methods . . . . . . . . . . . . . . . . . . . . . . 14

2.2.1 Development of a human eye model . . . . . . . . . . . . . . . 14

2.2.2 Monte Carlo simulations . . . . . . . . . . . . . . . . . . 21

2.3 Results and Discussion . . . . . . . . . . . . . . . . . . . . 24

2.3.1 Effect of ocular media on dose . . . . . . . . . . . . . . 24

2.3.2 Doses to ocular structures . . . . . . . . . . . . . . . 28

2.3.3 Sensitivity of doses to tumor composition, surrounding tissues, and plaque position . . . . . . . . . . . . 37 
2.3.4 Comparison of radionuclides . . . . . . . . . . . . . . 41

2.4 Conclusion . . . . . . . . . . . . . . . . . . . 44

3 Plaque effects on dose for representative plaque models in water and $\begin{array}{ll}\text { ocular media } & 46\end{array}$

3.1 Introduction . . . . . . . . . . . . . . . 46

3.2 Methods . . . . . . . . . . . . . . . . . . 47

3.2.1 Development of plaque models . . . . . . . . . . . . . . . . . 47

3.2.2 Monte Carlo simulations . . . . . . . . . . . . . . . . . . 55

3.3 Results and Discussion . . . . . . . . . . . . . . . . 57

3.3.1 Effects of eye plaques on dose distributions in water . . . . . . 57

3.3.2 Dose distributions in the eye; doses to ocular structures . . . . 64

3.3.3 Comparison of plaque models within the full eye model . . . . 72

3.4 Conclusion . . . . . . . . . . . . . . . . . . 76

$\begin{array}{lll}4 & \text { Conclusions and outlook } & 78\end{array}$

$\begin{array}{lr}\text { References } & 82\end{array}$ 


\section{List of Tables}

2.1 Elemental composition of eye structures and medium density. . . . . 18

2.2 Summary of simulation geometries and dose notation $\ldots \ldots \ldots$

2.3 COMS plaque doses to ocular structures with TG-43 normalization . 29

2.4 COMS plaque doses to ocular structures with $\% \mathrm{D}_{\text {apex }}$ normalization . 42

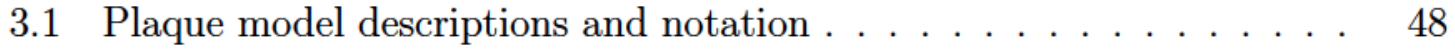

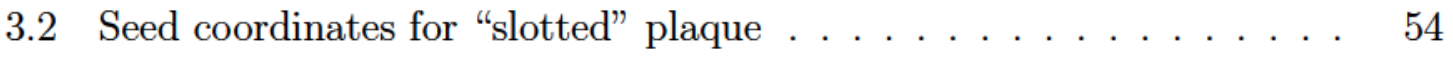

3.3 Dose to ocular structures for ${ }^{125} \mathrm{I}$ in plaques with TG-43 normalization 67

3.4 Dose to ocular structures for ${ }^{103} \mathrm{Pd}$ in plaques with TG-43 normalization 68

3.5 Dose to ocular structures for ${ }^{131} \mathrm{Cs}$ in plaques with TG-43 normalization 69

3.6 Dose to ocular structures for plaques in full eye model with $\% \mathrm{D}_{\text {apex }}$

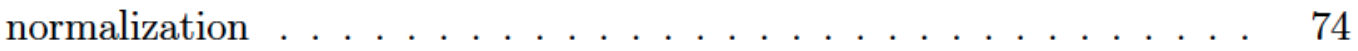




\section{List of Figures}

2.1 Bounding surfaces of eye and full voxelized eye model $\ldots \ldots \ldots \ldots$

2.2 Energy absorption and attenuation coefficients of ocular media . . . 25

2.3 COMS plaque dose ratio for full eye model to water along $z$ and $x$ axes 27

2.4 COMS plaque isodose contours with TG-43 normalization $\ldots \ldots$

2.5 COMS plaque dose volume histograms for the tumor region . . . . . 32

2.6 COMS plaque dose volume histograms for the lens region . . . . . . 34

2.7 Ratio of alternate tumor media dose to full eye model dose for ${ }^{125} \mathrm{I}$ in COMS plaque along $z$ axis $\ldots \ldots \ldots \ldots \ldots \ldots$

2.8 Ratio of varied surrounding tissue dose to full eye model dose for ${ }^{125} \mathrm{I}$ in COMS plaque along $z$ axis $\ldots \ldots \ldots \ldots \ldots$

2.9 COMS plaque isodose contours for full eye model with $\% \mathrm{D}_{\text {apex }}$ normalization . . . . . . . . . . . . . . . . . . 43

3.1 Eye plaque parameters . . . . . . . . . . . . . . . . . . . . . . 49

3.2 Representative plaque models in full eye model . . . . . . . . . 50

3.3 "Slotted" plaque seed diagram . . . . . . . . . . . . . . 53

3.4 Single seed dose ratios for plaques in water to TG-43 along $z$ axis . . 58

3.5 Dose ratios for fully-loaded plaques in water to TG-43 along $z$ axis . . 62

3.6 Dose ratios for Modulay and Silastic plaques in water to TG-43 in $z$

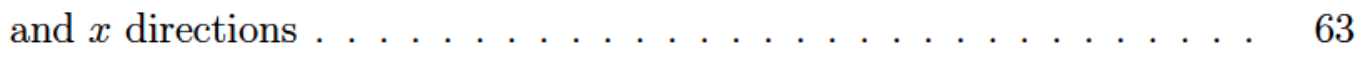

3.7 Dose ratios for fully-loaded plaques in water to TG-43 in $x$ direction . $\quad 64$

$3.8{ }^{125}$ I isodose contours for all plaque models with TG-43 normalization $\quad 66$

3.9 Isodose contours for all plaques with $\% \mathrm{D}_{\text {apex }}$ normalization $\ldots \ldots .75$ 


\section{Chapter 1}

\section{Introduction}

\subsection{Brachytherapy}

Brachytherapy is a form of cancer treatment that involves the temporary or permanent placement of radiation sources near treatment sites. This treatment often uses small radioactive seeds which are placed within a tumor (interstitial) or a nearby body cavity (intracavitary). Most brachytherapy sources are photon-emitting, although $\beta$ and neutron-emitting sources are used for certain applications. ${ }^{5}$ Photon-emitting sources are categorized as being either low-energy $(<50 \mathrm{keV})$, intermediate-energy (50-200 keV) or high-energy ( $>200 \mathrm{keV}$ ) based on the average energy of photons they emit. ${ }^{6}$ Thus, most photons emitted by brachytherapy sources generally have much lower energies than those used in external beam radiotherapy (which have energies up to to $^{5} 25 \mathrm{MeV}$ ). As a result of the low-energy photons used in brachytherapy, dose (energy absorbed per unit mass) is deposited closer to the source for brachytherapy than for external beam therapy. This allows nearby radiosensitive organs and tissues to be spared dose while delivering a sufficient dose to the tumor volume for local control. ${ }^{5}$ Another advantage to brachytherapy is that the seeds move with the tumor in regions where there is significant body movement, whereas in external beam therapy various techniques must be used to compensate for tumor motion (e.g., gating and dynamic beam tracking). ${ }^{7,8}$ Given these considerations, brachytherapy is generally used for cancers of the prostate, cervix, breast, lung and eye, among others. ${ }^{5}$

Currently, brachytherapy treatment planning is based on the protocol outlined in the Task Group 43 report (TG-43) $)^{9,10}$ by the American Association of Physicists in 
Medicine (AAPM). Using this formalism, the dose rate is determined by the following expression:

$$
\dot{D}(r, \theta)=S_{K} \cdot \Lambda \cdot \frac{G_{P}(r, \theta)}{G_{P}\left(r_{0}, \theta_{0}\right)} \cdot g_{P}(r) \cdot \phi_{a n}(r)
$$

for the one-dimensional (point source) approximation and,

$$
\dot{D}(r, \theta)=S_{K} \cdot \Lambda \cdot \frac{G_{L}(r, \theta)}{G_{L}\left(r_{0}, \theta_{0}\right)} \cdot g_{L}(r) \cdot F(r, \theta)
$$

for the two-dimensional approximation, where $S_{K}$ is the air-kerma strength (the air kerma rate in a vacuum at a given distance multiplied by the square of the distance); $\Lambda$ is the dose rate constant (the ratio of the dose rate at $1 \mathrm{~cm}$ along the transverse axis of the seed to $\left.S_{K}\right) ; r$ is the distance from the center of the seed $\left(r_{0}=1 \mathrm{~cm}\right) ; \theta$ is the polar angle measured from the longitudinal seed axis $\left(\theta_{0}=90^{\circ}\right) ; G_{P}(r, \theta)$ and $G_{L}(r, \theta)$ are the geometry functions (which give the variation of dose rate due to the geometrical distribution of the radioisotope within the seed, neglecting the effects of scatter and attenuation, and assuming point or line source distributions, respectively); $g_{P}(r)$ and $g_{L}(r)$ are the radial dose functions (which account for the effects of scatter and attenuation on dose rate as a function of $\mathrm{r})$; and $\phi_{a n}(r)$ and $F(r, \theta)$ are the anisotropy functions: in the 2-D case, $F(r, \theta)$ gives the angular dependence on the dose rate due to differences in scattering and attenuation (increased attenuation at the seed end due to the cap thickness causes this value to decrease as $\theta$ decreases), and in the 1-D case, $\phi_{a n}(r)$ is the averaged ratio of the dose rate at a distance $r$ over the full solid angle, $4 \pi$, to the dose rate at this distance at the reference angle.

Seed models are parameterized in terms of the above functions using data from measurements within water phantoms and calculations using Monte Carlo (MC) simulations. The characterization of seed models in this way allows for efficient calculation of dose distributions for multiple seeds in water, since the contributions of each seed 
can be combined to give the dose at a given point. This method provides standardized dosimetry and treatment planning between institutions and does not require additional measurement or $\mathrm{MC}$ dose calculations for specific seed arrangements. The one-dimensional approach has the additional advantage of being independent of seed orientation (e.g., for calculations relying on images where determining the seed's longitudinal axis orientation is difficult), although the two dimensional approximation provides more accurate dose rates, especially for regions close to the sources. ${ }^{10}$

The TG-43 protocol has several disadvantages, namely that it does not account for interseed effects (i.e., interactions of photons emitted from a seed with other seeds in its vicinity) or the presence of heterogeneous media (which may not be waterequivalent) in the treatment region. The validity of ignoring media heterogeneity has been questioned since photon interactions at the low energies relevant to brachytherapy are dominated by the photoelectric effect, which has a cross section for interaction per atom proportional to $\frac{Z^{4.8}}{(h \nu)^{3}}$ in tissue-like media (where $Z$ is the atomic number of the medium and $h \nu$ is the photon energy). ${ }^{11}$ As a result of this strong dependence on $Z$, variations in tissue compositions which change the $Z_{\text {eff }}$ (effective atomic number) of the medium can considerably affect the probability of photon interaction. Nearly all photon energy is absorbed in photoelectric interactions in tissue-like media; thus, both photon attenuation and dose are affected by changes in medium composition. In treatments where high- $Z$ media are introduced, such as in eye plaque brachytherapy, the presence of the high- $Z$ media can also have an effect on dose by reducing the amount of backscatter into areas of interest and by changing the spectrum of fluorescence photons emitted in the high- $Z$ medium region.

Studies with model-based dose calculations have shown that the effects on dose of heterogeneous media and interseed attenuation are significant. ${ }^{6}$ For instance, for prostate treatments, Carrier et al. found that using MC techniques to model heteroge- 
neous tissues and interseed attenuation (based on post-implant patient CT data), the minimum dose received by $90 \%$ of the treatment volume $\left(\mathrm{D}_{90}\right)$ was reduced by a mean of $7 \%$ compared with the TG-43 calculated dose. ${ }^{12}$ Using the MC code BrachyDose, Taylor found that the dose for the Xoft electronic miniature x-ray source was $18 \%$ higher in breast tissue than in water $1 \mathrm{~cm}$ from the source and $1 \%$ lower than in water $6 \mathrm{~cm}$ from the source. ${ }^{13}$ Furthermore, Sutherland et al. ${ }^{14}$ reported that modeling lung tissue and interseed attenuation increased $\mathrm{D}_{90}$ compared to the $\mathrm{D}_{90}$ calculated using the TG-43 approach by up to $36 \%$. Despite the considerable differences found between brachytherapy dose calculations in water and in heterogeneous tissue, research on the dosimetric effects of heterogeneous tissues for ocular (eye plaque) brachytherapy is limited, as will be discussed in the following section.

\subsection{Eye plaque brachytherapy}

Eye plaque brachytherapy is a form of treatment for uveal melanoma (i.e., melanoma of the choroid, iris or ciliary body), the most common form of ocular melanoma which affects roughly 2500 North Americans annually. ${ }^{15}$ It involves the use of brachytherapy sources, which can be either photon-emitting, such as ${ }^{198} \mathrm{Au}$ (Ref. 16), ${ }^{125} \mathrm{I},{ }^{103} \mathrm{Pd}$ and ${ }^{131} \mathrm{Cs}$ (Ref. 17), or beta-emitting, such as ${ }^{106} \mathrm{Ru}$ (Ref. 18,19). When brachytherapy seeds are used, as is the case for photon-emitting sources, the seeds are affixed to a plaque backing with or without an insert; the plaque is then surgically implanted on the surface of the eye next to the tumor region and removed after several days, upon completion of treatment. ${ }^{20}$

Historically, uveal melanoma was treated by enucleation (removal of the eye); however, several alternate treatment options are now available, including external beam radiotherapies (with photons or protons) and eye plaque brachytherapy. Cur- 
rently, eye plaque brachytherapy and enucleation are the most common choices for treatment. ${ }^{15}$ Although not all tumors are eligible for eye plaque brachytherapy, ${ }^{20}$ it has the benefit of superior vision retention and cosmetic results. Furthermore, survival rates have been found to be comparable with those for enucleation. ${ }^{21,22}$

The Collaborative Ocular Melanoma Study (COMS) ${ }^{23}$ recommended the use of standardized COMS plaque consisting of a gold alloy (Modulay) spherical shell backing with a collimating lip and a silicone polymer (Silastic) insert containing ${ }^{125}$ I seeds. However, various plaque designs have been developed and are used with different radionuclides at institutions worldwide. Plaques can have backings made of various gold alloys, ${ }^{24-26}$ stainless steel ${ }^{27,28}$ or acrylic ${ }^{16}$ rather than Modulay. Rather than employing a Silastic seed carrier, plaques can have seeds glued directly to the backings $^{29}$ (sometimes using a very thin Modulay seed guide to position the seeds ${ }^{30}$ ), or may use a thin acrylic layer ${ }^{31}$ or a full (i.e., conforming with the outer surface of the sclera) acrylic insert to hold the seeds in place. ${ }^{27}$ Some institutions use plaques without a collimating lip or a shorter lip than for the COMS plaque. ${ }^{25,26}$ Slotted plaques have also been developed in which seeds are placed in slots within the backing to collimate the radiation ${ }^{32,33}$ in an attempt to reduce scleral dose while retaining dose conformity within the tumor. As tumors can develop in regions where a spherical shell plaque is unsuitable, such as the iris or at the posterior of the eye near the optic nerve, modified plaques for the iris ${ }^{34,35}$ and notched plaques for the optic nerve are used. ${ }^{36}$

Different radionuclides are used for eye plaque brachytherapy based on factors such as the average photon energies emitted (those with smaller average photon energies have steeper dose fall-offs, lowering dose far from the plaque, i.e., outside the tumor volume), and half-life (shorter half-lives are preferred due to radiologic waste concerns ${ }^{17}$ ). In most cases a single radionuclide is used within the plaque, although the use of multiple radionuclides in a plaque has been investigated to optimize the 
dose distribution. ${ }^{37}$ The number of seeds and their distribution within the plaque vary with institution and are dependent on the plaque size used.

As for other areas of brachytherapy, dosimetry practices for ocular brachytherapy remain rudimentary despite its widespread use. ${ }^{38}$ Clinical dose calculations are generally carried out with the patient treated as water-equivalent (i.e., using a TG43 approach $^{9}$ ) and a dose of 85 Gy prescribed to the tumor apex. ${ }^{20}$ This approach involves considerable approximations: in addition to ignoring interseed attenuation and ocular media within the eye, the effects from the presence of the plaque backing and insert are neglected.

As mentioned in section 1.1, the effect of media heterogeneity on dose, including the effect of both the plaque and ocular media, has not been studied in detail for ocular brachytherapy. Some researchers have modeled simplified eye geometry for ocular brachytherapy assuming water media, ${ }^{39}$ or have modeled eye geometry and partial composition for external beam therapy calculations ${ }^{40,41}$ and other applications. $^{42}$ Specifically, the eye model used by Perrata ${ }^{42}$ includes mass densities and conductivities of ocular structures but does not include their elemental compositions. The eye model used by Behrens et al. ${ }^{40,41}$ includes elemental compositions for certain ocular media; however, this model has no iris or tumor, the cornea and sclera are assigned the same composition, and the vitreous and aqueous humors are assumed water-equivalent. Preliminary work studying the presence of the air interface at the front of the eye ${ }^{43}$ and bone surrounding the eye found these to affect dose ${ }^{44}$ when a water-equivalent eye was assumed.

Some groups have studied the effects of various plaque models on dose in water; these effects can vary significantly as a result of the compositional and structural differences between plaque models. The effects of the standard COMS plaque on the dose in water have been widely studied, ${ }^{38,43-46}$ e.g., fully loaded 10-22 mm diameter 
COMS plaques reduce the dose in water $5 \mathrm{~mm}$ from the surface of the insert along the plaque's central axis by an average of $19 \%, 11 \%$ and $9 \%$ for ${ }^{103} \mathrm{Pd},{ }^{125} \mathrm{I}$, and ${ }^{131} \mathrm{Cs}$, respectively. ${ }^{46}$ However, dosimetric plaque effects in water have been characterized for few other plaque models. Granero et al. reported that for the ROPES eye plaque (stainless steel backing and acrylic insert), the stainless steel backing causes a $4 \%$ dose reduction for the plaque fully loaded with ${ }^{125} \mathrm{I}$ seeds in water compared with the same seeds alone in water $;^{28}$ they found the acrylic insert to have a negligible effect on dose. Astrahan et al. reported doses to the whole eye to be lower (while maintaining the prescription dose to the tumor apex) with their slotted plaque in comparison with a standard COMS plaque. ${ }^{32}$ For another plaque model developed by Astrahan et al. which contains a reusable thin seed guide (without a Silastic insert), doses using ${ }^{125} \mathrm{I}$ were $10 \%$ lower at the outer sclera next to the plaque and $11 \%$ higher at the far side of the eye from the plaque than for the COMS plaque (when dose was prescribed to the tumor apex, $5.0 \mathrm{~mm}$ from the inner sclera). ${ }^{30}$ For a similar plaque model, the OSU-NAG plaque (Modulay backing with seeds glued directly to the plaque backing), Zhang et al. reported $10 \%$ and $12 \%$ higher doses than for the COMS plaque at the inner scleral edge closest to the plaque for ${ }^{125} \mathrm{I}$ and ${ }^{131} \mathrm{Cs}$, respectively, and $6 \%$ (for ${ }^{125} \mathrm{I}$ ) and $3 \%$ (for ${ }^{131} \mathrm{Cs}$ ) lower doses at the retina on the opposite side of the eye. ${ }^{29}$ Puusaari et al. compared the calculated doses for a slotted plaque design to those for the plaques used at their institution in Helsinki, Finland (gold backings with Silastic insert and no collimating lip) and found the median doses to the optic nerve and macula were lower by a mean of $45 \%$ to $38 \%$ (for a variety of tumor sizes), respectively, for the slotted plaque design. ${ }^{33}$

Given that eye plaque brachytherapy does not always achieve local tumor control and metastases or secondary effects such as retinopathy, optical neuropathy and cataracts $^{47}$ may develop, accurate dosimetry for eye plaque brachytherapy is essen- 
tial. Despite research aimed at improving ocular brachytherapy dosimetry (see e.g., Thomson et al., ${ }^{44}$ Rivard et al. ${ }^{48}$ and Chiu-Tsao et $\left.a .^{38}\right)$, the patient continues to be treated as water-equivalent. With the development of techniques for advanced dose calculations for brachytherapy, full model-based dose calculations for ocular brachytherapy should be possible; ${ }^{6}$ however, these require an anatomically and compositionally realistic eye model, as well as representative models of the eye plaques currently available in the clinic.

\subsection{BrachyDose}

Dose calculations for brachytherapy, whether for TG-43 parameterization or for modelbased dose calculations, often use Monte Carlo techniques to simulate radiation transport and energy deposition. Various Monte Carlo codes exist (e.g., MCNP49,50 and GEANT4 ${ }^{51}$ ), including BrachyDose, ${ }^{52,53}$ an EGSnrc ${ }^{54}$ user-code for brachytherapy applications.

BrachyDose has the ability to model radiation transport and energy deposition in rectilinear, cylindrical, conical, and spherical geometries, and any combination thereof through its use of Yegin's multi-geometry package. ${ }^{55}$ Media assignments in each of these geometries are customizable, enabling precise modeling of sources and other geometric objects, such as eye plaques. Irregular geometries can also be modeled using a rectilinear voxelized phantom (where a voxel is a 3-dimensional pixel) with each voxel assigned a specific medium/composition; this allows dose calculations for realistic treatment geometries (including tissue geometry and composition) to be readily implemented in Brachydose. Voxel boundaries and thus model resolution in BrachyDose are also customizable.

BrachyDose approximates the dose by calculating the collision kerma (kinetic 
energy released per unit mass) to each region or voxel. This approximation is valid since electron ranges at these energies are submillimeter in length, and bremsstrahlung radiation is highly unlikely; ${ }^{11}$ it is therefore unnecessary to model electron transport. The collision kerma, which represents the energy transferred by a photon to charged particles that is ultimately absorbed via ionizational interactions, is calculated using the following equation:

$$
K_{c o l}=\sum_{m} \Phi_{m} E_{m}\left(\frac{\mu_{e n}}{\rho}\right)_{m}
$$

where $\Phi_{m}$ is the photon fluence (number of photons per unit area) and $\left(\frac{\mu_{e n}}{\rho}\right)_{m}$ is the mass energy absorption coefficient in the medium corresponding to the photon energy $E_{m}$. BrachyDose uses a tracklength estimator to calculate the collision kerma to a voxel as follows:

$$
D^{j}=K_{c o l}^{j}=\frac{1}{V_{j}} \sum_{i} E_{i} t_{i}\left(\frac{\mu_{e n}}{\rho}\right)_{i}
$$

where $D^{j}$ and $K_{c o l}^{j}$ are the dose and collision kerma to the $j^{\text {th }}$ voxel, respectively, $V_{j}$ is the volume of the $j^{\text {th }}$ voxel,,$t_{i}$ is the tracklength of the $i^{\text {th }}$ photon through the voxel, and $\left(\frac{\mu_{e n}}{\rho}\right)_{i}$ is the mass energy absorption coefficient for the medium in the voxel corresponding to the energy of the $i^{\text {th }}$ photon.

Although it is generally unnecessary, BrachyDose has the capability of modeling electron transport, as both photon and electron transport cut-offs are defined by the user. The user is provided further flexibility with the choice of applying volume corrections to voxels which are intersected by sources (or to set the dose in these voxels to zero) and of recycling particles (where for multiple seed simulations, the photons escaping one seed are re-used at each seed location). It is also possible to score the 
fluence spectrum in a voxel and the energy-weighted spectrum at the source surface, and to separate dose to voxels which result from primary and scattered photons. Interseed interactions can be included or ignored, and the type of photon and electron interactions to be modeled can be specified (i.e., bound Compton scattering, Rayleigh scattering, atomic relaxations, and electron impact ionization).

BrachyDose has been benchmarked through various comparisons with calculations using different Monte Carlo codes or experimental measurements published by other authors. For example, primary and scatter dose calculations in BrachyDose have been benchmarked by Taylor and Rogers ${ }^{56}$ against analogous calculations in other

published work. ${ }^{57-59}$ Source models used in BrachyDose have been validated through the general agreement of TG-43 parameters calculated using BrachyDose ${ }^{52,56,60,61}$ to published data. Photon energy spectra calculated with BrachyDose for the Xoft Axxent x-ray source ${ }^{13,62}$ have also been found to agree with measurements made by Rivard et al. ${ }^{63}$ Further, dose distributions calculated for the standardized COMS plaque using BrachyDose ${ }^{44}$ and $\mathrm{MCNP}^{46}$ have shown good agreement.

\subsection{Thesis Outline}

Doses calculated using the TG-43 protocol are considerably different than those using model-based dose calculations due to the low photon energies used in brachytherapy. The sensitivity of dose to media heterogeneities, however, has not been widely investigated for ocular brachytherapy. Ocular media have not been accurately modeled, as eye models presented in the literature assume the eye to be radiologically equivalent to water or include the elemental compositions and dimensions of few ocular structures. Therefore, comparisons of doses to ocular structures have not been performed in the context of ocular media. The presence of the eye plaque has been shown to 
affect dose, however the few studies which have investigated these effects have generally assumed a radiologically water-equivalent eye and are difficult to compare due to the varying plaque sizes, seed arrangements within the plaques, and methods used to calculate or measure dose.

This work aims to contribute to the development of a comprehensive understanding of dosimetry for ocular brachytherapy, including a thorough understanding of ocular media effects and plaque effects on dose for three photon-emitting radionuclides commonly used in treatment: ${ }^{125} \mathrm{I},{ }^{103} \mathrm{Pd}$, and ${ }^{131} \mathrm{Cs}$. This requires both the development of an anatomically and compositionally realistic human eye model, as well as the development of representative plaque models to cover the range of plaques currently available for clinical use.

This work is presented in two parts. The first part, in Chapter 2, describes the author's development of a full eye model including geometry and composition for the eye and surrounding tissues. It includes a detailed analysis of the effects of the ocular media and surrounding media (rather than water) on dose to volumes (i.e., maximum, minimum and average doses to ocular structures) and points of interest within the eye for the widely-used $16 \mathrm{~mm}$ COMS plaque. Chapter 2 also investigates the sensitivity of the full eye model by comparing doses for the plaque in the full eye model to those with an alternate tumor composition and position, and by varying the choice of media surrounding the eye (including investigating the dosimetric effects of the air interface at the front of the eye). The second part of this work, in Chapter 3 , describes the author's development of five representative plaque models and compares their dosimetric effects in water and in the full eye model to those for the widely-used, previously modeled, COMS plaque. The Monte Carlo simulation parameters used to calculate dose, as well as the sources used in the simulations and the dose notation used in both parts of this work (Chapters 2 and 3) are described in the Methods 
section of Chapter 2. Chapter 4 summarizes all results presented in this study, and provides an outlook on future work in the field of ocular brachytherapy dosimetry.

The development of the eye model presented in this thesis enables the novel comparison of doses to ocular structures using ocular media compositions to those using a water-equivalent eye for a range of photon-emitting brachytherapy sources. It also provides comparison between the effects of different types of plaques in both an eye model and in water using a consistent seed arrangement, plaque size, and dose calculation method, which has not previously been done. This study aims to improve general understanding of eye plaque brachytherapy dosimetry, working towards the use of accurate model-based dose calculations to achieve better patient treatment outcomes. 


\section{Chapter 2}

\section{Development of an eye model and investi- gation of its effect on dose for the COMS plaque}

\section{$2.1 \quad$ Introduction}

In this chapter, a geometrically and compositionally realistic three-dimensional rectilinear voxelized model of the eye and surrounding tissues is developed. Using Monte Carlo dose calculations, the effects of ocular media and surrounding tissues on radiation transport and energy deposition are investigated, focusing on the widely-used COMS plaque. Three main simulation geometries are modeled: the brachytherapy seeds in water without interseed effects (i.e., a TG-43 simulation), the fully loaded COMS plaque in a water phantom, and the fully loaded COMS plaque in the full eye model. Simulations that replace the tumor with alternate media assignments and that replace the surrounding tissues with water, with and without an air interface, are also performed. As the anatomically realistic eye model permits investigation of doses to volumes within structures of interest, in addition to the current method of reporting dose to points (in water), maximum, minimum and average doses to various ocular structures are investigated and compared to doses at points of interest for the three radionuclides modeled $\left({ }^{125} \mathrm{I},{ }^{103} \mathrm{Pd}\right.$, and $\left.{ }^{131} \mathrm{Cs}\right)$. 


\subsection{Methods}

This chapter is comprised of two parts: the development of a representative computational model of the human eye (section 2.2.1), and Monte Carlo simulations that make use of the representative eye model and other simulation geometries (section 2.2.2).

\subsubsection{Development of a human eye model}

Ocular geometry and composition vary within the population; the eye model developed in this chapter is representative of an adult eye and its surrounding tissues. The dimensions and shapes of ocular structures selected for the model are based on published literature, as are the elemental compositions and densities for ocular media. Tissues surrounding the eye are modeled using a reference computational phantom from the International Commission on Radiological Protection (ICRP).

\section{Dimensions and shapes of ocular structures}

The computational model is developed for a right eye. The coordinate system used in the current work, shown in Fig. 2.1(a), has the eye centered at the origin, with the $y$ axis oriented vertically upwards, the $x$ axis pointed horizontally towards the anterior of the body (through the center of the lens and iris), and the $z$ axis pointed horizontally towards the right hand side.

Bounding surfaces can be used to define the extent of most ocular structures due to their spherical or cylindrical symmetry and are employed to create the rectilinear voxelized eye model. As the dimensions and composition of ocular structures vary between individuals due to age, sex, existing eye conditions (e.g., myopia, hyperopia), 


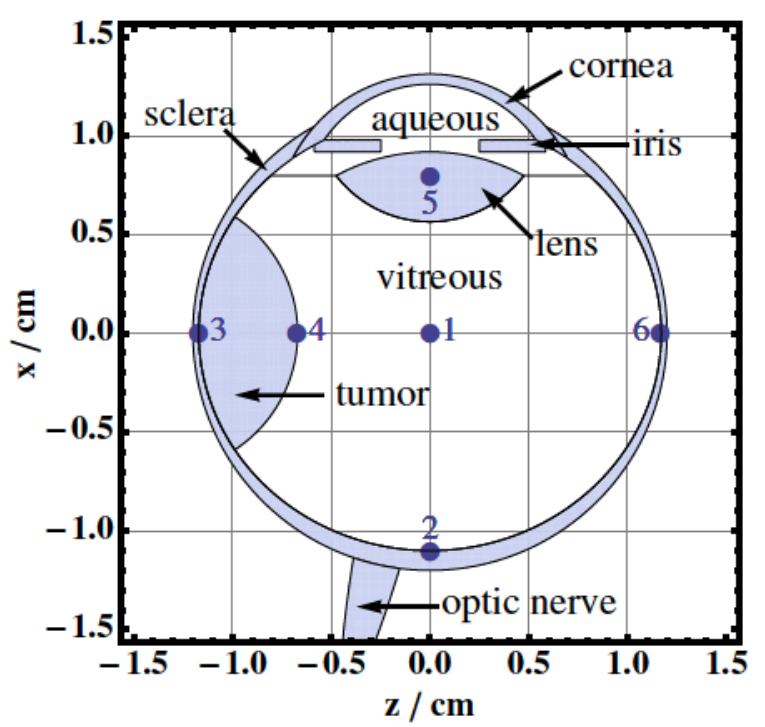

(a)

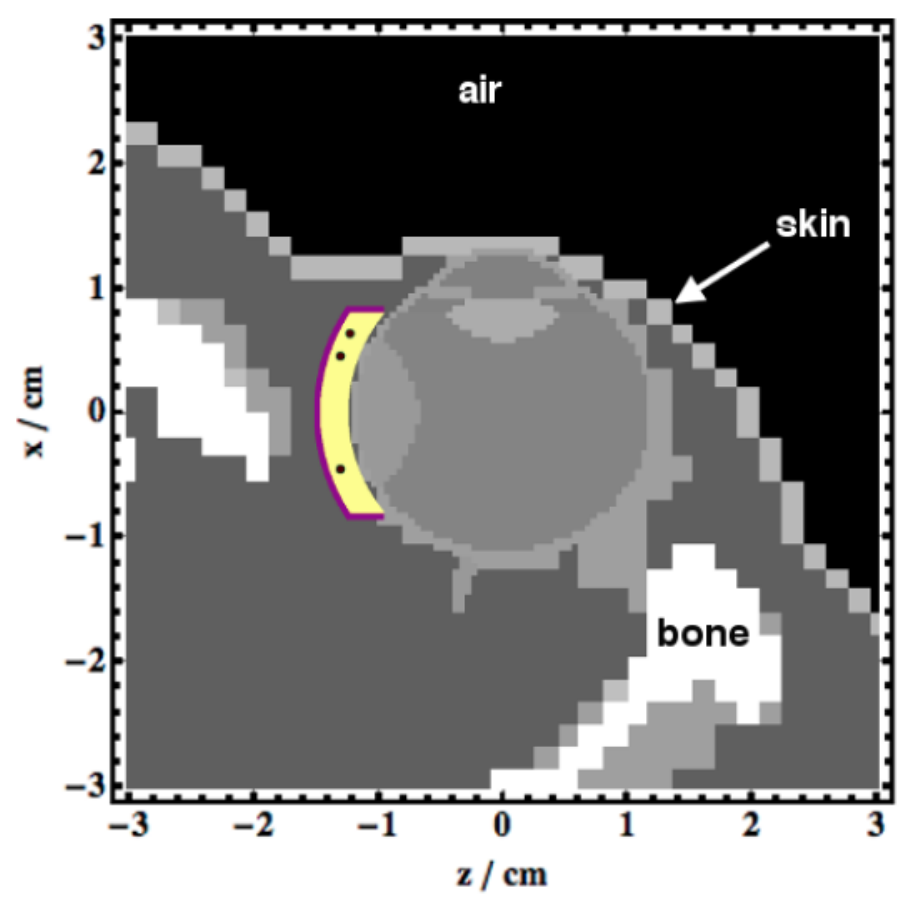

(b)

Figure 2.1: Visualization of (a) bounding surfaces for the right eye model with ocular structures indicated and points of interest as follows: 1 . eye center, 2 . fovea, 3. near side of sclera, 4. tumor apex, 5. lens center and 6. far side of sclera; and (b) plaque and seeds with the voxelized right eye model surrounded by media from ICRP reference computational phantom (i.e., the full eye model). In both figures, the $y$ axis is oriented vertically upwards, the $x$ axis is directed towards the anterior of the body, and the $z$ axis points laterally to the right. The central $(y=0 \mathrm{~cm})$ slice in the $x z$ plane is shown in both (a) and (b); for (b) the voxel centers in this slice lie at $y=0.025 \mathrm{~cm}$ as the voxel boundaries lie at $y=0$ $\mathrm{cm}$. 
etc., the bounding surfaces are based on average values found within the literature.

For the eye model, the outer bounding surface of the sclera is a sphere $24 \mathrm{~mm}$ in diameter, ${ }^{64-66}$ scleral diameter generally ranges from $21-26 \mathrm{~mm} .{ }^{64}$ The inner sclera is defined by an ellipsoid such that scleral thickness is $0.3 \mathrm{~mm}$ in the plane separating the anterior and posterior halves of the eye and $1 \mathrm{~mm}$ at the anterior and posterior poles of the eye. ${ }^{64}$ The cornea is bounded by two spheres with radii $7.8 \mathrm{~mm}$ and 6.5 $\mathrm{mm}$, respectively. ${ }^{64}$ The larger sphere (i.e., the outer cornea) is positioned such that its intersection with the outer sclera forms a circle of diameter $11.7 \mathrm{~mm},{ }^{64}$ and the smaller sphere, the inner cornea, is placed so that the corneal thickness is $0.52 \mathrm{~mm}$ on the $x$ axis. ${ }^{64}$ The iris is bounded by two cylinders with diameters of $5 \mathrm{~mm}$ and $11.7 \mathrm{~mm}^{65}$ and two planes such that it has a thickness of $0.6 \mathrm{~mm}$ in the $x$ direction and its anterior surface lies at the intersection of inner sclera and cornea. The lens volume is defined by two spheres with radii of $10 \mathrm{~mm}$ and $6 \mathrm{~mm}$ for the anterior and posterior surfaces, respectively; their intersection creates a lens diameter of $9.5 \mathrm{~mm}$ at its widest point in the $y z$ plane; $;{ }^{64}$ these values can range from 8-14 mm, ${ }^{64} 4.5-7.5$ $\mathrm{mm}^{64}$ and 9-10 $\mathrm{mm},{ }^{65}$ respectively.

The region bounded by the inner cornea and the anterior curved portion of the lens is the aqueous humor, or aqueous, and the remaining region enclosed by the inner sclera is the vitreous humor, or vitreous. The optic nerve is also included, although its intersection with the inner sclera, the optic disk, is not visible in Fig. 2.1 since it lies below the $x z$ plane at $y=-1 \mathrm{~mm}$ and $z=-3 \mathrm{~mm} .{ }^{64}$ The optic nerve volume is defined by three different cylindrical segments with a total length of roughly $1.1 \mathrm{~cm}$ increasing in diameter from $1.5 \mathrm{~mm}$ at the optic disk, to $2.5 \mathrm{~mm}$ mid-sclera, and finally to 3.5 $\mathrm{mm}$ at the outer sclera. ${ }^{64}$ The optic nerve's total length is not modeled as doses to points on the optic nerve far from the plaque are very low. Other structures, such as the ciliary body, are not included in the eye model due to either their geometric 
complexity or dimensions smaller than the chosen resolution.

A medium-sized tumor is modeled in the region of the choroid, a thin vascular layer of the eye attached to the inner sclera which is not included in the eye model. The tumor is modeled as a segment of a sphere to emulate the most common domeshaped choroidal uveal melanoma. ${ }^{15}$ The tumor has a diameter of $12 \mathrm{~mm}$ in the $y$ direction at its intersection with the inner sclera and its apex lies $5 \mathrm{~mm}$ inward from the inner edge of the sclera. For most of the current work, the tumor is chosen to lie on the nasal (medial) side of the right eye, centered on the $z$ axis, although choroidal melanoma commonly occurs in other orientations. The dose distributions for two other tumor positions, one at the posterior of the eye and the other centered along the $z$ axis on the lateral side, are therefore also investigated in this chapter in a subset of calculations. The same diameter and apex to inner sclera distance is used for all tumor locations.

\section{Elemental compositions and densities of ocular media}

The compositions of the various eye structures are not all available within the scientific literature; further, these vary between individuals. Elemental compositions and densities used in the current study for all ocular structures in the eye model are listed in Table 2.1. These data are either directly from the published literature (for the vitreous, ${ }^{64,67}$ aqueous ${ }^{64,68}$ and lens ${ }^{69}$ ) or are derived as described below using information from the published literature. Mass energy absorption and attenuation coefficients for all media are generated using the EGSnrc user-codes g and examin, ${ }^{54}$ with photon cross sections taken from the XCOM database. ${ }^{70}$ 


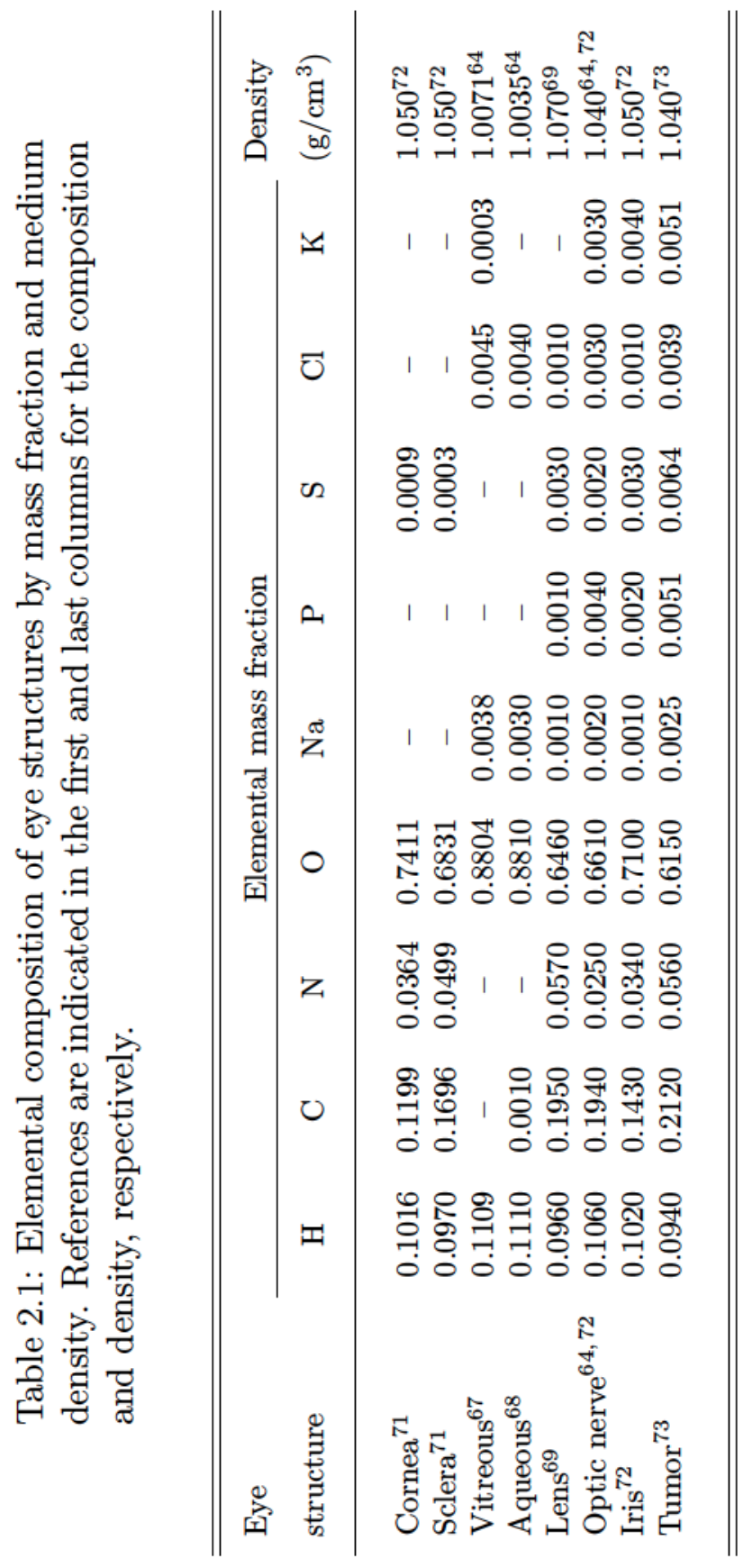


General scleral and corneal compositions are taken from $\mathrm{Meek}^{71}$ where scleral composition is given as $28.8 \%$ collagen, $0.64 \%$ elastin, $<3 \%$ other proteins, $<1 \%$ proteoglycans and $68 \%$ water by mass and corneal composition is given as $14.6 \%$ collagen, $8.2 \%$ other proteins, $1 \%$ proteoglycans and $76.2 \%$ water by mass. The collagen used for the eye model is assumed to be composed entirely of the most common tripeptide sequence found in collagen. ${ }^{74}$ All other non-water media listed in these general compositions are proteins and are assigned the general protein composition given in Zierold et al. ${ }^{75}$ Mass energy absorption coefficient ratios of the sclera and cornea to water are relatively insensitive to the protein composition for collagen and the other non-water media they contain: using the protein composition from International Commission on Radiological Protection (ICRP) Report $89^{76}$ rather than the composition from Zierold et al. ${ }^{75}$ for the corneal and scleral proteins other than collagen changed mass energy absorption coefficient ratios by less than $1 \%$ for the cornea and $0.2 \%$ for the sclera for photon energies below $50 \mathrm{keV}$. Substituting the collagen composition with the ICRP protein composition resulted in changes in mass energy absorption coefficient ratios to water of up to $1 \%$ and $3 \%$ for the cornea and sclera, respectively. Densities for these two structures are not found within the literature and are thus assigned a density of $1.05 \mathrm{~g} / \mathrm{cm}^{3}$, the density of "muscle skeletal 2" in Woodard and White. ${ }^{72}$

Due to a lack of data within the literature, the composition and density of the optic nerve is assumed to be that of "brain white matter" in Woodard and White ${ }^{72}$ based on its organizational similarity to this structure, ${ }^{64}$ whereas the iris is assumed to be "muscle skeletal 2" in Woodard and White ${ }^{72}$ since it is comprised predominantly of muscle. ${ }^{77}$

The composition and density of "melanoma 1" of Maughan et al..$^{73}$ is assigned to the tumor. Using the composition of Maughan et al. for "melanoma 2" results in 
mass energy absorption coefficient ratios of melanoma to water up to $6 \%$ higher than for "melanoma 1"; thus, the more radiologically water-like composition of "melanoma 1 " is used. The elemental composition of uveal melanoma may differ from the compositions of other melanomas, however, the elemental composition of uveal melanoma is not currently available. Further, as with all tissues, variation in elemental composition likely exists within the population.

\section{Model of the eye and surrounding tissues}

A voxelized eye model with rectilinear voxels is created using the bounding surfaces described: each $(0.5 \mathrm{~mm})^{3}$ voxel is assigned the composition of a given structure if its center lies within the structure's bounding surfaces (Fig. 2.1, page 15).

Media surrounding the eye are represented using the voxelized ICRP Female Reference computational phantom. ${ }^{3}$ The female phantom is chosen rather than the male phantom due to its superior resolution of $4.84 \mathrm{~mm}$ vertically and $1.775 \mathrm{~mm}$ horizontally in both directions. Media defined in the ICRP phantom are based on segmentation of a high-resolution full-body computed tomography scan of a female and are modified (i.e., voxel scaling, addition of voxels containing adipose) to correspond with the geometry and composition of the Reference Female. ${ }^{76}$ Elemental compositions for the media assigned in this phantom are described in the ICRP Report $110 .^{3}$

The voxelized eye model is superimposed on the segmented eye region in the ICRP computational phantom by aligning the eyes' geometric centers. The combined model of the eye plus surrounding tissues from the computational phantom is output in the widely-used egsphant format ${ }^{78}$ and has a $0.5 \mathrm{~mm}$ resolution for the $3 \mathrm{~cm}$ wide cubic region centered on the eye and the same resolution as the ICRP phantom for 
areas outside this region. A $5 \mathrm{~cm}$ margin of the ICRP phantom data is included around the eye in each direction.

The chosen size for the eye model coincides well with the eye region in the ICRP phantom (Fig. 2.1(b)), although there is some extension of ICRP phantom eye beyond the detailed eye model. A single voxel layer of skin also covers part of the front of the eye, representing a partially closed eye.

\subsubsection{Monte Carlo simulations}

Photon transport cut-offs are set to $1 \mathrm{keV}$. All electrons are assumed to deposit their energy locally as the range of electrons relevant to the sources studied is much smaller than the minimum voxel size of $0.5 \mathrm{~mm}$ (at $30 \mathrm{keV}$, electron CSDA range in water is on the order of $0.02 \mathrm{~mm}$ and for lower energies it is shorter $\left.{ }^{11}\right)$. Bound Compton scattering, Rayleigh scattering and atomic relaxations are all modeled.

BrachyDose makes use of Yegin's multi-geometry package ${ }^{55}$ to fully model the eye plaque and brachytherapy sources. Unlike the eye model, the eye plaque and sources are therefore not represented by rectilinear voxels. In this chapter, the eye plaque selected for simulations is the widely-used standardized $16 \mathrm{~mm}$ COMS plaque ${ }^{44}$ which contains 13 sources and is suitable for treatment of a tumor $12 \mathrm{~mm}$ in diameter (measured from one end of the tumor's intersection with the sclera to the other). ${ }^{23}$ The plaque consists of a gold alloy (Modulay) backing and a silicone-polymer (Silastic) seed carrier attached to the concave surface of the backing. ${ }^{44}$

Three different radionuclides are studied, ${ }^{125} \mathrm{I},{ }^{131} \mathrm{Cs}$ and ${ }^{103} \mathrm{Pd}$, with spectra taken from TG-43U1 $1^{10}$ for ${ }^{125} \mathrm{I}$ and ${ }^{103} \mathrm{Pd}$, and from Rivard et al. ${ }^{79}$ for ${ }^{131} \mathrm{Cs}$. The seed models are the GE Healthcare/Oncura model 6711 for ${ }^{125} \mathrm{I}$, the IsoRay Medical model CS-1 Rev2 for ${ }^{131}$ Cs and the Theragenics, Theraseed model 200 for ${ }^{103} \mathrm{Pd}$; these seeds 
have average emitted photon energies of $27.3 \mathrm{keV}, 30.3 \mathrm{keV}$ and $20.9 \mathrm{keV}$, respectively, and half-lives of 59.4 days, 9.69 days and 17.0 days, respectively. ${ }^{80}$ Details regarding the ${ }^{125} \mathrm{I}$ and ${ }^{103} \mathrm{Pd}$ seeds are available in the Carleton Laboratory for Radiotherapy Physics TG-43 Parameter Database $;{ }^{81}$ the ${ }^{131}$ Cs model dimensions are from the work of Rivard. ${ }^{79}$

In the full eye model, the eye plaque containing the brachytherapy seeds is placed in contact with the outer sclera adjacent to the tumor; the plaque's center is aligned with the central tumor axis. The COMS plaque has an inner radius of curvature of $12.3 \mathrm{~mm}$ (defined by the Silastic insert) and, as such, approximately conforms with the outer edge of the sclera against which it is placed; the maximum distance between the outer sclera and the Silastic insert is $0.5 \mathrm{~mm}$. Hence, for most simulations, the plaque is centered on the $z$ axis on the nasal side of the eye in conjunction with the tumor location (Fig. 2.1(b)).

In addition to simulations of the eye plaque in the representative model of the eye plus surrounding tissues (i.e., the full eye model), various other simulations are performed. To investigate the effects of ocular media, the full Modulay/Silastic plaque (containing the thirteen seeds) is modeled in a $62 \times 62 \times 62 \mathrm{~cm}^{3}$ homogeneous water phantom. Simulations of the sources in a homogeneous water phantom with no plaque and no interseed attenuation, which approximate calculations performed under the TG-43 protocol, are also carried out (with doses denoted $\mathrm{D}_{T G-43}$ ) in order to compare doses from the plaque in water (denoted $\mathrm{D}_{w, w}$ ) and in our full eye model (denoted $\left.\mathrm{D}_{m, m}\right)$ with those from the widely-used TG-43 approach. In general, dose to the local medium in each voxel is computed and reported. In a subset of simulations, dose to water voxels is computed with radiation transport carried out in the full eye model $\left(\mathrm{D}_{w, m}\right)$ in order to isolate the effects of ocular media on radiation transport alone. The notation for the simulation geometries used is summarized in Table 2.2. 
Table 2.2: Summary of simulation geometries and dose notation used in this work.

\begin{tabular}{lc}
\hline \hline Dose notation & Simulation Description \\
\hline $\mathrm{D}_{T G-43}$ & $\begin{array}{c}\text { radiation transport and dose deposition in a water phantom } \\
\text { with seeds alone and no interseed attenuation } \\
\mathrm{D}_{w, w} \\
\text { radiation transport and dose deposition for the eye } \\
\text { plaque within a water phantom }\end{array}$ \\
$\mathrm{D}_{m, m}$ & $\begin{array}{c}\text { radiation transport and dose deposition for the eye plaque } \\
\text { within the full heterogeneous eye and surrounding tissues } \\
\text { (i.e. within the full eye model) }\end{array}$ \\
$\mathrm{D}_{w, m}$ & $\begin{array}{c}\text { radiation transport for the eye plaque within the full heterogeneous } \\
\text { eye and surrounding tissues (i.e. within the full eye model), and } \\
\text { dose deposition scored as if in water }\end{array}$ \\
\hline \hline
\end{tabular}

The sensitivity of doses within the eye to the surrounding media is assessed in a separate set of simulations, where tissues surrounding the eye model (from the ICRP phantom) are replaced with water, both with and without a planar air interface at the front of the eye. Simulations are also performed in which the tumor material is replaced with "melanoma 2" of Maughan et al. ${ }^{73}$ Group 3 male soft tissue from White et al. ${ }^{82}$ and water to investigate the effect of tumor composition on dose.

For each simulation, $10^{10}$ histories are modeled, resulting in statistical $(1 \sigma)$ dose uncertainties in the full eye model of less than $0.1 \%$ at the tumor apex, $0.1-0.2 \%$ at the center of the eye, and $0.3-0.5 \%$ at the opposite side of the sclera to the plaque for all radionuclides studied. Uncertainty on the dose is also affected by the uncertainties on ocular media compositions (investigated in section 2.3.3, page 37) and the cross sections for all media used in the simulations. ${ }^{70}$ To achieve a $2 \%$ statistical uncertainty on the dose at the tumor apex in the full eye model, the total simulation time is 3.6 minutes for ${ }^{125} \mathrm{I}, 2.8$ minutes for ${ }^{103} \mathrm{Pd}$, and 3.4 minutes for ${ }^{131} \mathrm{Cs}$ using a single 3.00 
$\mathrm{GHz}$ Intel Xeon processor. These times should be sufficient for clinical treatment planning, although they do not include contouring of patient geometry which might be necessary if more accurate dose calculations are needed.

\subsection{Results and Discussion}

\subsubsection{Effect of ocular media on dose}

\section{Mass energy absorption and attenuation coefficients}

Mass energy absorption and attenuation coefficient ratios (ocular media to water) are shown in Fig. 2.2 for all eye structures modeled. Significant differences between mass energy absorption and attenuation coefficients for the different ocular media and water are observed in the average emitted photon energy range (between 21 and $30 \mathrm{keV}$ ): mass energy absorption coefficients are higher for ocular media than those for water by up to $10.5 \%$ for the tumor, $7 \%$ for the vitreous, $5.5 \%$ for the aqueous, $3.5 \%$ for the iris and $1.5 \%$ for the optic nerve, and are lower than those for water by as much as $12 \%$ for the sclera, $9.5 \%$ for the lens and $8 \%$ for the cornea. Mass energy absorption and attenuation coefficient ratios for a given ocular medium relative to water generally vary by a few percent over the energy range of 21 to 30 $\mathrm{keV}$ (corresponding to the average photon energies emitted by ${ }^{125} \mathrm{I},{ }^{103} \mathrm{Pd}$ and ${ }^{131} \mathrm{Cs}$ ).

The observed differences in mass energy absorption and attenuation coefficients for the ocular media compared to water may be understood through consideration of the elemental compositions and densities listed in Table 2.1. Compared to water, the presence of elements of higher atomic number in ocular media increases the effective atomic number and hence the photoelectric cross section, resulting in increases in energy absorption. In contrast, the presence of carbon and nitrogen in some ocular 


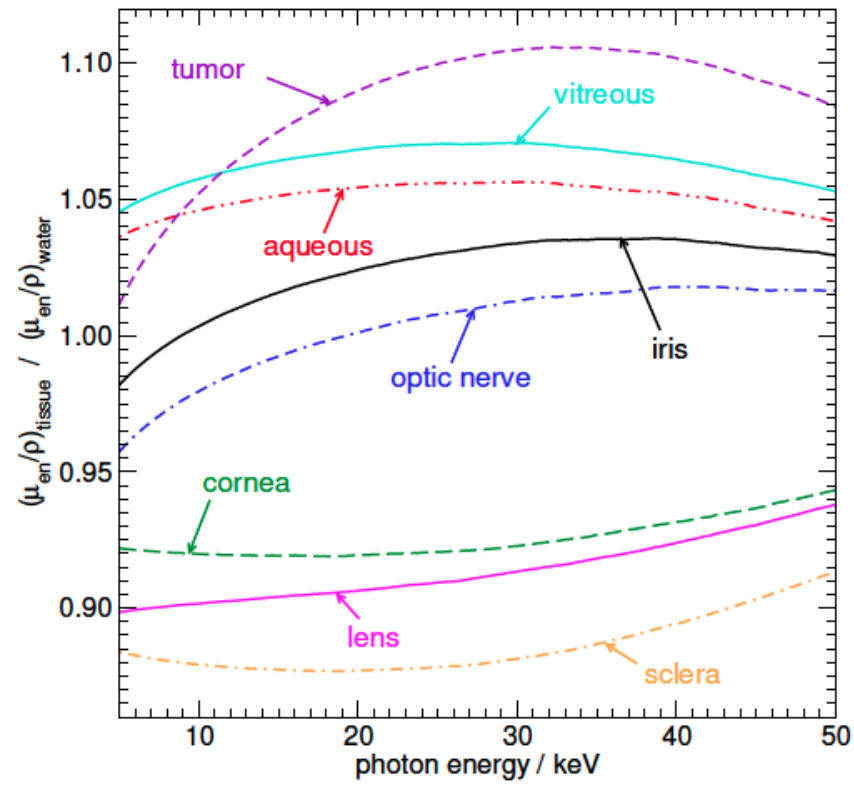

(a)

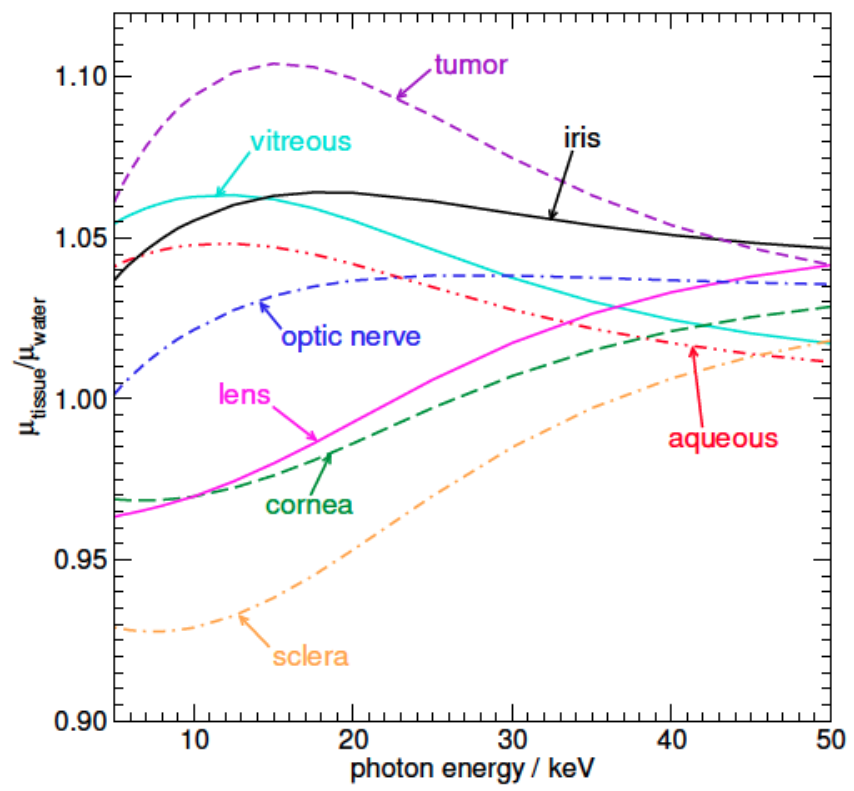

(b)

Figure 2.2: Ratios of (a) mass energy absorption coefficients and (b) attenuation coefficients for tissues used in the eye model to those of water for the $5-50 \mathrm{keV}$ photon energy range. Elemental compositions for ocular media are found in Table 2.1 (page 18). 
media lowers the effective atomic number and decreases the photoelectric cross section. Mass energy absorption and attenuation coefficient ratios of ocular media to water do not vary identically with photon energy due to differences in proportions of coherent and incoherent scattering and photoelectric interactions at different photon energies.

\section{Radiation transport and energy deposition throughout the eye}

Differences in dose distributions from water and full eye model simulations are anticipated given differences in energy absorption and attenuation in ocular media. This

section focuses on comparing doses for the plaque in the full eye model $\left(\mathrm{D}_{m, m}\right)$ and for radiation transport with the plaque in the full eye model but dose scoring in water $\left(\mathrm{D}_{w, m}\right)$ to doses for the plaque in water $\left(\mathrm{D}_{w, w}\right)$. As dose is scored in water for $\mathrm{D}_{w, m}$, the ratio of $\mathrm{D}_{w, m}$ to $\mathrm{D}_{w, w}$ serves as an indicator of differences in photon fluence due to radiation transport within the eye model rather than water.

For the plaque in the full eye model, the dose $\left(\mathrm{D}_{m, m}\right)$ along the tumor, or $z$, axis (Fig. 2.3(a)) is larger than for the plaque in water $\left(\mathrm{D}_{w, w}\right)$ throughout the tumor region due to the tumor's larger mass energy absorption coefficient than for water. At the tumor base, $\mathrm{D}_{m, m}$ is roughly $10-11 \%$ larger than $\mathrm{D}_{w, w} ; \mathrm{D}_{m, m}$ is nearer $\mathrm{D}_{w, w}$ at the apex due to increased photon attenuation in the tumor material compared with water, confirmed by the decreasing $\mathrm{D}_{w, m}$ to $\mathrm{D}_{w, w}$ ratio in Fig. 2.3(a) through the tumor. The decrease in photon fluence within the tumor is slightly more pronounced for the lower-energy ${ }^{103} \mathrm{Pd}$ source than the other two higher-energy sources, consistent with the relevant attenuation coefficient ratios of tumor medium to water. At the tumor apex, the maximum difference in dose ratios between radionuclides is $4 \%$.

The decreasing $\mathrm{D}_{w, m} / \mathrm{D}_{w, w}$ curve in the vitreous region (Fig. 2.3(a)) suggests 


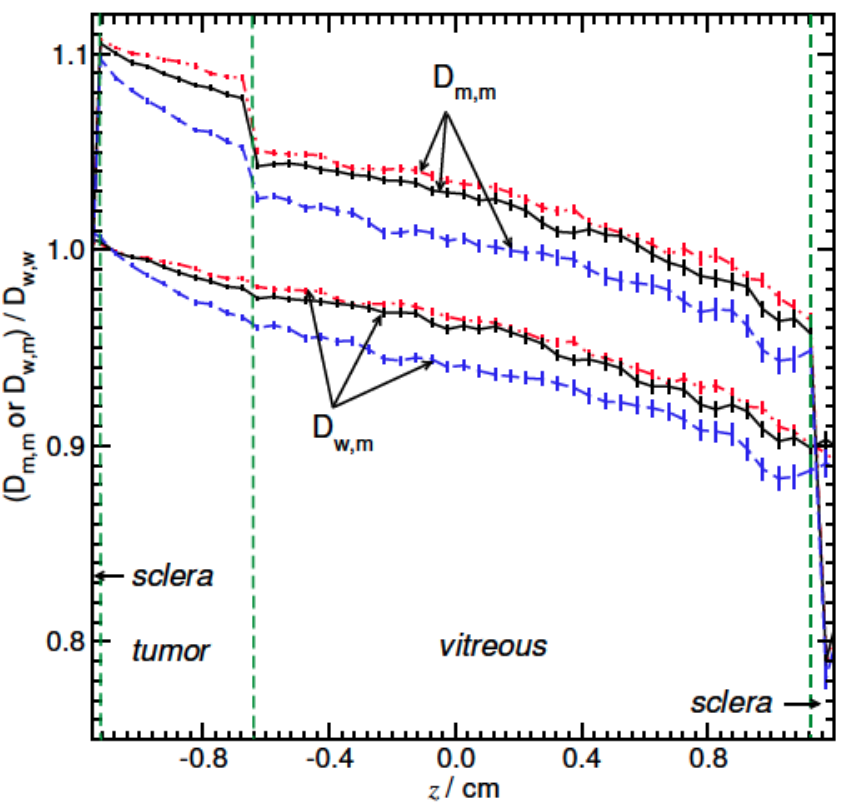

(a)

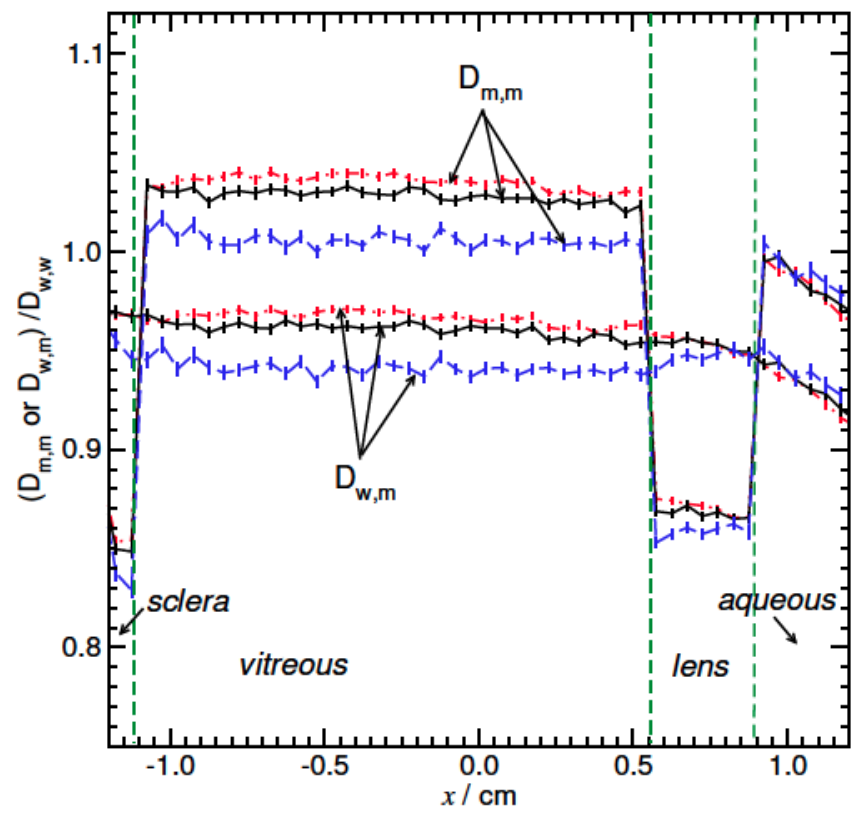

(b)

Figure 2.3: Ratios of dose for the COMS plaque in the full eye model $\left(\mathrm{D}_{m, m}\right)$ or dose to water with radiation transport in the full eye model $\left(\mathrm{D}_{w, m}\right)$ to dose for the plaque in water $\left(\mathrm{D}_{w, w}\right)$ along (a) tumor, or $z$, axis (voxel centers are at $x=0.025 \mathrm{~cm}$ and $y=0.025 \mathrm{~cm}$ as the voxel boundaries are at $x=0 \mathrm{~cm}$ and $y=0$ $\mathrm{cm}$ ) and (b) lens, or $x$, axis (voxel centers are at $y=0.025$ and $z=0.025 \mathrm{~cm}$ as the voxel boundaries are at $y=0 \mathrm{~cm}$ and $z=0 \mathrm{~cm})$. Doses are calculated using the same air kerma strength for all simulation geometries, and the extent of ocular structures are indicated in the figures. Values are represented by solid black lines for ${ }^{125} \mathrm{I}$, by blue dashed lines for ${ }^{103} \mathrm{Pd}$, and by red dash-dotted lines for ${ }^{131} \mathrm{Cs}$. 
that the photon fluence decreases in vitreous compared with the plaque in water simulation. Thus, although $\mathrm{D}_{m, m}$ is larger than $\mathrm{D}_{w, w}$ by $3-5 \%$ in the vitreous near the tumor apex, at the far end of the vitreous $\mathrm{D}_{m, m}$ is lower than $\mathrm{D}_{w, w}$ by $3-6 \%$. As the sclera only consists of one voxel on the $z$ axis, the decreased attenuation in the sclera compared with water does not significantly affect dose to other ocular structures.

Along the lens axis of the eye ( $x$ axis, Fig. 2.3(b)), there is a dose reduction in the lens region for the plaque in the full eye model $\left(\mathrm{D}_{m, m}\right)$ of roughly $13-14 \%$ when compared with the plaque in water $\left(\mathrm{D}_{w, w}\right)$. This is caused by a combination of the lower mass energy absorption coefficient in lens media than in water, and by a lower

photon fluence in the lens region for $\mathrm{D}_{m, m}$ (observed by the lower $\mathrm{D}_{w, m}$ compared with $\mathrm{D}_{w, w}$ in the lens region of Fig. 2.3(b)) resulting from radiation transport through the tumor and vitreous as opposed to water. The decreases in $\mathrm{D}_{m, m} / \mathrm{D}_{w, w}$ and $\mathrm{D}_{w, m} / \mathrm{D}_{w, w}$ with increasing $x$ in the aqueous region (Fig. 2.3(b)) are due to the presence of the air interface at the front of the eye in the full eye model which results in less backscatter to the front of the eye (see section 2.3.3).

\subsubsection{Doses to ocular structures}

Currently, dosimetry for ocular brachytherapy often involves discussion of doses at points of interest (using the TG-43 formalism) rather than a volume-based approach. However, the development of a full eye model allows for investigation of dosimetry in terms of doses to volumes representing ocular structures of interest. In the current section, maximum, minimum and average doses to ocular volumes of interest, and doses to points in the eye commonly used in the literature ${ }^{44}$ are presented (Table 2.3). Dose volume histograms (DVH), which show the percent of the volume of interest (on the $y$ axis) receiving a dose greater than or equal to the corresponding dose (on the 


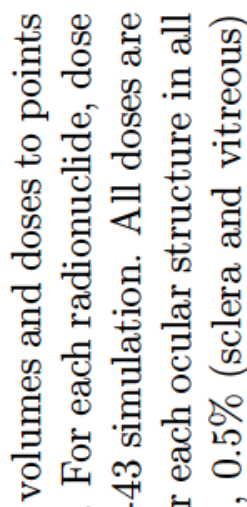

○ अ

웡

\& 0.

क्षे

设策

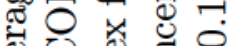

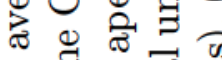

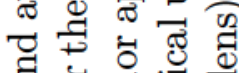

สี

घ छ

药 击

当高志志芯

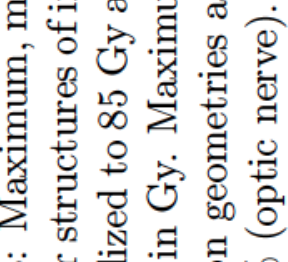

ஸ்

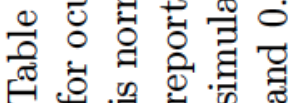

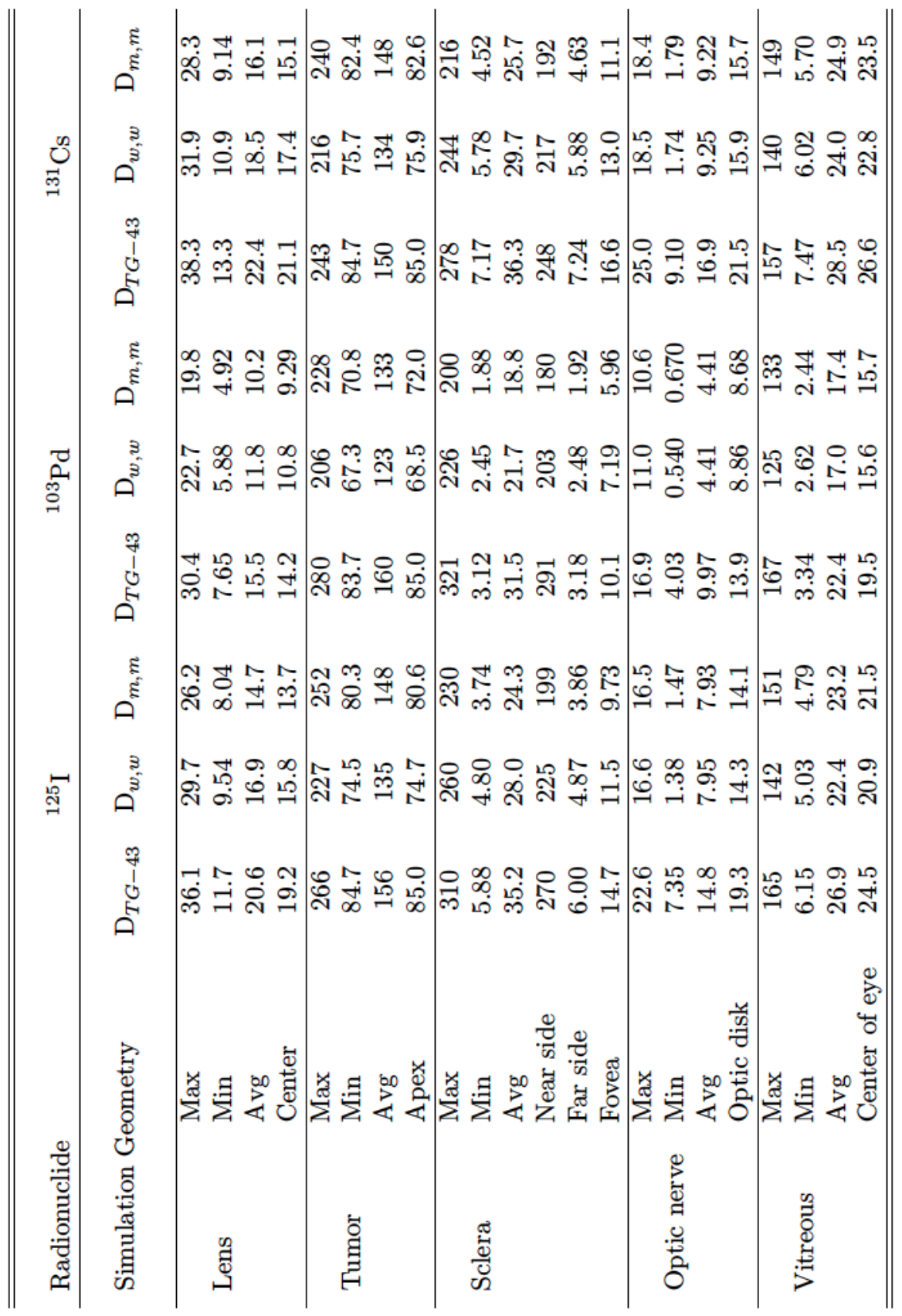




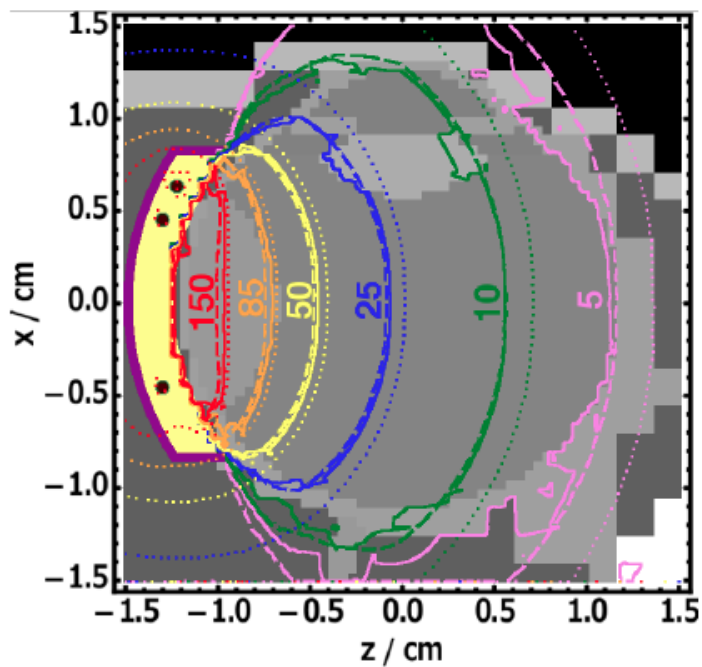

(a)

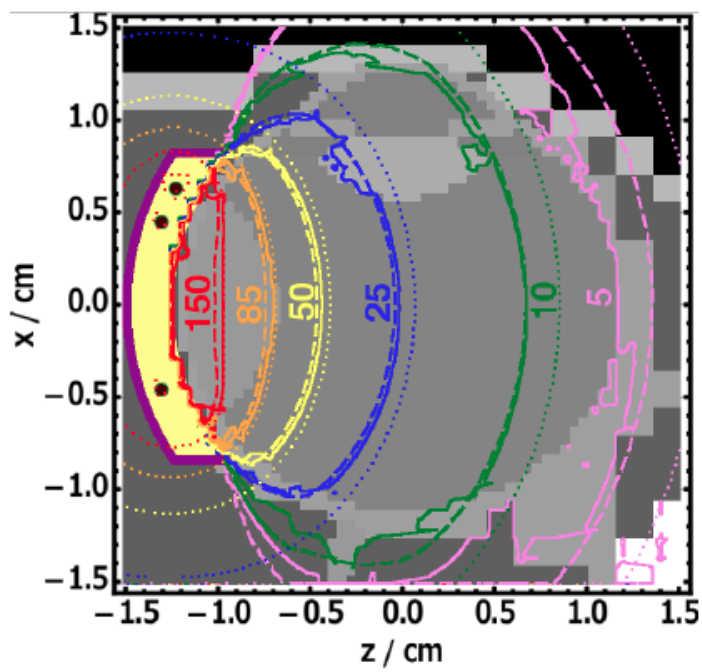

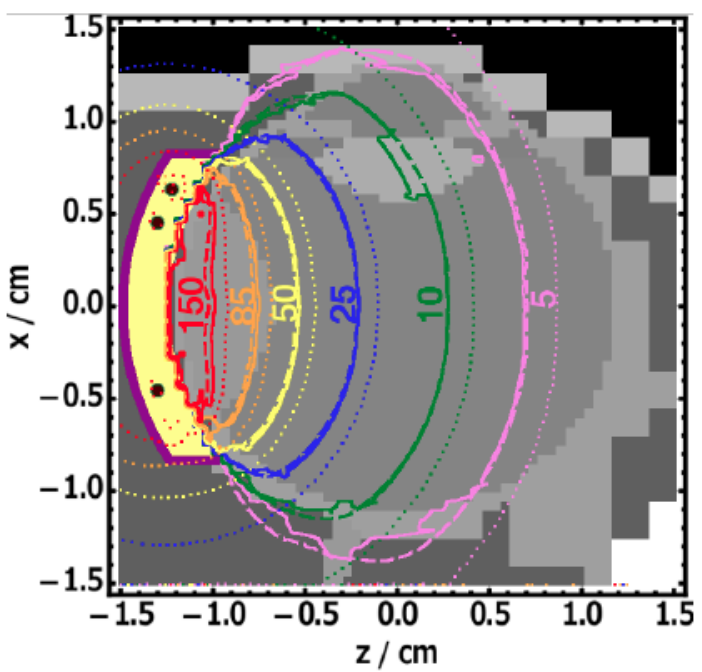

(b)

(c)

Figure 2.4: Isodose contours for (a) ${ }^{125} \mathrm{I}$, (b) ${ }^{103} \mathrm{Pd}$, and (c) ${ }^{131} \mathrm{Cs}$ from TG-43 (dotted, $\mathrm{D}_{T G-43}$ ), plaque in water (dashed, $\mathrm{D}_{w, w}$ ) and plaque in full eye model (solid, $\mathrm{D}_{m, m}$ ) simulations for the COMS plaque. Doses in Gy are indicated and are calculated using an air kerma strength to achieve a dose of 85 Gy to the tumor apex in the TG-43 simulation for the corresponding radionuclide. The central slice in the $x z$ plane is shown (voxel centers in this slice lie at $y=0.025$ $\mathrm{cm}$ as voxel boundaries lie at $y=0 \mathrm{~cm}$ ). 
$x$ axis), are also presented for the tumor and lens (Fig. 2.5 and 2.6, respectively). Isodose contours for $\mathrm{D}_{T G-43}, \mathrm{D}_{w, w}$ and $\mathrm{D}_{m, m}$ are also presented (Fig. 2.4) to provide an overview of the dose distribution within the eye.

Doses in this section are calculated assuming the same air kerma strength in all simulations for a given radionuclide; the air kerma strength used provides a dose of 85 Gy at the tumor apex for the corresponding TG-43 simulation $\left(\mathrm{D}_{T G-43}\right)$.

\section{Tumor}

Tumor doses (Fig. 2.5) vary with radionuclide and the geometry modeled. Tumor DVHs for the full eye model $\left(\mathrm{D}_{m, m}\right)$ are closer to those for the TG-43 simulations $\left(\mathrm{D}_{T G-43}\right)$ than are those for the plaque in water simulations $\left(\mathrm{D}_{w, w}\right)$; isodose contours (Fig. 2.4) also show $\mathrm{D}_{m, m}$ to be higher than $\mathrm{D}_{w, w}$ and lower than $\mathrm{D}_{T G-43}$ in the tumor region. These results demonstrate that the dose reduction due to the presence of the plaque and interseed effects is counteracted by higher mass energy absorption coefficients in the tumor than in water for the relevant photon energies.

The average $\mathrm{D}_{m, m}$ to the tumor for all radionuclides (Table 2.3) ranges from $2-17 \%$ lower and 8-10\% higher than the average $\mathrm{D}_{T G-43}$ and $\mathrm{D}_{w, w}$, respectively. The largest difference between $\mathrm{D}_{T G-43}$ and $\mathrm{D}_{m, m}$ is for ${ }^{103} \mathrm{Pd}$ due to significant reduction in dose resulting from plaque effects, consistent with other studies that show a lower $\mathrm{D}_{T G-43}$ to $\mathrm{D}_{w, w}$ ratio for ${ }^{103} \mathrm{Pd}$ than for ${ }^{125} \mathrm{I}$ in this region. ${ }^{44,48}$ Overall, the ${ }^{103} \mathrm{Pd}$ seeds produce the lowest average $\mathrm{D}_{m, m}, 133 \mathrm{~Gy}$, whereas the other two radionuclides provide an average $\mathrm{D}_{m, m}$ of $148 \mathrm{~Gy}$.

The tumor apex dose is lower for the full eye model $\left(\mathrm{D}_{m, m}\right)$ than for TG-43 $\left(\mathrm{D}_{T G-43}\right)$ by $15 \%, 5 \%$ and $3 \%$ for ${ }^{103} \mathrm{Pd},{ }^{125} \mathrm{I}$ and ${ }^{131} \mathrm{Cs}$, respectively, whereas $\mathrm{D}_{m, m}$ is larger than the dose for the plaque in water $\left(\mathrm{D}_{w, w}\right)$ by $5 \%, 8 \%$ and $9 \%$, respectively 


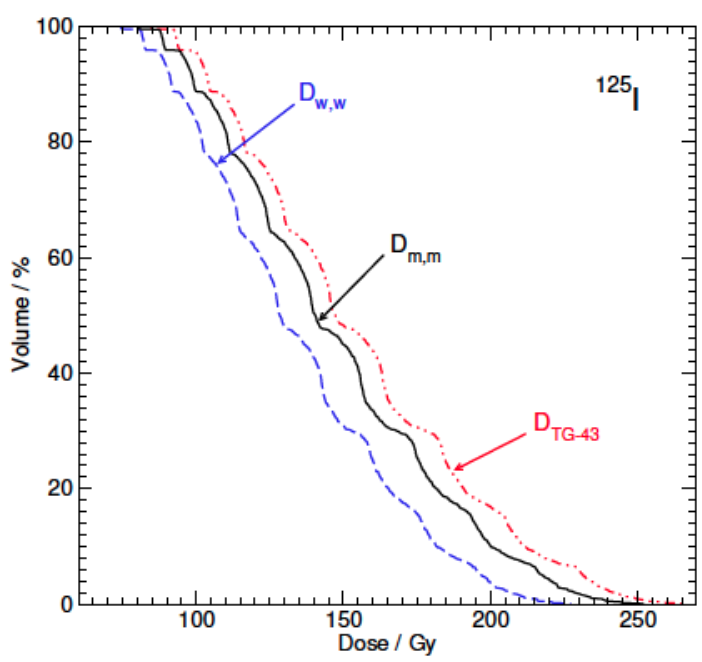

(a)

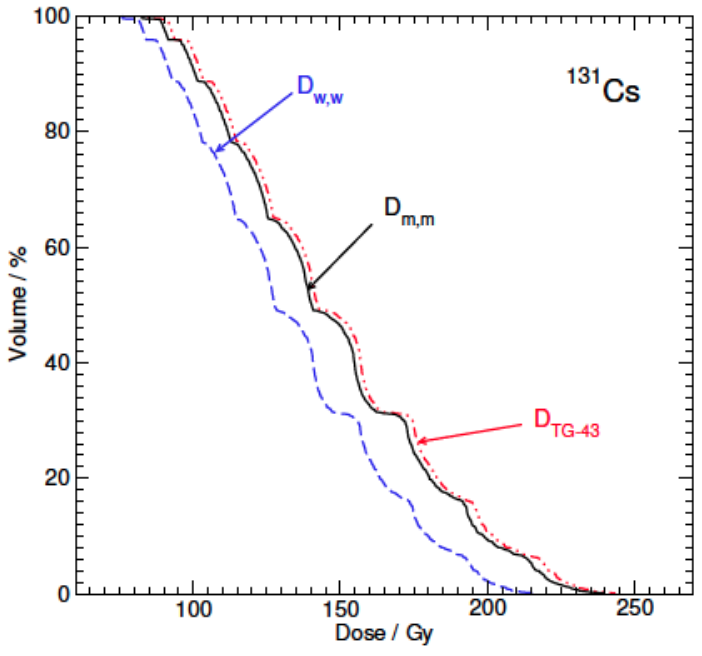

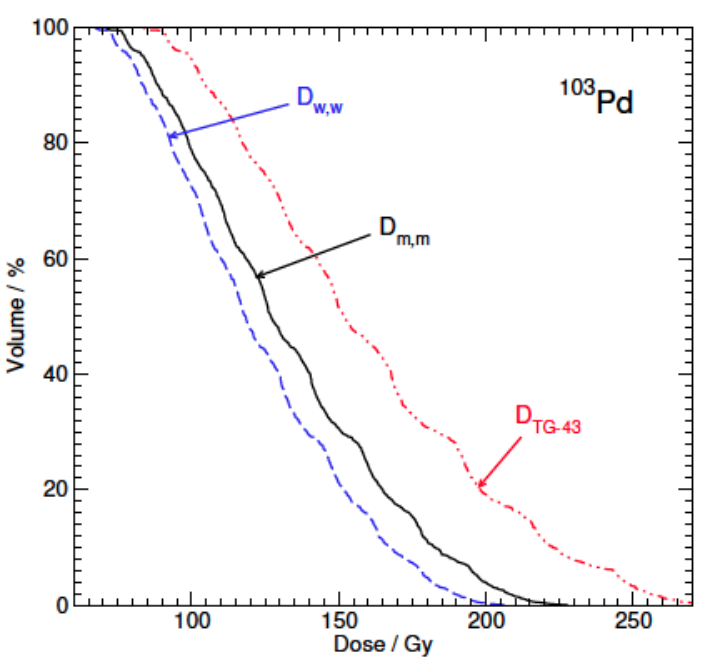

(b)

(c)

Figure 2.5: Dose volume histograms for (a) ${ }^{125} \mathrm{I}$, (b) ${ }^{103} \mathrm{Pd}$, and (c) ${ }^{131} \mathrm{Cs}$ for the tumor region from TG-43 $\left(\mathrm{D}_{T G-43}\right)$, plaque in water $\left(\mathrm{D}_{w, w}\right)$, and plaque in full eye model $\left(\mathrm{D}_{m, m}\right)$ simulations for the COMS plaque. Doses in each simulation are calculated using the air kerma strength which results in a dose of $85 \mathrm{~Gy}$ to the tumor apex in the TG-43 simulation for the corresponding radionuclide. The observed ripples of the curves are caused by the voxelization of the tumor, as voxels a given distance along the plaque's central axis have similar absorbed doses (due to dose conformity from multiple seeds). 
(Table 2.3). The minimum $\mathrm{D}_{m, m}$ in the tumor volume is within $1 \%$ for ${ }^{125} \mathrm{I}$ and ${ }^{131} \mathrm{Cs}$ and $2 \%$ for ${ }^{103} \mathrm{Pd}$ of the dose to its apex in all simulation geometries (as expected since the apex is the furthest point from the plaque).

\section{Lens}

For the lens, $\mathrm{D}_{m, m}$ is generally lower than $\mathrm{D}_{T G-43}$ and $\mathrm{D}_{w, w}$ (Figs. 2.4, 2.6). From Table 2.3, average $\mathrm{D}_{m, m}$ to the lens is lower than the average $\mathrm{D}_{T G-43}$ to the lens by $29 \%, 34 \%$ and $28 \%$ for ${ }^{125} \mathrm{I},{ }^{103} \mathrm{Pd}$ and ${ }^{131} \mathrm{Cs}$, respectively, and is lower than the average lens $\mathrm{D}_{w, w}$ by $13-14 \%$ for all three radionuclides. The lower dose in the lens medium compared with the two water simulations occurs as a result of the reduced photon fluence in the lens due to radiation transport through the tumor and vitreous, the lack of backscatter due to the presence of an air interface in the eye model, and lower mass energy absorption coefficients for lens than water. In general, doses are lowest for ${ }^{103} \mathrm{Pd}$ and highest for ${ }^{131} \mathrm{Cs}$.

Dose to the center of the lens is lower than the average dose to the lens in all simulation geometries $\left(\mathrm{D}_{m, m}, \mathrm{D}_{T G-43}\right.$ and $\left.\mathrm{D}_{w, w}\right)$ by $9 \%, 7 \%$ and $6 \%$ for ${ }^{103} \mathrm{Pd},{ }^{125} \mathrm{I}$ and ${ }^{131} \mathrm{Cs}$, respectively (Table 2.3). In all simulation geometries, the maximum dose to the lens is roughly twice the dose at center of lens for all radionuclides. As the lens is a radiosensitive structure, the maximum dose may be important and its value is not evident from dose reporting to the central voxel. Furthermore, research suggests that the most radiosensitive cells lie in the germinative epithelium of the lens (in the lens' equatorial region near its anterior face $)^{83}$ rather than at the center of the lens. 

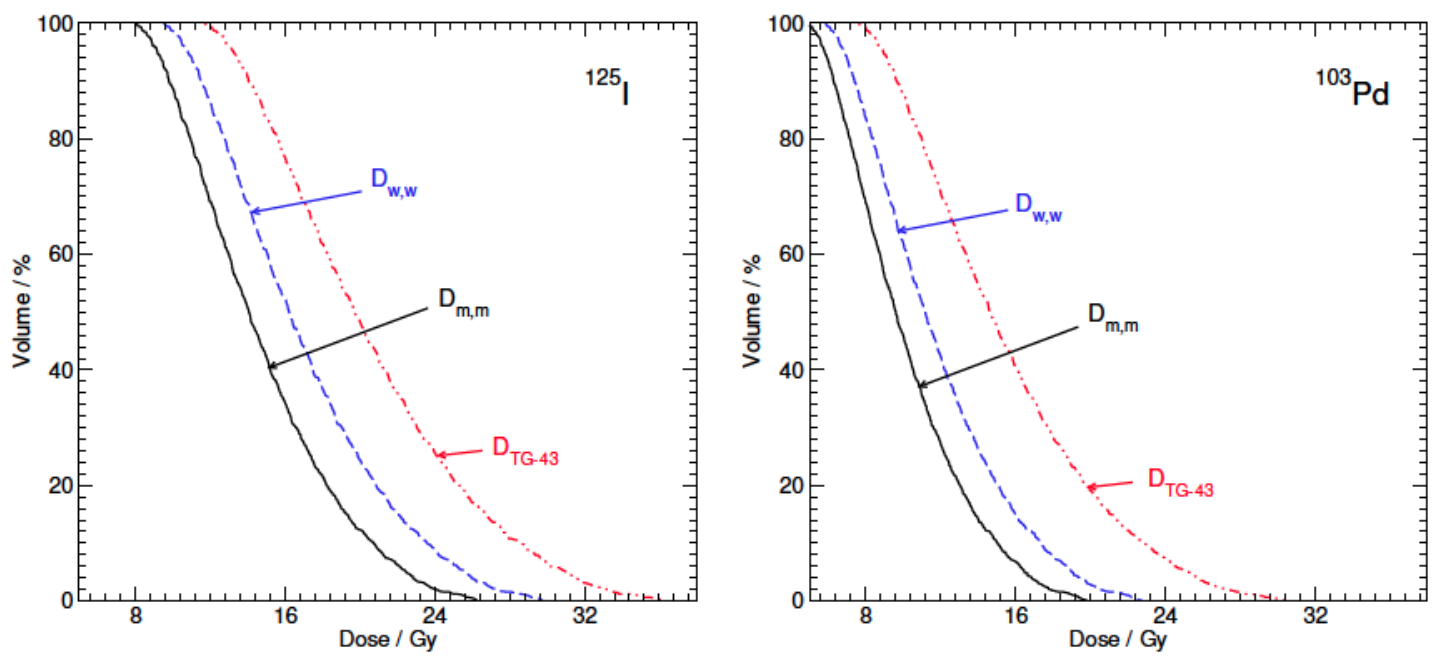

(a)

(b)

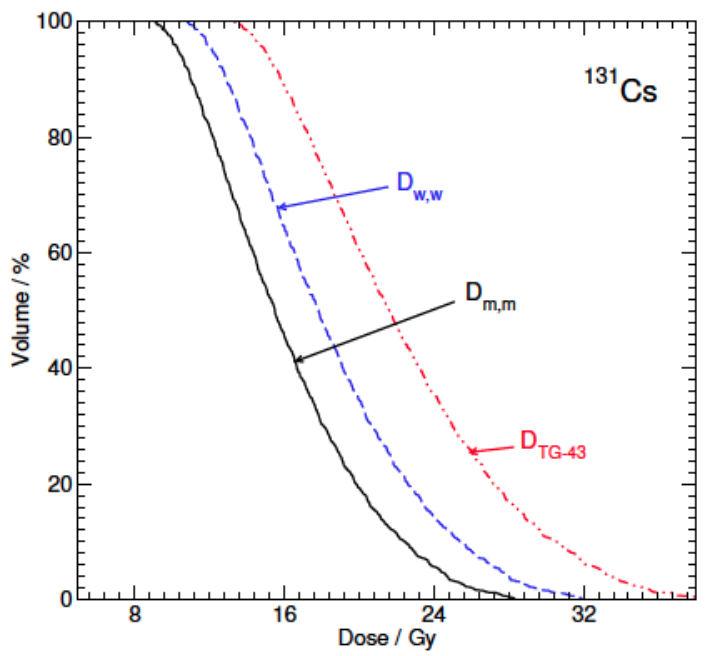

(c)

Figure 2.6: Dose volume histogram for (a) ${ }^{125} \mathrm{I}$, (b) ${ }^{103} \mathrm{Pd}$, and (c) ${ }^{131} \mathrm{Cs}$ for the lens region from TG-43 $\left(\mathrm{D}_{T G-43}\right)$, plaque in water $\left(\mathrm{D}_{w, w}\right)$ and plaque in full eye model $\left(\mathrm{D}_{m, m}\right)$ simulations for the COMS plaque. Doses in each simulation are calculated using the air kerma strength which results in a dose of 85 Gy to the tumor apex in the TG-43 simulation for the corresponding radionuclide. 


\section{Vitreous}

For the vitreous region, the average $\mathrm{D}_{m, m}$ is closer to that for $\mathrm{D}_{w, w}$ than for $\mathrm{D}_{T G-43}$ : average $\mathrm{D}_{m, m}$ is $22 \%, 14 \%$ and $12 \%$ less than the average $\mathrm{D}_{T G-43}$, and $2 \%, 4 \%$ and $4 \%$ more than the average $\mathrm{D}_{w, w}$ for ${ }^{103} \mathrm{Pd},{ }^{125} \mathrm{I}$ and ${ }^{131} \mathrm{Cs}$, respectively (Table 2.3 ). The dose to the center of the eye is not representative of the average dose to the vitreous; the dose at the eye's center is lower than the average dose to the vitreous by $5-7 \%$ for the ${ }^{131}$ Cs simulations, $7-9 \%$ for the ${ }^{125}$ I simulations and $8-13 \%$ for the ${ }^{103} \mathrm{Pd}$ simulations (where the highest values within the presented ranges correspond with the TG-43 simulations and the lowest values correspond to simulations with the plaque in water). Saccadic rotations of the eye cause vitreous mixing; due to the complexity of vitreous flow ${ }^{84}$ and the shorter timescales of mixing compared to treatment times, maximum and minimum calculated doses to this structure may not be representative of the true values. Thus, the average dose to the vitreous may be a more meaningful quantity than the central eye dose.

\section{Sclera}

As the near side of the sclera is directly adjacent to the plaque and has a single voxel thickness, the fluence in the voxels adjacent to the plaque is similar for the plaque in water and the full eye model simulations. Hence, the difference between $\mathrm{D}_{m, m}$ and $\mathrm{D}_{w, w}$ (Table 2.3) is roughly given by the ratio of mass energy absorption coefficients of the sclera medium to water (averaged over the appropriate photon energy spectrum). Average scleral $\mathrm{D}_{m, m}$ and $\mathrm{D}_{w, w}$ differ slightly more because voxels further from the plaque experience differences in fluence due to radiation transport through ocular media as opposed to water.

The sclera has a large range of doses and the average dose is significantly different 
than the maximum dose (Table 2.3). Comparison of the maximum scleral dose to the relevant point of interest at the near side of the sclera (as shown in Fig. 2.1(a)) in all simulation geometries $\left(\mathrm{D}_{T G-43}, \mathrm{D}_{w, w}\right.$ and $\left.\mathrm{D}_{m, m}\right)$ indicates a $12 \%$ difference for ${ }^{131} \mathrm{Cs}$, $15 \%$ for ${ }^{125} \mathrm{I}$, and $10-11 \%$ for ${ }^{103} \mathrm{Pd}$; the minimum scleral dose and the dose at the furthest scleral voxel from the plaque are within $3 \%$ of each other for all simulations and all radionuclides.

\section{Optic Nerve}

For the optic nerve, the maximum $\mathrm{D}_{m, m}$ is smaller than the maximum $\mathrm{D}_{T G-43}$ by $37 \%$ for ${ }^{103} \mathrm{Pd}, 27 \%$ for ${ }^{125} \mathrm{I}$ and $26 \%$ for ${ }^{131} \mathrm{Cs}$. For the optic nerve, the maximum $\mathrm{D}_{w, w}$ and $\mathrm{D}_{m, m}$ are within statistical uncertainty of each other for ${ }^{125} \mathrm{I}$ and ${ }^{131} \mathrm{Cs}$; the maximum $\mathrm{D}_{m, m}$ is smaller than the maximum $\mathrm{D}_{T G-43}$ by $4 \%$ for ${ }^{103} \mathrm{Pd}$ (Table 2.3). ${ }^{103} \mathrm{Pd}$ produces a larger difference between maximum $\mathrm{D}_{w, w}$ and $\mathrm{D}_{m, m}$ due to additional attenuation of ${ }^{103} \mathrm{Pd}$ photons in the eye model compared with the other radionuclides; the mass energy absorption coefficient ratio of optic nerve to water media is also slightly lower near the average photon energy of ${ }^{103} \mathrm{Pd}(\sim 21 \mathrm{keV})$ than near the average photon energies of ${ }^{125} \mathrm{I}$ and ${ }^{131} \mathrm{Cs}(\sim 27$ and $30 \mathrm{keV}$, respectively).

Maximum $\mathrm{D}_{m, m}$ for the optic nerve is larger than $\mathrm{D}_{m, m}$ to the center of the optic disk for all radionuclides by $17-22 \%$, with the largest difference occurring for

${ }^{103} \mathrm{Pd}$. The maximum dose occurs in an optic nerve voxel lying closer to the plaque than the optic disk. As with the lens, the optic nerve is considered radiosensitive; thus, accurate knowledge of maximum dose may be important and is not properly represented by the dose to the center of the optic disk. 


\subsubsection{Sensitivity of doses to tumor composition, surrounding tissues, and plaque position}

Tumor composition affects the fluence exiting the tumor and consequently doses to ocular structures. Fig. 2.7 presents doses for the ${ }^{125} \mathrm{I}$ simulations with the alternate tumor materials relative to the default full simulation along the tumor $(z)$ axis: doses within the tumor are up to $5 \%$ higher with "melanoma 2 " and lower by up to $8 \%$ with water and $16 \%$ with soft tissue. Differences in vitreous doses (Fig. 2.7) due to changes in the photon fluence induced by the tumor composition are within $1-2.5 \%$.

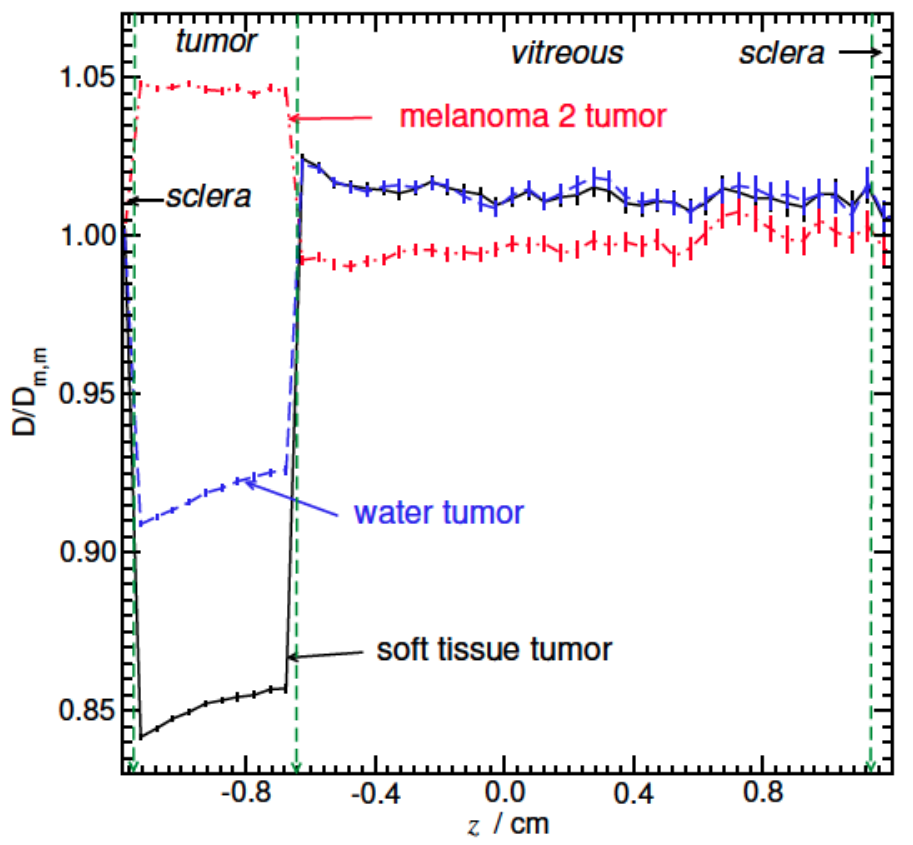

Figure 2.7: ${ }^{125} \mathrm{I}$ dose ratio along the $z$ axis (voxel centers are at $x=0.025 \mathrm{~cm}$ and $y=0.025 \mathrm{~cm}$ as the voxel boundaries are at $x=0 \mathrm{~cm}$ and $y=0.025 \mathrm{~cm}$ ) from simulations of the COMS plaque in the full eye model but with the tumor material replaced with: group III male soft tissue from White et al., ${ }^{82}$ water, and the second melanoma composition listed in Maughan et al. ${ }^{73}$ to the full eye model used in this study $\left(\mathrm{D}_{m, m}\right)$. Doses are calculated using the same air kerma strength for all simulations.

For all tumor media substitutions, maximum, minimum and average doses to all 
structures studied in this work (not shown), with the exception of the tumor itself, are within $1 \%$ of the values presented in section 2.3.2. Maximum, minimum and average doses to the tumor vary by $4-16 \%$ with this replacement, with the largest difference found in replacing the tumor with soft tissue and the smallest with melanoma 2 . Thus, dose throughout the eye is not largely affected by tumor composition; however, doses to the tumor are significantly affected by its composition so care must be taken if dose is normalized based on the dose to the tumor.

Media surrounding the eye affect dose within the eye due to backscatter, thus dose sensitivity to surrounding media is investigated for ${ }^{125} \mathrm{I}$. Dose ratios in the $z$ direction at $x=0.825 \mathrm{~cm}$ (near the front of the eye) for the eye model in water with and without a flat anterior air interface to the eye model in the ICRP computational phantom $\left(\mathrm{D}_{m, m}\right)$ are shown in Fig. 2.8(a). For the eye model in water with and without the air interface, doses along this line within the eye model are up to $3 \%$ and up to $9 \%$ larger than $\mathrm{D}_{m, m}$, respectively; the largest increase is for the furthest voxels from the plaque along this line as a result of decreased backscatter in this region for $\mathrm{D}_{m, m}$ (due to air which wraps around the side of the head in the computational phantom which is not present with the flat air interface in the water phantom). Fig. 2.8(b) shows the same dose ratio in the $z$ direction as for Fig. 2.8(a) but at $x=-1.075 \mathrm{~cm}$, in a region near bone. Within the eye, doses for the eye in water with and without an air interface along this line are within $2 \%$ of $\mathrm{D}_{m, m}$; doses within the eye model are therefore not significantly affected by the presence of bone.

The replacement of the tissues surrounding the eye model with water (with and without an air interface) for ${ }^{125}$ I generally results in negligible differences in maximum, minimum and average doses $(<1 \%)$ for most ocular structures in comparison with the full eye model with the computational phantom as surrounding tissue, with a few exceptions. With only water (no air interface) surrounding the eye model, dose 

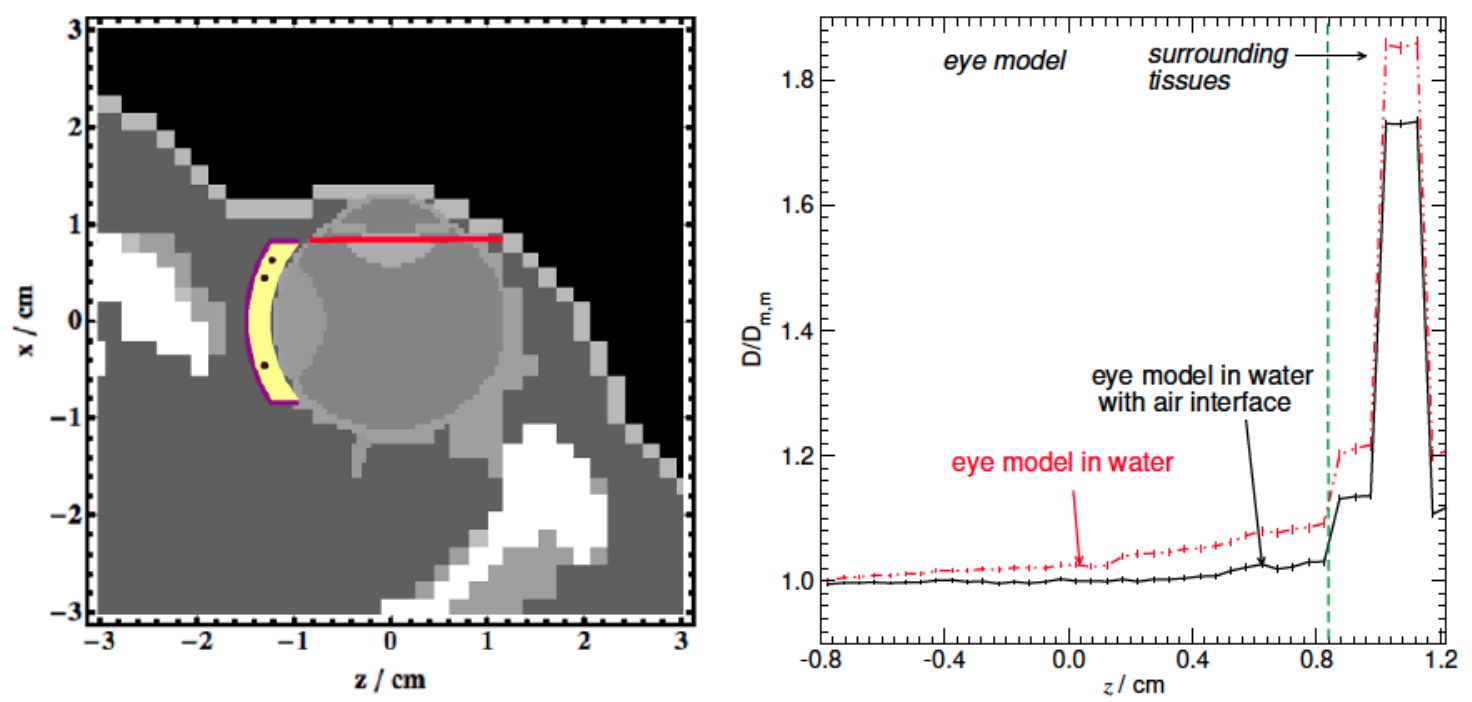

(a)

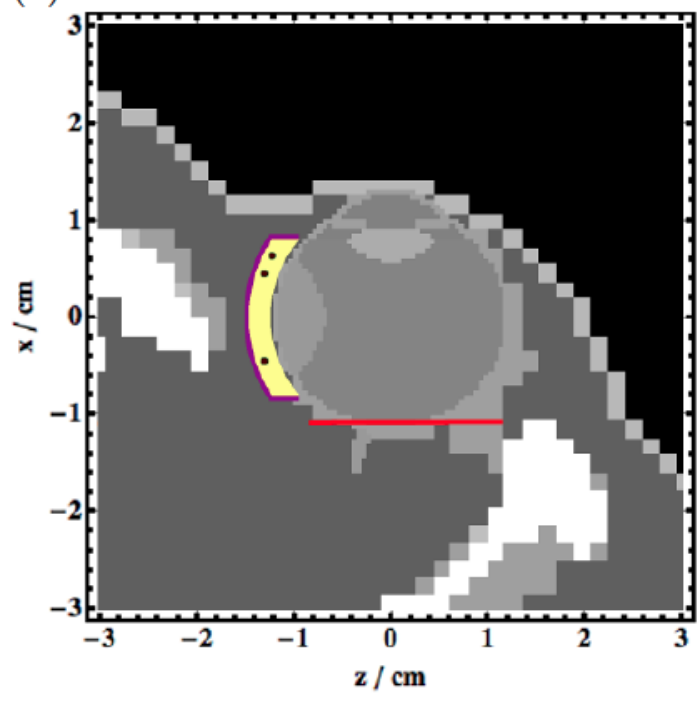

(b)

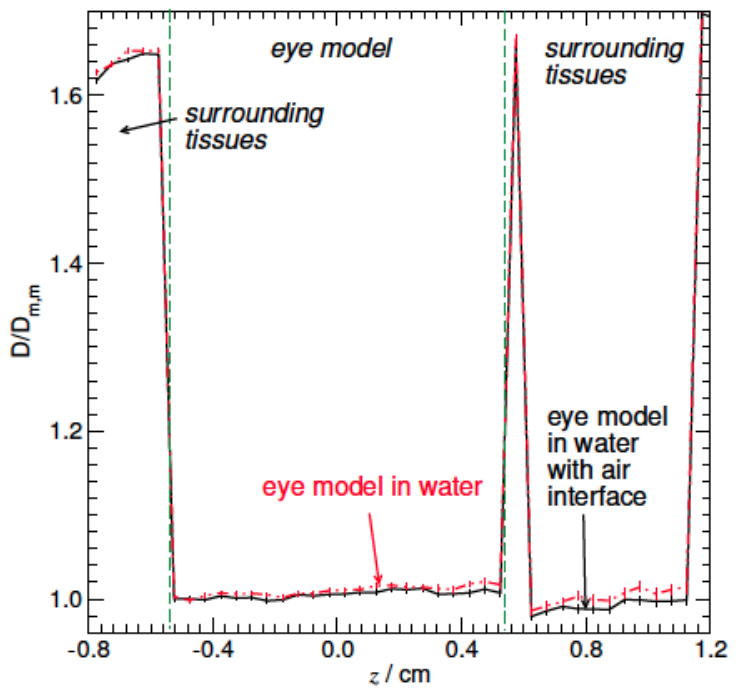

(d)

(c)

Figure 2.8: Dose ratios in the $z$ direction for ${ }^{125} \mathrm{I}$ at (b) $x=0.825 \mathrm{~cm}$ and (d) $x=-1.075 \mathrm{~cm}$ (profiles depicted in (a) and (c), respectively) from simulations of the COMS plaque in the eye model surrounded by water with or without an air interface to the same plaque in the full eye model $\left(\mathrm{D}_{m, m}\right)$ with heterogeneous surrounding tissues of: (b) homogeneous eye material ( $z=0.85$ to $1 \mathrm{~cm}$ ), adipose $(z=1$ to $1.1 \mathrm{~cm})$ and skin $(z=1.1$ to $1.2 \mathrm{~cm})$, and (d) adipose $(z=-0.8$ to $-0.55 \mathrm{~cm}$, $z=0.55$ to $0.6 \mathrm{~cm}$ and $z=1.15$ to $1.2 \mathrm{~cm}$ ), and muscle $(z=0.6$ to $1.15 \mathrm{~cm})$. Doses are calculated using the same air kerma strength for all simulations. 
at the far side of the sclera is $6 \%$ larger, lens maximum and average doses are $2-3 \%$ larger, and minimum dose to the vitreous is $8 \%$ larger than for the full eye model. With water and a planar air interface, these doses are in closer agreement with those for the full eye model with differences of $3 \%$ at the far side of the sclera and $5 \%$ in the minimum vitreous dose; lens doses are in agreement. These results suggest that detailed modeling of tissues surrounding the eye may not be necessary for clinical applications where only doses to ocular structures are of interest. Fig. 2.8 shows significant differences between doses to tissues surrounding the full eye model and doses to water surrounding the eye, due to differences in fluence and in the mass energy absorption coefficients of the surrounding media and water.

This chapter focuses on a single tumor/plaque position on the nasal side of the eye; however, two other positions are also modeled (section 2.2.1): a tumor centered along the $z$ axis but on the lateral side rather than the medial (nasal) side, and a tumor at the eye's posterior centered along the $x$ axis. For the posterior tumor, the optic nerve is not simulated as the plaque would overlap it in this position. $\mathrm{D}_{m, m}$ to ocular structures in the posterior tumor position is affected by the $1 \mathrm{~mm}$ (2 voxels) scleral thickness in this region: the tumor apex lies further from the plaque (tumor apex lies $5 \mathrm{~mm}$ inwards from the inner sclera), thus normalization to 85 Gy at the tumor apex for TG-43 increases dose throughout the eye compared with the medial location. As a result, $\mathrm{D}_{m, m}$ at the posterior tumor's apex differs by $10 \%$ in comparison to the $\mathrm{D}_{m, m}$ to the tumor apex for the nasal tumor position; also, the average scleral $\mathrm{D}_{m, m}$ is nearly doubled for the posterior as opposed to the nasal tumor position (the larger number of scleral voxels near the plaque in the posterior tumor position contributes to this increase). The increased distance between the plaque and the lens with the posterior tumor results in a $46 \%$ lower average lens $\mathrm{D}_{m, m}$ than with the nasal tumor.

$\mathrm{D}_{m, m}$ to most structures is less affected by the repositioning of the tumor and 
plaque to the distal side of the eye, opposite the nasal location, as all structures apart from the optic nerve are symmetric about the $x$ axis. The largest differences in dose are observed in the optic nerve, as it lies further from the plaque in the distal tumor position; the maximum optic nerve dose is $43 \%$ lower for the distal compared to the nasal position.

\subsubsection{Comparison of radionuclides}

Doses delivered using each radionuclide are compared by representing doses as percentages of the dose to the tumor apex, henceforth denoted as $\% \mathrm{D}_{\text {apex }}$ (Fig. 2.9, Table 2.4). Focusing on results for the full eye model $\left(\mathrm{D}_{m, m}\right)$, average tumor values of $\% \mathrm{D}_{\text {apex }}$ (Table 2.4 ) differ by less than $5 \% \mathrm{D}_{\text {apex }}$ between all radionuclides, with the lowest value for ${ }^{131} \mathrm{Cs}$ and highest for ${ }^{103} \mathrm{Pd}$. The average and maximum lens $\% \mathrm{D}_{\text {apex }}$ values for ${ }^{131} \mathrm{Cs}$ are larger by $1-2 \% \mathrm{D}_{\text {apex }}$ than those for ${ }^{125} \mathrm{I}$, while these doses are smaller by $4-6 \% \mathrm{D}_{\text {apex }}$ for ${ }^{103} \mathrm{Pd}$ than for ${ }^{125} \mathrm{I}$. Vitreous maximum $\% \mathrm{D}_{\text {apex }}$ is lower by 7 $\% \mathrm{D}_{\text {apex }}$ for ${ }^{131} \mathrm{Cs}$ and $3 \% \mathrm{D}_{\text {apex }}$ for ${ }^{103} \mathrm{Pd}$ compared with ${ }^{125} \mathrm{I}$; average vitreous $\% \mathrm{D}_{\text {apex }}$ values for ${ }^{131} \mathrm{Cs}$ and ${ }^{125} \mathrm{I}$ are within roughly $1 \% \mathrm{D}_{\text {apex }}$ of each other, while the value for ${ }^{103} \mathrm{Pd}$ is lower by $5 \% \mathrm{D}_{\text {apex }}$ than for ${ }^{125} \mathrm{I}$. Maximum scleral $\% \mathrm{D}_{\text {apex }}$ is highest for ${ }^{131} \mathrm{Cs}$ and lowest for ${ }^{103} \mathrm{Pd}$. Optic nerve maximum and average values of $\% \mathrm{D}_{\text {apex }}$ are lower by $5 \% \mathrm{D}_{\text {apex }}$ and $4 \% \mathrm{D}_{\text {apex }}$, respectively, for ${ }^{103} \mathrm{Pd}$ than for ${ }^{125} \mathrm{I}$; these values are within $2 \% \mathrm{D}_{\text {apex }}$ for ${ }^{131} \mathrm{Cs}$ and ${ }^{125} \mathrm{I}$. The observed differences in dose distributions (in the full eye model) between radionuclides may be particularly important in the radiosensitive lens and optic nerve. 


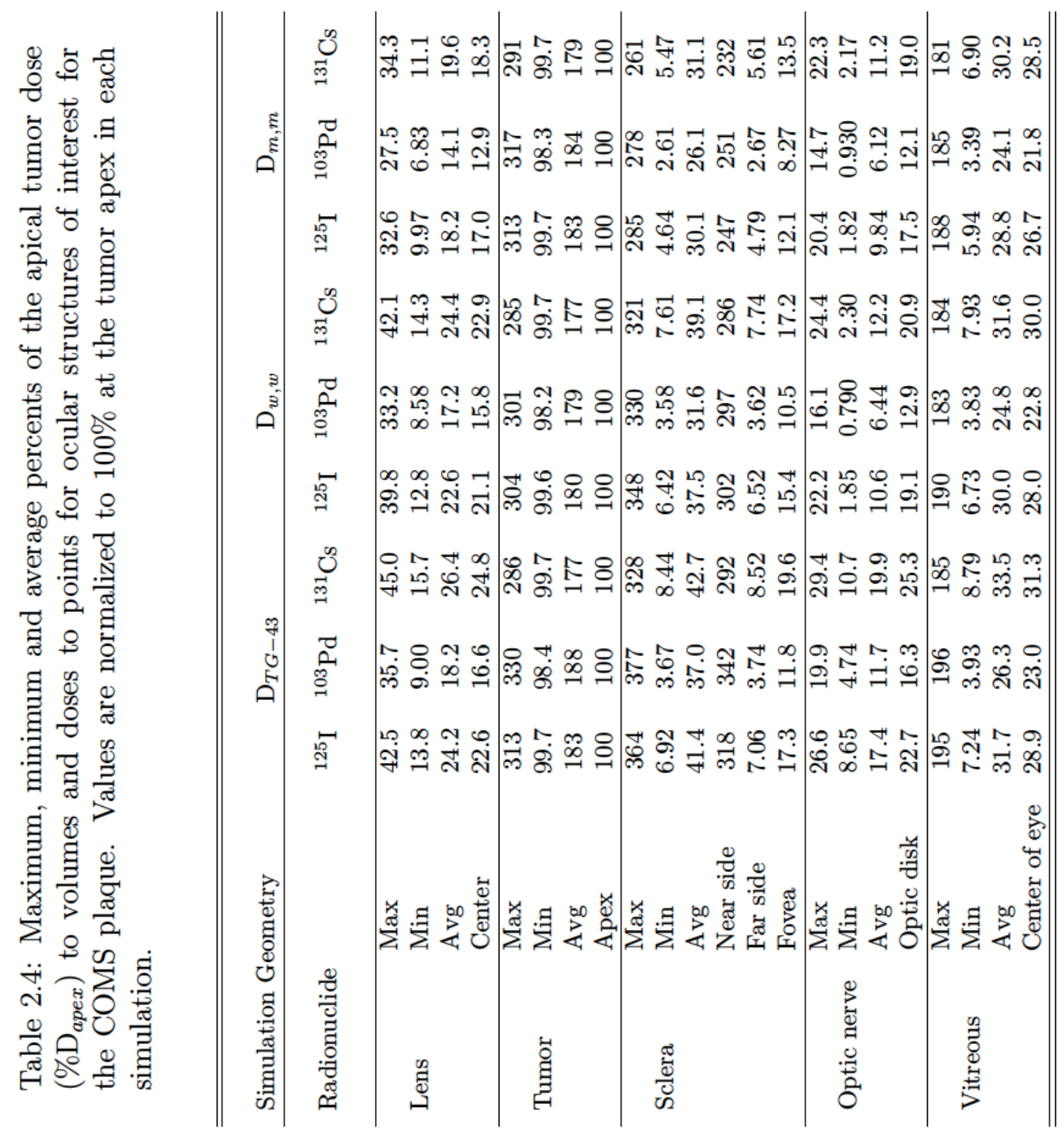




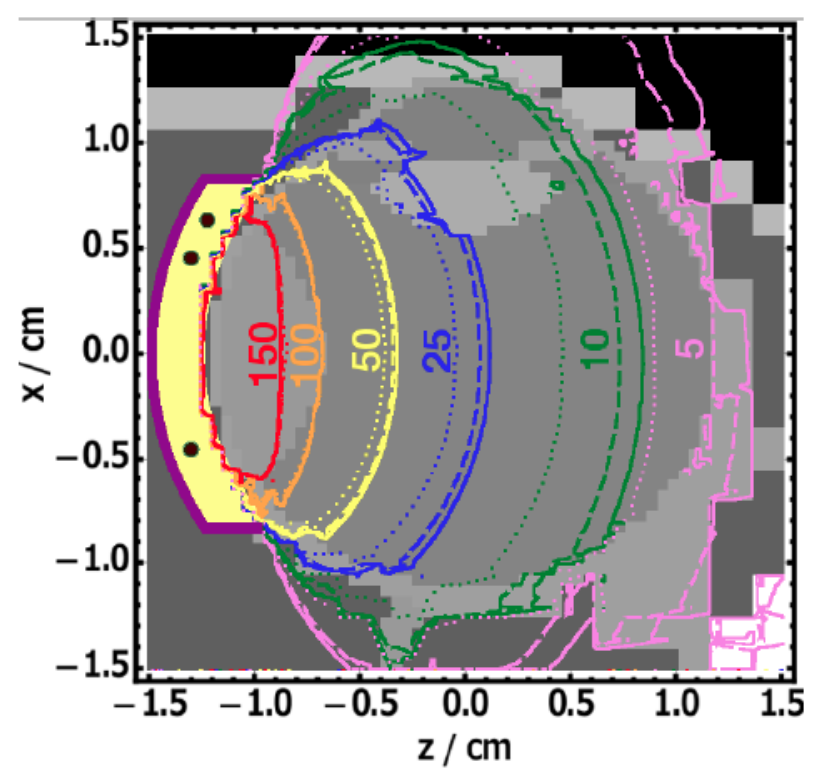

Figure 2.9: Percentage isodose contours $\left(\% \mathrm{D}_{\text {apex }}\right)$ for $\mathrm{D}_{m, m}$ for ${ }^{125} \mathrm{I}$ (dashed), ${ }^{103} \mathrm{Pd}$ (dotted) and ${ }^{131} \mathrm{Cs}$ (solid) within the COMS plaque. For each radionuclide, doses are normalized to $100 \%$ at the tumor apex, with values indicated. The central slice in the $x z$ plane is shown (voxel centers in this slice lie at $y=0.025$ $\mathrm{cm}$ as voxel boundaries lie at $y=0 \mathrm{~cm}$ ).

The comparison of dose distributions for the different radionuclides depends on the choice of simulation geometry due to differences in plaque and ocular media effects between radionuclides. Most noticeably, using the TG-43 geometry, the maximum tumor $\% \mathrm{D}_{\text {apex }}$ for ${ }^{103} \mathrm{Pd}$ is higher by $17 \% \mathrm{D}_{\text {apex }}$ than for ${ }^{125} \mathrm{I}$, whereas this value is lower by $3 \% \mathrm{D}_{\text {apex }}$ (higher by $4 \% \mathrm{D}_{\text {apex }}$ ) for ${ }^{103} \mathrm{Pd}$ than ${ }^{125} \mathrm{I}$ using the plaque in water (full eye model). Similarly, the average tumor $\% \mathrm{D}_{\text {apex }}$ is higher by $5 \% \mathrm{D}_{\text {apex }}$ with ${ }^{103} \mathrm{Pd}$ than with ${ }^{125} \mathrm{I}$ in the TG-43 simulations but the average tumor $\% \mathrm{D}_{\text {apex }}$ is within $1 \% \mathrm{D}_{\text {apex }}$ for these radionuclides using the other two simulation geometries. Comparison of scleral and vitreous doses for different radionuclides is also sensitive to the simulation geometry. 


\subsection{Conclusion}

In this chapter, a rectilinear voxelized representative eye model with realistic geometry and composition was developed. Using a $16 \mathrm{~mm}$ COMS plaque, it was found that ocular media play a significant role in dose calculations for eye plaque brachytherapy, and that dose differences between the plaque in water $\left(\mathrm{D}_{w, w}\right)$ and the plaque in the full eye model $\left(\mathrm{D}_{m, m}\right)$ vary within the eye. Within the tumor, doses for the plaque in water $\left(\mathrm{D}_{w, w}\right)$ underestimate doses for the plaque in the full eye model $\left(\mathrm{D}_{m, m}\right)$; however, in the lens, $\mathrm{D}_{w, w}$ overestimates the dose by as much as $13-14 \%$ for all radionuclides. For the optic nerve, the maximum $\mathrm{D}_{w, w}$ is within the statistical uncertainty of the maximum $\mathrm{D}_{m, m}$ for ${ }^{125} \mathrm{I}$ and ${ }^{131} \mathrm{Cs}$; although the maximum $\mathrm{D}_{w, w}$ and $\mathrm{D}_{m, m}$ differ for ${ }^{103} \mathrm{Pd}$ by $4 \%$. Differences between average doses to the tumor and lens regions in the full eye model $\left(\mathrm{D}_{m, m}\right)$ and the TG-43 simulation $\left(\mathrm{D}_{T G-43}\right)$ are also significant, as they differ by $2-17 \%$ for the tumor and $29-34 \%$ for the lens. Discrepancies between doses in the different simulation geometries result from the combined effects of the ocular media, interseed attenuation and the plaque.

Comparisons between doses in single voxels of interest and maximum, minimum and average doses for the COMS plaque suggest that doses to single voxels are not necessarily representative of doses within the ocular structure. In the lens, the average dose is $7-9 \%$ higher than the dose to the central voxel, while in the optic nerve the maximum dose is $17-22 \%$ higher than the dose to the optic disk for all radionuclides (for simulations of the plaque in full eye model). Maximum doses to these structures may be of particular interest as they are considered radiosensitive and are common sites for secondary effects such as optical neuropathy and cataracts ${ }^{47}$ (whereas the sclera and cornea are generally considered radioresistant $\left.{ }^{85}\right)$.

Dose distributions in this chapter are dependent on assigned media compositions, 
many of which are not accurately known for the ocular structures modeled. Tumor composition affects the dose to the tumor significantly, and can affect the dose to other structures within the eye as a result of normalization to achieve the prescribed apical tumor dose. Accurate modeling of the tissues surrounding the eye is less important than for the tissues within the eye as substituting surrounding tissues with water causes small differences in doses to most structures of interest within the eye.

Comparison of doses delivered with the different radionuclides in the full eye model, normalized in terms of dose to the tumor apex, suggest that doses delivered to critical ocular structures using the COMS plaque are generally lowest for ${ }^{103} \mathrm{Pd}$ and highest for ${ }^{131} \mathrm{Cs}$, with the exception of the tumor where average doses are comparable for ${ }^{125} \mathrm{I}$ and ${ }^{103} \mathrm{Pd}$, and are lowest for ${ }^{131} \mathrm{Cs}$. Comparisons between radionuclides are sensitive to the simulation geometry. 


\section{Chapter 3}

\section{Plaque effects on dose for representative plaque models in water and ocular media}

\subsection{Introduction}

Despite work aimed at improving dosimetry for ocular brachytherapy for a few plaque models (section 1.2), studies have mostly been conducted for the plaque within a homogeneous water phantom, neglecting the presence of ocular media; Chapter 2 reported considerable differences between doses calculated in ocular media and in water for COMS eye plaque brachytherapy. Further, many studies have only considered the effects of a single seed at the center of the plaque, ${ }^{43,86}$ despite differences in dosimetric plaque effects when multiseed arrangements (i.e., fully-loaded plaques) are modeled rather than a single seed. ${ }^{44,46}$ Finally, the plaque sizes modeled by different groups vary, making comparisons of dosimetric effects of diverse plaque models difficult.

This chapter investigates eye plaque effects on dose for a range of currently-used photon-emitting plaques by developing and calculating dose for representative plaque models. To provide consistent comparisons between the effects on dose of the plaque backing and insert geometries and compositions, each plaque (apart from one model) is $16 \mathrm{~mm}$ in diameter with the same number of seeds and same seed configuration. Doses for each plaque model are calculated using Monte Carlo simulations for the same three low-energy photon-emitting radionuclides as in Chapter 2: ${ }^{125} \mathrm{I},{ }^{103} \mathrm{Pd}$ and ${ }^{131} \mathrm{Cs}$. Doses in water for a single seed at the center of each plaque and for the multiseed arrangement within each plaque are compared with doses for the single 
seed and multiple seeds within a water phantom (TG-43) to investigate plaque effects on dose. Using the full eye model developed in Chapter 2, doses to ocular structures of interest are calculated for the fully-loaded eye plaques in the full eye model and compared to those for the same plaques in water. Thus, this chapter considers the effects of both the plaque and ocular media on dose.

\subsection{Methods}

The geometry and compositions of the representative plaque models developed for this chapter are described in section 3.2.1. Section 3.2.2 outlines the Monte Carlo simulations performed. The coordinate system, dose notation, simulation parameters, and sources studied in this chapter are the same as those for Chapter 2.

\subsubsection{Development of plaque models}

Five representative plaque models are developed and are considered in the current chapter in addition to the COMS plaque (modeled previously in Thomson et al. ${ }^{44}$ and in Chapter 2). Table 3.1 describes the geometry and composition of the backing and insert for each plaque model; collimating lip length, backing and total plaque thicknesses, and distance from the center of the eye to the seed centers are all given. Table 3.1 also lists whether each plaque model has a full insert (conforms with the outer sclera) or a thin layer of fixative to hold the seeds in place. Although these plaque models are not exact replications of those used at various institutions, they serve to approximate the effects on dose of similar plaques. The plaque parameters, coordinate system, and the bounding surfaces for a right eye model (developed in Chapter 2) are depicted in Fig. 3.1 and the plaque models are shown in Fig. 3.2. 


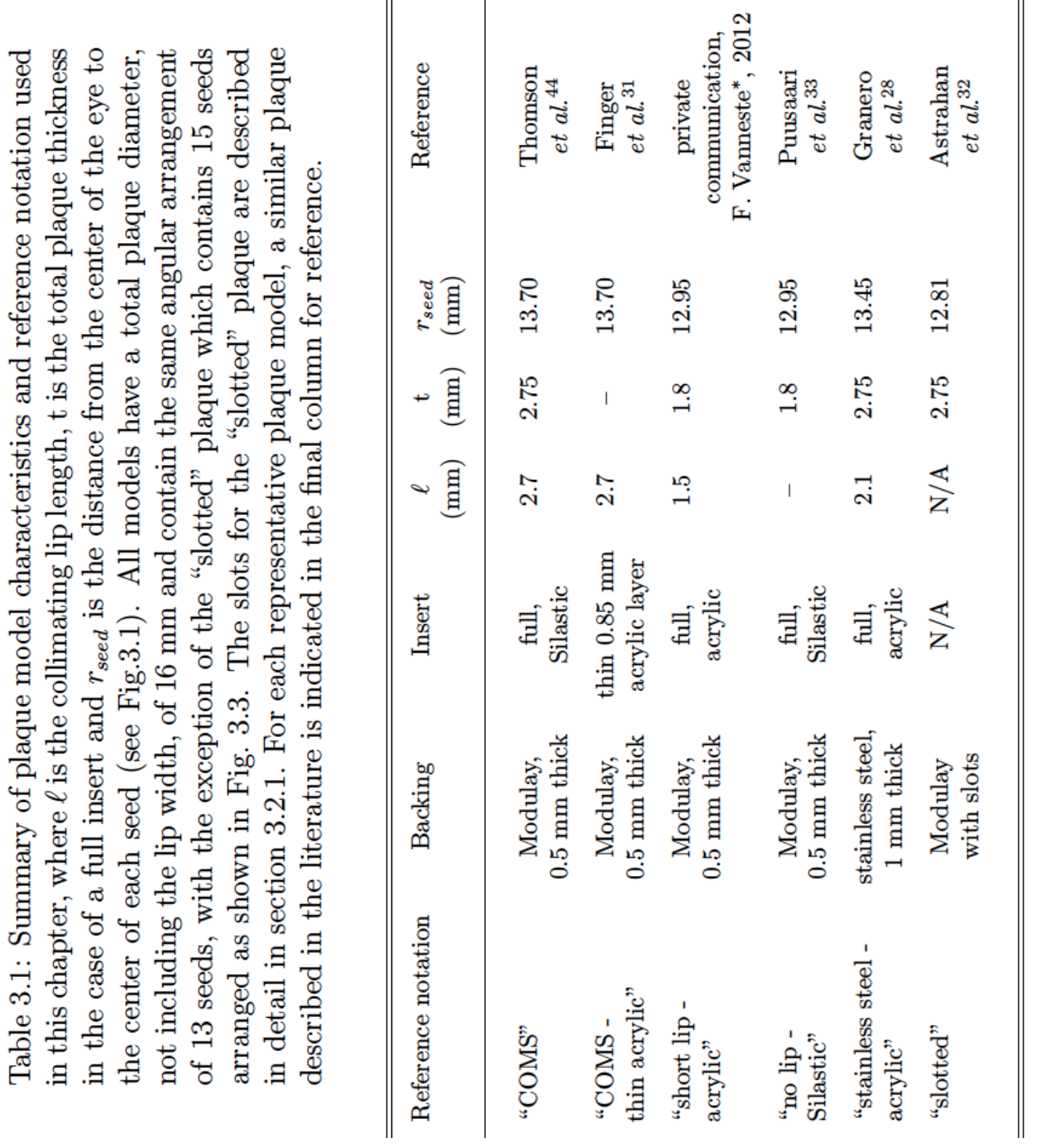




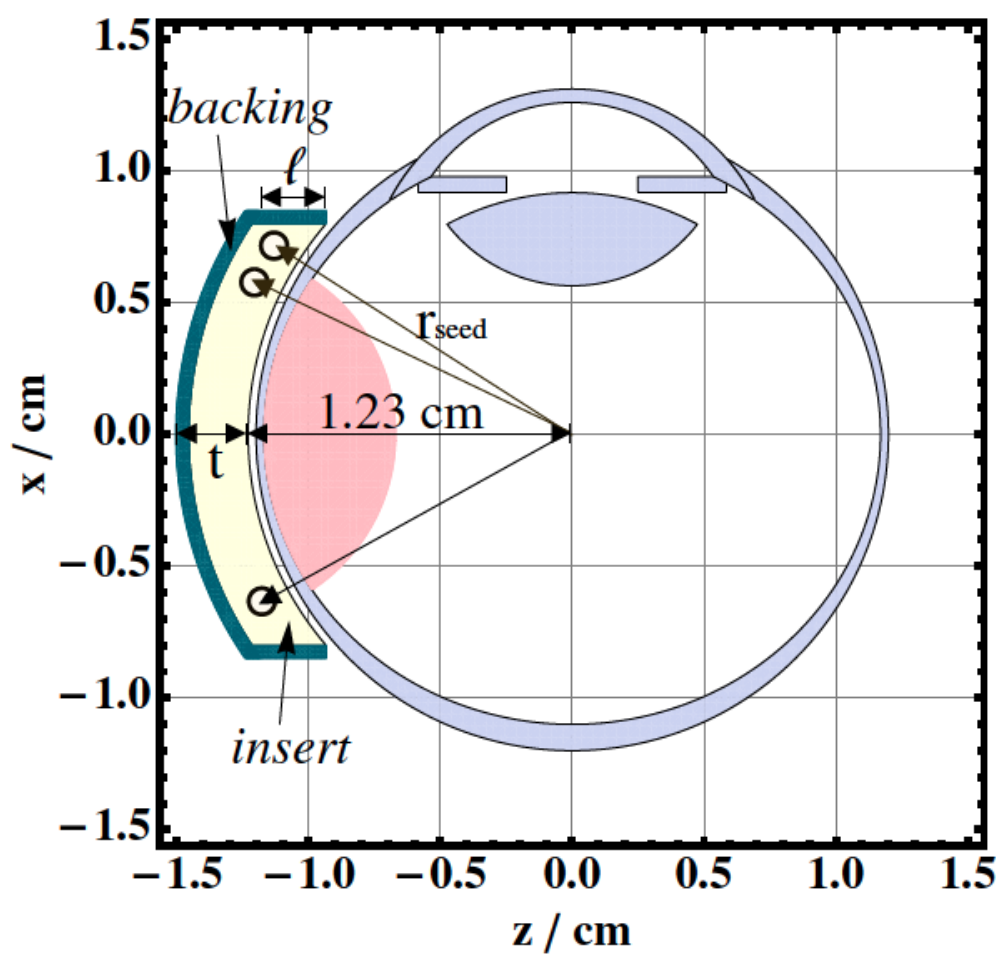

Figure 3.1: Visualization of an eye plaque, with the plaque parameters (used in Table 3.1) indicated, as well as the distance from the center of the eye to the surface of the insert in the case of a full insert $(1.23 \mathrm{~cm})$. A right eye with a nasal-sided tumor is shown in the coordinate system used in this work. 

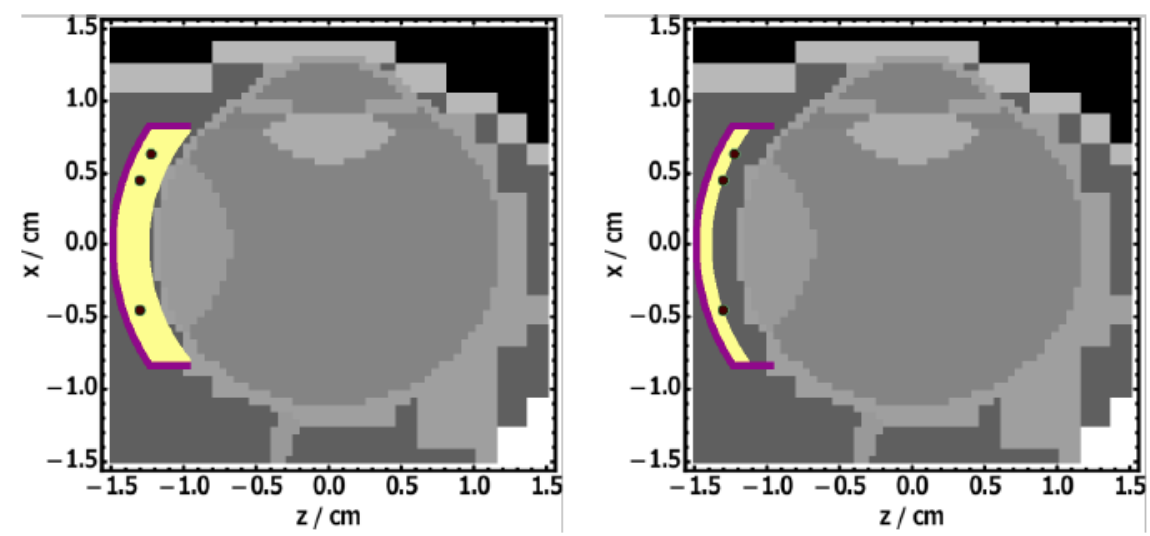

(a) "COMS"

(b) "COMS - thin acrylic"
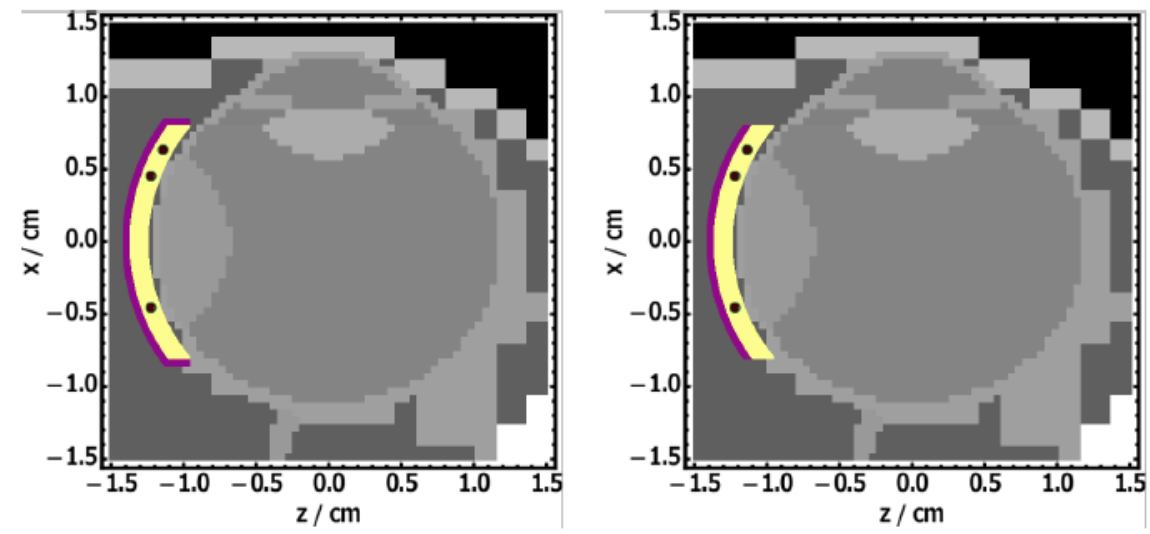

(c) "short lip - acrylic"

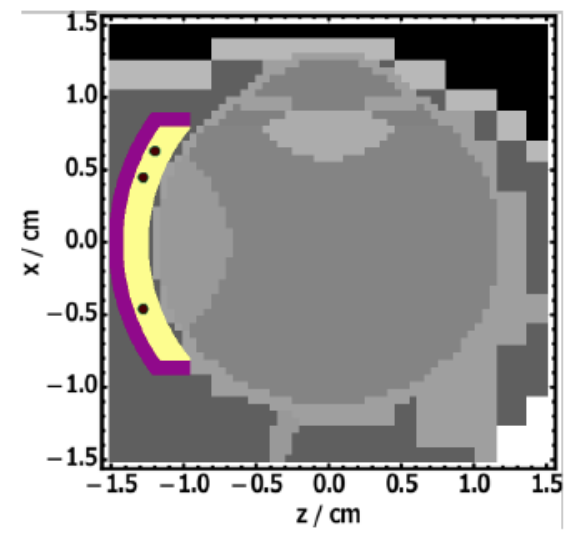

(d) "no lip - Silastic"

(e) "stainless steel - acrylic"

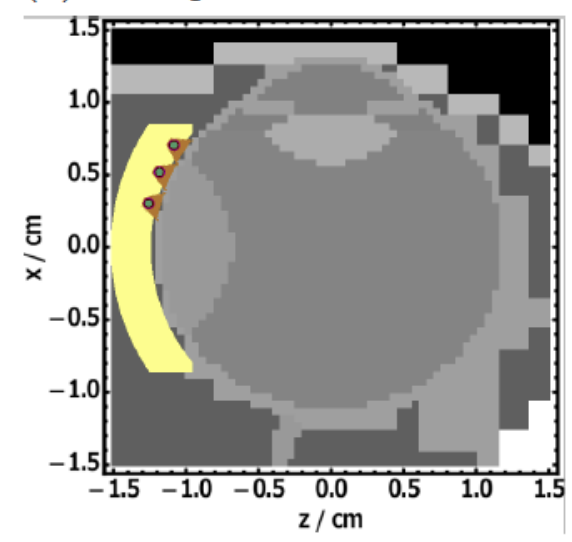

(f) "slotted"

Figure 3.2: Visualization of the representative plaques modeled in this study (described in Table 3.1) with the full eye model. For (a) to (e) the plaque diameter, not including the lip, is $16 \mathrm{~mm}$ and the standard 13-seed arrangement for the regular COMS plaque is used (see TG-129 Report for seed diagram ${ }^{38}$ ); for (f) the seeds are arranged as in Fig. 3.3 and the total plaque diameter is $17 \mathrm{~mm}$ (the same as for the "COMS" plaque when the lip is included in the plaque diameter). The central horizontal slice is shown (voxel centers are at $y=0.025 \mathrm{~cm}$ as voxel boundaries are at $y=0 \mathrm{~cm}$ ). 
The "COMS - thin acrylic" plaque resembles the plaques used by Finger et al., ${ }^{31}$ which have the standard COMS backing and seeds affixed with a thin layer of acrylic. This plaque is also similar to the plaques developed by Astrahan et al. ${ }^{30}$ where a very thin ( $0.3 \mathrm{~mm}$ thick) reusable Modulay seed guide is used to position the seeds in the same seed locations as the COMS plaque, and the seeds are affixed to the backing with an thin layer of acrylic or cyanoacrylate (there is no Silastic insert). The OSU-NAG plaque also resembles the "COMS - thin acrylic" plaque, as it has a Modulay backing with seeds glued directly to the plaque backing, although it uses fewer seeds than the COMS plaque. ${ }^{29}$

The "short lip - acrylic" plaque, with a Modulay backing, an acrylic insert and a $1.5 \mathrm{~mm}$ lip length, is similar to plaques used at the Cliniques Universitaires St-Luc (UCL), Brussels, Belgium. The UCL plaques have lip lengths ranging from 1.3 to 1.5 mm depending on plaque size (private communication, F. Vanneste, 2012), though they differ from the "short lip - acrylic" plaque model with their gold backing and Araldite (an adhesive) insert. Plaques which are thinner and which have shorter lips than the COMS plaque have also been developed by Alberti et al. ${ }^{87}$ although these use a different gold alloy backing with grooves to hold the seeds in place.

The representative plaque without a collimating lip ("no lip - Silastic") resembles the plaques used by Puusaari et al.,$^{33}$ which have a gold backing and a silicone rubber insert. Gold plaques without a lip have also been developed by Nag et al. ${ }^{26}$ but these plaques have seeds affixed to the backing with cyanoacrylate. Although both of these lipless plaques have gold backings, for this study a Modulay backing is used.

The "stainless steel - acrylic" plaque is similar to that documented by Karolis et al. ${ }^{27}$ and the ROPES eye plaque, ${ }^{28}$ which both have a $1 \mathrm{~mm}$ thick backing, a 2.6 $\mathrm{mm}$ (or roughly $3 \mathrm{~mm}$ in the case of the ROPES plaque) overall thickness and a full acrylic insert which holds the seeds in place. ${ }^{27}$ 
Finally, the "slotted" plaque is representative of the plaques developed by Astrahan et al. ${ }^{32}$ and Puusaari et al., ${ }^{32,33}$ which have slots within a gold backing to collimate radiation from each seed. The plaque modeled in this work is composed of Modulay rather than gold and its slots have the same dimensions as those reported by Astrahan et al..$^{32}$ they have a depth of $0.14 \mathrm{~cm}$, a width of $0.06 \mathrm{~cm}$ and a length of $0.46 \mathrm{~cm}$ at the slot's base, and an opening angle of 27 degrees from the normal to the plaque surface in each direction (see Fig. 2 of Astrahan et al..$^{32}$ ).

Although plaques come in different sizes with various seed arrangements, a standard size and seed configuration is chosen for all representative plaque models (with the exception of the "slotted" plaque model) to isolate differences in dose due to the plaque backings, inserts and collimating lips. For all models except the "slotted" plaque, a $16 \mathrm{~mm}$ plaque diameter (excluding the lip width) and the same seed configuration as for the standard $16 \mathrm{~mm}$ COMS plaque with 13 seeds is used (i.e., seeds are placed in three concentric rings of radii: $1.8 \mathrm{~mm}, 4.5 \mathrm{~mm}$, and $6.3 \mathrm{~mm}$ about the $z$ axis $\left.^{38}\right)$; however the distance from the center of the eye to seed centers $\left(r_{\text {seed }}\right)$ varies with plaque thickness (Table 3.1). For the "slotted" plaque, the diameter is $17 \mathrm{~mm}$ (which is the same total diameter, including the lip thickness as for a $16 \mathrm{~mm}$ COMS plaque) and 15 seeds (rather than 13) are modeled (which is the maximum number of seeds for a concentric ring configuration similar to the COMS plaque that does not cause the slots to overlap). More seeds are used in the "slotted" plaque model than in the COMS plaque to approximate the larger number of seeds used in the "slotted" plaques $^{32}$ compared with COMS plaques. ${ }^{38}$ The resulting seed configuration for the "slotted" plaque is shown in Fig. 3.3 and the seed center coordinates for the chosen seed configuration are listed in Table 3.2. All plaque backings and inserts are modeled as segments of spherical shells where the radius of curvature of the concave surface for the full insert (if present) and of the anterior surface in the case of the "slotted" 
plaque is $1.23 \mathrm{~cm}$ (see Fig. 3.1), consistent with the $16 \mathrm{~mm}$ COMS plaque. ${ }^{44}$

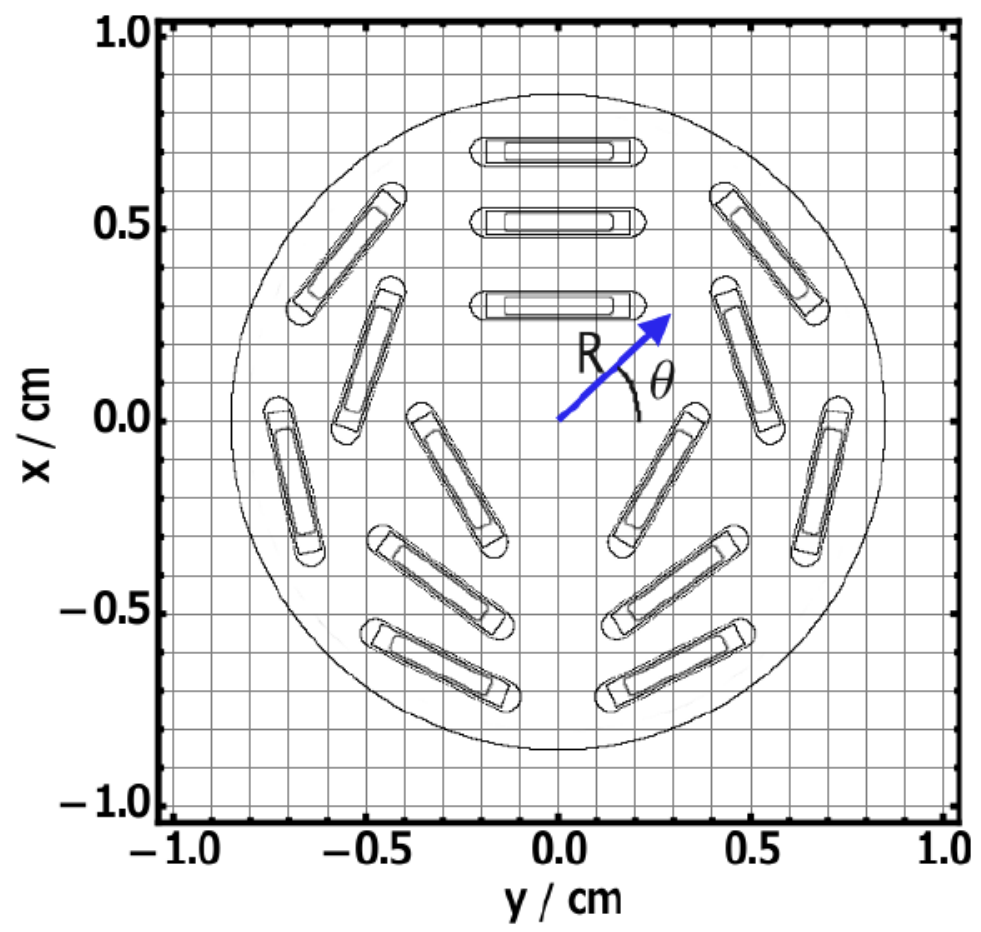

Figure 3.3: Seed diagram for the 15-seed "slotted" $16 \mathrm{~mm}$ Modulay plaque as viewed from the concave aspect of the plaque along its $(z)$ axis; the seeds are shown projected onto the $x y$ plane (in simulations they are placed such that each seed center lies at the same distance from the center of the eye).

The plaque backings for the representative models are either Modulay, which is $77 \%$ gold, $14 \%$ silver, $8 \%$ copper and $1 \%$ palladium by mass ${ }^{44,88}$ and has a density of $15.8 \mathrm{~g} / \mathrm{cm}^{3}$, or stainless steel, which is $71 \%$ iron, $18 \%$ chromium, $9 \%$ nickel, $1 \%$ manganese and $<1 \%$ silicon and carbon by mass and has a density of $8.06 \mathrm{~g} / \mathrm{cm}^{3}$ (composition from the EGSnrc distribution ${ }^{54}$ ). In practice, stainless steel has many possible compositions, therefore another composition (99.05\% iron, $0.0015 \%$ carbon, $0.005 \%$ manganese and $0.003 \%$ silicon by mass with a density of $7.9 \mathrm{~g} / \mathrm{cm}^{3}$, also from the EGSnrc distribution ${ }^{54}$ ) was substituted for the backing of the "stainless steel acrylic" plaque; doses for the plaque in water along the plaque axis agreed within 
Table 3.2: Seed center coordinates for the "slotted" plaque model in the plaque reference frame (see Fig. 3.3).

\begin{tabular}{ccr}
\hline \hline & \multicolumn{2}{c}{$x y$ plane } \\
\cline { 2 - 3 } $\mathrm{z}(\mathrm{cm})$ & $\mathrm{R}(\mathrm{mm})$ & $\theta$ (degrees) \\
-1.245 & 3.01 & 0 \\
& & 210 \\
-1.173 & 5.16 & 330 \\
& & 90 \\
& & 162 \\
& & 234 \\
-1.073 & 7.00 & 216 \\
& & 306 \\
& & 39 \\
& & 90 \\
& & 141 \\
& & 193 \\
& & 244 \\
\hline \hline
\end{tabular}


statistical uncertainties for both compositions. Although various gold backings are used clinically (as mentioned earlier in this section), all plaque models (other than the "stainless steel" plaque) have Modulay backings to provide a consistent comparison between the gold-based plaques. Further, the composition of Modulay is well known unlike many of the gold alloys used in eye plaques. Dosimetric differences between pure gold and Modulay backings have been characterized previously: for a single seed and a water insert, doses for ${ }^{125} \mathrm{I}$ are up to $3 \%$ lower for pure gold compared to Modulay backings along the plaque's central axis, whereas for ${ }^{103} \mathrm{Pd}$ differences between doses along the same axis are negligible for pure gold and Modulay backings. ${ }^{44}$ The inserts for the representative plaque models are either Silastic, which is $39.9 \%$ silicon, $28.9 \%$ oxygen, $24.9 \%$ carbon, $6.3 \%$ hydrogen and $0.005 \%$ platinum by mass and has a density of $1.12 \mathrm{~g} / \mathrm{cm}^{3},{ }^{86}$ or acrylic (i.e., polymethylmethacrylate, or PMMA), which is $60.1 \%$ carbon, $32.0 \%$ oxygen and $8.06 \%$ hydrogen by mass and has a density of $1.19 \mathrm{~g} / \mathrm{cm}^{3}{ }^{89}$ The "slotted" plaque has no insert as it is composed entirely of Modulay.

\subsubsection{Monte Carlo simulations}

Each of the plaque models described in section 3.2.1, as well as the brachytherapy sources $\left({ }^{125} \mathrm{I},{ }^{103} \mathrm{Pd}\right.$, and $\left.{ }^{131} \mathrm{Cs}\right)$ studied in Chapter 2 and the full computational eye model developed in Chapter 2 are fully modeled (i.e., not voxelized), with the exception of discretization of the slots in the "slotted" plaque (Fig. 3.2). Slots within the "slotted" plaque are voxelized based on the slot dimensions described in section 3.2.1 and are filled with water (Fig. 3.2(f)); a resolution of 10 voxels for the entire slot depth is used as a superior resolution had no effect on dose. In practice the slots may fill with blood $;^{90}$ additional simulations with blood-filled slots (rather than water) did not change ocular doses (within statistical uncertainties). Due to the complexity of the model for the "slotted" plaque, each seed position is modeled in a separate simula- 
tion and the resulting doses are added together, ignoring interseed effects; these effects are negligible for this plaque model as seeds are individually collimated (confirmed through additional simulations of multiple slots).

All plaque models are positioned next to the tumor region as shown in Fig. 3.2, such that the insert (in the case of a full insert) is adjacent to the outer sclera of the full eye model. For the "slotted" plaque, the inner edge of the Modulay backing is placed adjacent to the outer sclera. In the case of the "COMS - thin acrylic" plaque, the plaque is placed in the same position as the "COMS" plaque.

In section 3.3.1, results are presented for MC simulations in BrachyDose of each plaque near the center of a $30 \times 30 \times 30 \mathrm{~cm}^{3}$ water phantom containing either a single central seed or fully-loaded (with 13 seeds; 15 for the "slotted" plaque). Dose distributions for the plaque in water $\left(\mathrm{D}_{w, w}\right)$ are compared to those for TG-43 simulations $\left(\mathrm{D}_{T G-43}\right)$ which have the same seed configuration in water but without the plaque or interseed effects. For all plaque models and radionuclides, $\mathrm{D}_{w, w}$ and $\mathrm{D}_{T G-43}$ are normalized such that the tumor apex dose is 85 Gy for the TG-43 simulation. The single seed simulations isolate the effects of the plaque backing/insert on dose; data from these simulations are useful for software such as Plaque Simulator ${ }^{91}$ (which uses dose distributions for a single seed within the eye plaque to calculate multiseed dose distributions for the plaque in a water phantom). The multiseed simulations consider the distribution of seeds within the plaque and are therefore more realistic than single seed simulations. In a subset of simulations, the acrylic insert in the "short lip acrylic" plaque is replaced by a Silastic insert in order to investigate the collimation of radiation in water for three plaques with Modulay backings and Silastic inserts.

In addition to plaque in water $\left(\mathrm{D}_{w, w}\right)$ and TG-43 $\left(\mathrm{D}_{T G-43}\right)$ simulations, fullyloaded plaques are modeled within the computational model of the eye and surrounding tissues developed in Chapter $2\left(\mathrm{D}_{m, m}\right)$. In section 3.3.2, doses for simulations of 
the plaque in the full eye model are compared to those for the plaque in water and to TG-43 using the normalization which gives a $\mathrm{D}_{T G-43}$ of 85 Gy to the tumor apex. In section 3.3.3, doses in the full eye model are presented as percents of the dose to the tumor apex $\left(\% \mathrm{D}_{\text {apex }}\right)$ to provide a direct comparison between each plaque in the full eye model for the three radionuclides studied.

For each simulation in this chapter, $10^{10}$ histories are modeled, resulting in statistical $(1 \sigma)$ dose uncertainties in the full eye model of $0.07-0.1 \%$ at the tumor apex, $0.1-0.2 \%$ at the center of the eye, and $0.3-0.5 \%$ at the opposite side of the sclera to the plaque for all radionuclides within all plaque models. Other sources of uncertainty that are not statistical and which affect the results presented in this chapter are uncertainties in ocular media compositions and eye dimensions (as discussed in Chapter 2), as well as uncertainties in plaque backing and insert compositions and geometry. As mentioned in Chapter 2, there are also uncertainties on interaction cross sections for all media modeled. ${ }^{70}$ Further, the number of seeds and their configuration within the plaques vary by institution (section 3.2.1), which will also affect dose distributions.

\subsection{Results and Discussion}

\subsubsection{Effects of eye plaques on dose distributions in water}

\section{Single seed in eye plaque}

Dose ratios along the plaque, or $z$, axis for the single seed at the center of each plaque to a single seed in water are shown for each radionuclide in Fig. 3.4. For ${ }^{125} \mathrm{I}$ (Fig. 3.4(a)), the largest dose reduction due to the presence of the plaque occurs for the "COMS" plaque; near the plaque (at the inner sclera) $\mathrm{D}_{w, w}$ is roughly $8 \%$ lower than $\mathrm{D}_{T G-43}$, and at the far side of the eye from the plaque $\mathrm{D}_{w, w}$ is $17 \%$ lower than 


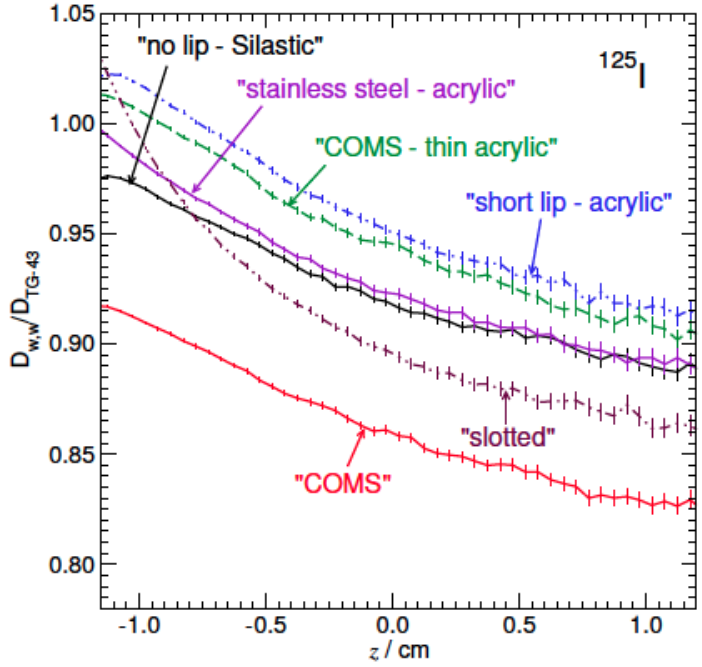

(a)

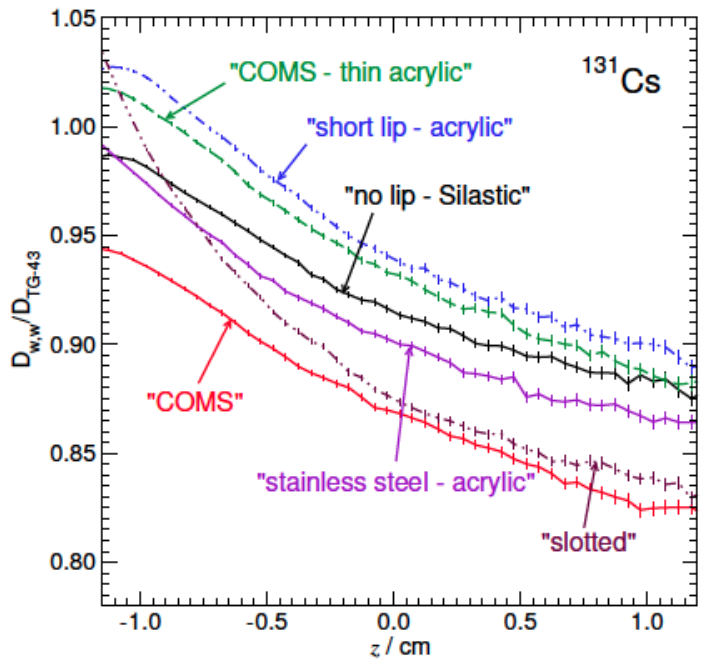

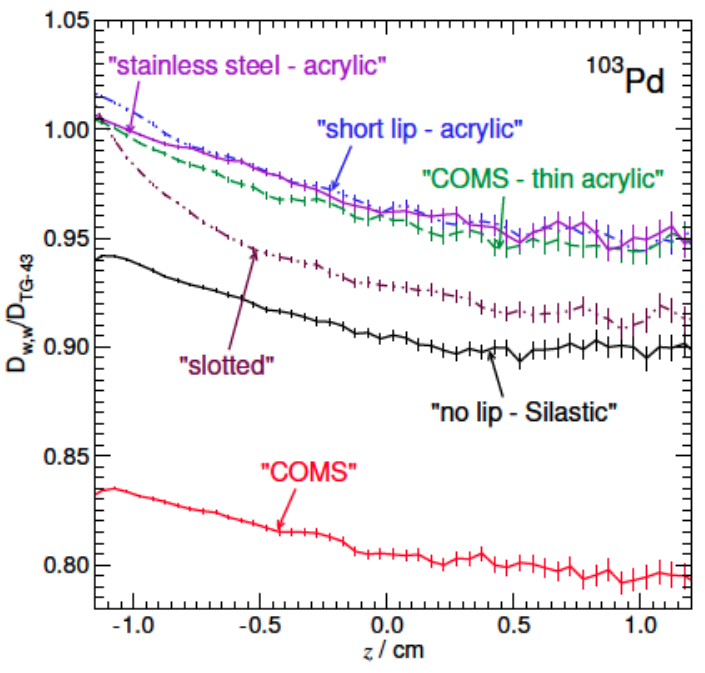

(b)

(c)

Figure 3.4: Dose ratio along the plaque $(z)$ axis for a single seed at the center of each plaque model in water $\left(\mathrm{D}_{w, w}\right)$ to a single seed at the same location in water $\left(\mathrm{D}_{T G-43}\right)$ for (a) ${ }^{125} \mathrm{I}$, (b) ${ }^{103} \mathrm{Pd}$, and (c) ${ }^{131} \mathrm{Cs}$.

$\mathrm{D}_{T G-43}$. When the Silastic insert is replaced with a thin acrylic layer ("COMS - thin acrylic" plaque), $\mathrm{D}_{w, w}$ is larger than $\mathrm{D}_{T G-43}$ by $1 \%$ near the plaque (at the inner sclera) and lower than $\mathrm{D}_{T G-43}$ by $10 \%$ at the opposite side of the eye. The two thin Modulay-backing plaques, "short lip - acrylic" and "no lip - Silastic", show an increase and a decrease of $2 \%$, respectively, near the plaque (at the inner sclera), and decreases of roughly $9 \%$ and $11 \%$, respectively, on the far side of the eye from the plaque for 
$\mathrm{D}_{w, w}$ compared with $\mathrm{D}_{T G-43}$. The "slotted" plaque increases dose compared with $\mathrm{D}_{T G-43}$ by $2.5 \%$ near the plaque (at the inner sclera), and decreases it by $14 \%$ on the opposite side of the eye. The presence of the "stainless steel - acrylic" plaque does not significantly affect dose at the outer sclera (close to the plaque), however at the far side of the eye from the plaque, it reduces the dose compared with the seed alone in water by $11 \%$.

The $\mathrm{D}_{w, w} / \mathrm{D}_{T G-43}$ ratios along the plaque axis for ${ }^{103} \mathrm{Pd}$ and ${ }^{131} \mathrm{Cs}$ (Figs. 3.4(b) and (c), respectively) have less and more of a gradient over distance, respectively, than for ${ }^{125} \mathrm{I}$. For ${ }^{103} \mathrm{Pd}$, the "stainless steel - acrylic", "short lip - acrylic" and "COMS thin acrylic" plaques have similar $\mathrm{D}_{w, w} / \mathrm{D}_{T G-43}$ ratios, with dose enhancements near the plaque (at the inner sclera) of up to $1.5 \%$ and dose reductions at the far side of the eye from the plaque of roughly $5 \%$ for $\mathrm{D}_{w, w}$ compared with $\mathrm{D}_{T G-43}$. For the two plaques with Silastic inserts, "no lip - Silastic" and "COMS", $\mathrm{D}_{w, w}$ is lower than $\mathrm{D}_{T G-43}$ for ${ }^{103} \mathrm{Pd}$ near the plaque (at the inner sclera) by $6 \%$ and $16.5 \%$, respectively, and at the opposite side of the eye by $10 \%$ and $20.5 \%$, respectively. The $\mathrm{D}_{w, w} / \mathrm{D}_{T G-43}$ ratios for ${ }^{131} \mathrm{Cs}$ are within $1 \%$ of those for ${ }^{125} \mathrm{I}$ near the plaque, with the exception of the "COMS" plaque for which there is only a $5.5 \%$ reduction for $\mathrm{D}_{w, w}$ compared with $\mathrm{D}_{T G-43}$ near the plaque (at the inner sclera). At the far side of the eye from the plaque, $\mathrm{D}_{w, w}$ is reduced compared with $\mathrm{D}_{T G-43}$ more for ${ }^{131}$ Cs than for ${ }^{125} \mathrm{I}$ by $1-3 \%$.

For all plaques modeled, the dominant dosimetric effect of the plaque is a reduction in dose due to reduced backscatter from regions behind the plaque. As scattered dose accounts for more of the total dose in regions further from the plaque, a reduction in the number of scattered photons when the plaque is present causes $\mathrm{D}_{w, w} / \mathrm{D}_{T G-43}$ dose ratios to decrease with distance from the plaque. Compton scatter accounts for more interactions in water for ${ }^{125} \mathrm{I}$ and ${ }^{131} \mathrm{Cs}$ than for ${ }^{103} \mathrm{Pd}$, thus dose reductions due to the plaque backing alone (without an insert) are more significant for ${ }^{125} \mathrm{I}$ 
and ${ }^{131} \mathrm{Cs}$ photons than for ${ }^{103} \mathrm{Pd}$ (see Thomson et al. ${ }^{44}$ ). Furthermore, photoelectric interactions in Modulay may produce $25 \mathrm{keV}$ fluorescence photons (among other, lower energy photons) due to the presence of silver (the K-edge of silver is $25 \mathrm{keV}$ ), whereas in stainless steel the fluorescence photons produced are below roughly $8 \mathrm{keV}$ (the highest K-edge in stainless steel is $8.3 \mathrm{keV}$ for Nickel). Since more ${ }^{125} \mathrm{I}$ and ${ }^{131} \mathrm{Cs}$ photons are able to excite the atoms which produce $25 \mathrm{keV}$ fluorescence photons in Modulay than ${ }^{103} \mathrm{Pd}$, differences in dose for Modulay and stainless steel plaques are larger for ${ }^{125} \mathrm{I}$ and ${ }^{131} \mathrm{Cs}$ than for ${ }^{103} \mathrm{Pd}$. Plaques with Silastic cause further reductions in dose due to enhanced photon attenuation in Silastic compared with water, an effect which is larger for ${ }^{103} \mathrm{Pd}$ than for ${ }^{125} \mathrm{I}$ or ${ }^{131} \mathrm{Cs}$ due to its lower average photon energy. Conversely, there is reduced photon attenuation in acrylic compared with water, resulting in increased dose for plaques with an acrylic insert compared with a water insert (confirmed in additional simulations; results not shown).

$\mathrm{D}_{w, w} / \mathrm{D}_{T G-43}$ values for the "COMS" plaque are the lowest of all the plaques for all radionuclides as a result of dose reductions caused by both the plaque backing and insert material (Silastic). For ${ }^{125} \mathrm{I}$ and ${ }^{131} \mathrm{Cs}$, the "slotted" plaque causes the next largest dose reduction in water across most of the eye (for $z>-0.8 \mathrm{~cm}$ ) due to the proximity of Modulay about the seeds and the slot shape (which reduces scatter in the seed's immediate vicinity); near the "slotted" plaque, the ${ }^{125} \mathrm{I}$ and ${ }^{131} \mathrm{Cs}$ dose ratios are higher than for most other plaque models ("COMS", "no lip - Silastic", "stainless steel - acrylic") as a result of fluorescence photons produced in the immediate vicinity of the seeds. All other plaques reduce the dose less than the "COMS" plaque as a result of less photon attenuation in their respective inserts. The $\mathrm{D}_{w, w} / \mathrm{D}_{T G-43}$ ratio for the "stainless steel - acrylic" plaque is lower than for the "short lip - acrylic" plaque (which also has an acrylic insert) for ${ }^{125} \mathrm{I}$ and ${ }^{131} \mathrm{Cs}$ due to the lack of $25 \mathrm{keV}$ fluorescence photons created in the plaque backing compared with Modulay. 


\section{Multiple seeds in eye plaque}

$\mathrm{D}_{w, w} / \mathrm{D}_{T G-43}$ ratios are shown in Fig. 3.5 for fully-loaded (multiseed arrangement) plaques. Dose reductions for fully-loaded plaques for ${ }^{125}$ I range from $2 \%$ for the "short lip - acrylic" plaque to $12 \%$ for the "COMS" plaque at a distance of $5 \mathrm{~mm}$ from the inner sclera (i.e., at the tumor apex at $z=-0.67 \mathrm{~cm}$ ) and from $9.5 \%$ for the "short lip - acrylic" plaque to $18.5 \%$ for the "COMS" plaque at the opposite side of the eye (at the outer sclera). For ${ }^{103} \mathrm{Pd}, \mathrm{D}_{w, w}$ is lower than $\mathrm{D}_{T G-43}$ by $2.5 \%$ ("short lip - acrylic" and "stainless steel - acrylic") to 19.5\% ("COMS") $5 \mathrm{~mm}$ from the inner sclera and by $5 \%$ ("stainless steel - acrylic") to $22 \%$ ("COMS") plaque on the far side

of the eye from the plaque (at the outer sclera). For ${ }^{131} \mathrm{Cs}, \mathrm{D}_{w, w}$ reductions compared with $\mathrm{D}_{T G-43}$ range from $2.5 \%$ ("short lip - acrylic") to $11 \%$ ("COMS") $5 \mathrm{~mm}$ from the inner sclera and from 10.5\% ("short lip - acrylic" and "no lip - Silastic") to $19 \%$ ("COMS") plaque at the opposite side of the eye (at the outer sclera).

$\mathrm{D}_{w, w} / \mathrm{D}_{T G-43}$ ratios for plaques with Silastic inserts ("COMS" and "no lip Silastic") are smaller for multiseed than for single seed simulations along the plaque axis at small $z$ (near the plaque) as photons originating from seeds near the periphery of the plaque pass through more Silastic than those near the center of the plaque to reach points near the plaque along its central axis. The fully-loaded "slotted" plaque also has a substantial reduction in dose near the plaque due to the collimation of the sources. Further from the plaque, differences between $\mathrm{D}_{w, w} / \mathrm{D}_{T G-43}$ values for single seed and fully-loaded plaques are small.

The effect of the lip length on $\mathrm{D}_{w, w} / \mathrm{D}_{T G-43}$ is investigated through additional simulations in which the acrylic insert of the "short lip - acrylic" plaque is replaced by Silastic (i.e., "short lip - Silastic"). The $\mathrm{D}_{w, w} / \mathrm{D}_{T G-43}$ ratio for this plaque is compared with those for the "COMS" and "no lip - Silastic" plaques (which both 


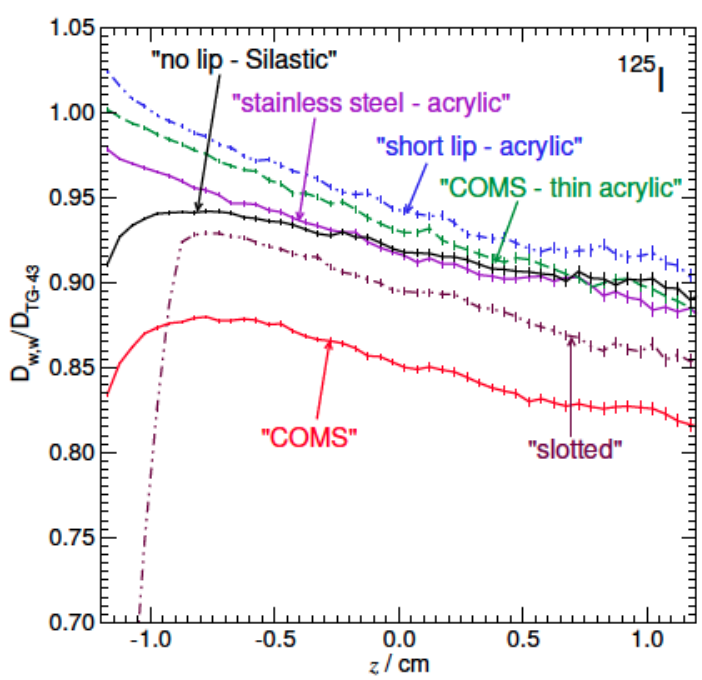

(a)

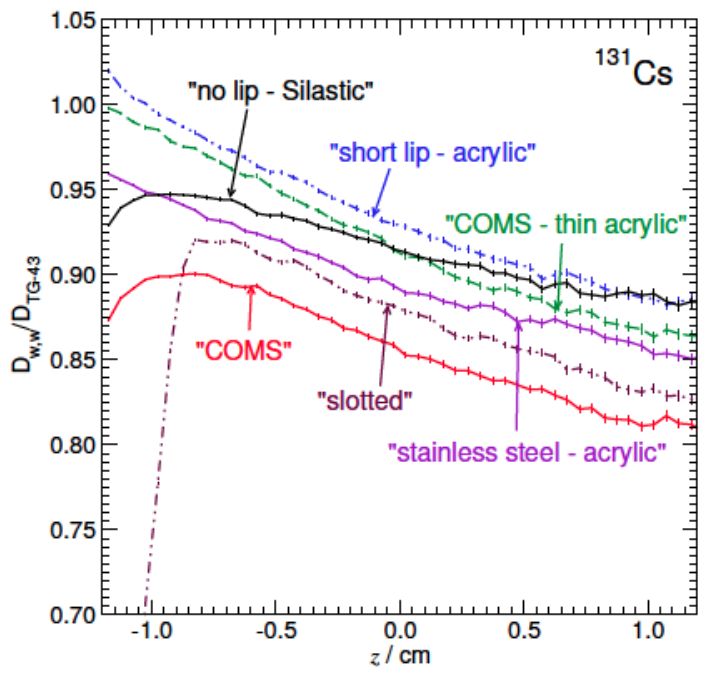

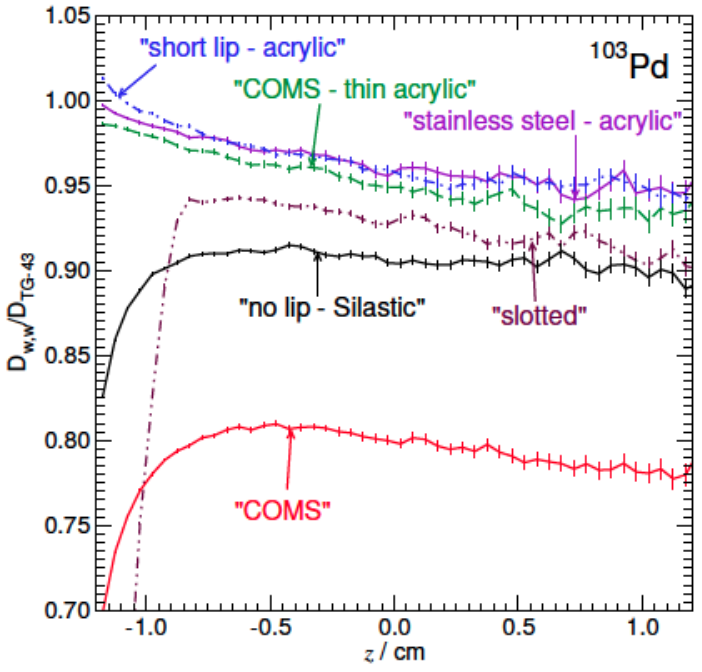

(b)

(c)

Figure 3.5: Dose ratio along the plaque $(z)$ axis for fully-loaded plaques in water $\left(\mathrm{D}_{w, w}\right)$ to the corresponding seed arrangement in water $\left(\mathrm{D}_{T G-43}\right)$ for (a) ${ }^{125} \mathrm{I},(\mathrm{b})$ ${ }^{103} \mathrm{Pd}$, and (c) ${ }^{131} \mathrm{Cs}$.

have Silastic inserts). The $\mathrm{D}_{w, w} / \mathrm{D}_{T G-43}$ ratio along the $z$ axis (Fig. 3.6(a)) shows that dose along the $z$ axis is reduced for longer lips; this dose reduction is due to the additional Silastic between the seeds and the eye when longer lips are used. For the same total plaque thickness, removal of the short lips (i.e., comparing "short lip Silastic" and "no lip - Silastic" plaques) affects $\mathrm{D}_{w, w} / \mathrm{D}_{T G-43}$ values by less than $0.5 \%$ up to the tumor apex $(z=-0.67 \mathrm{~cm})$, however at the far side of the eye from the 


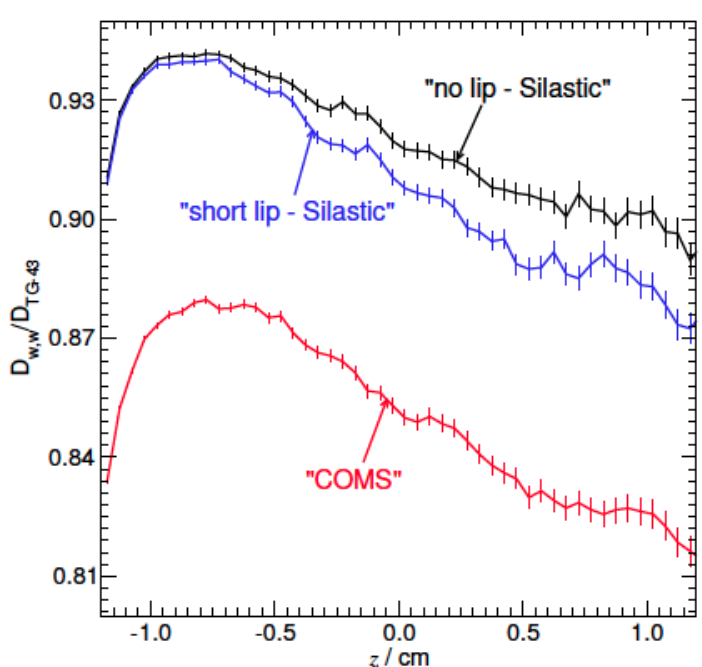

(a)

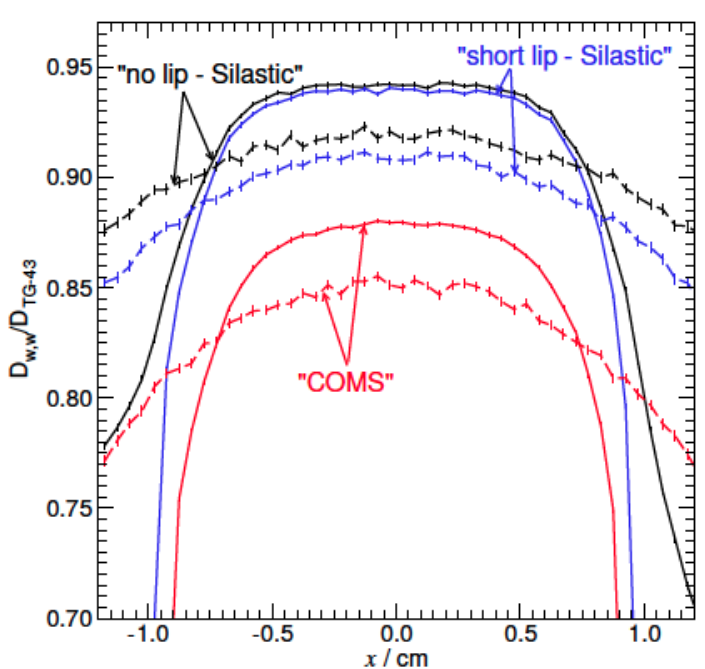

(b)

Figure 3.6: Dose ratio of $\mathrm{D}_{w, w}$ to $\mathrm{D}_{T G-43}$ for ${ }^{125} \mathrm{I}$ (a) along the plaque $(z)$ axis and (b) in the $x$ direction at $z=-0.8 \mathrm{~cm}$ (solid) and $z=0 \mathrm{~cm}$ (dashed) for fully-loaded plaques with Modulay backings and Silastic inserts ("short lip - acrylic" with Silastic insert rather than acrylic, "COMS" and "no lip - Silastic").

plaque, there is a $3 \%$ difference in $\mathrm{D}_{w, w} / \mathrm{D}_{T G-43}$ values as a result of decreased backscatter when the lips are present. The main effect of the lip length/total plaque thickness is on the collimation of photons emitted from sources, demonstrated through comparison of $\mathrm{D}_{w, w} / \mathrm{D}_{T G-43}$ in the $x$ direction for the "COMS", "short lip - Silastic" and "no lip - Silastic" plaques (Fig. 3.6(b)). The $\mathrm{D}_{w, w} / \mathrm{D}_{T G-43}$ ratio drops more steeply when lips are introduced and as lip length increases; this effect is more apparent near the plaque (at $z=-0.8 \mathrm{~cm}$ ) than further from the plaque $($ at $z=0 \mathrm{~cm}$ ).

The effects discussed above regarding collimation contribute to the differences in $\mathrm{D}_{w, w} / \mathrm{D}_{T G-43}$ ratios between the representative plaque models along the $x$ axis (Fig. 3.7). Plaques with shorter lips ("stainless steel - acrylic" and "short lip acrylic") collimate the radiation less (resulting in a smaller decrease in $\mathrm{D}_{w, w} / \mathrm{D}_{T G-43}$ from the center to the periphery of the eye) than those with longer lips ("COMS" and "COMS - thin acrylic"). The variation in $\mathrm{D}_{w, w} / \mathrm{D}_{T G-43}$ between the eye center 
and its periphery is largest for the "slotted" plaque and is smallest for the "no lip Silastic" plaque.

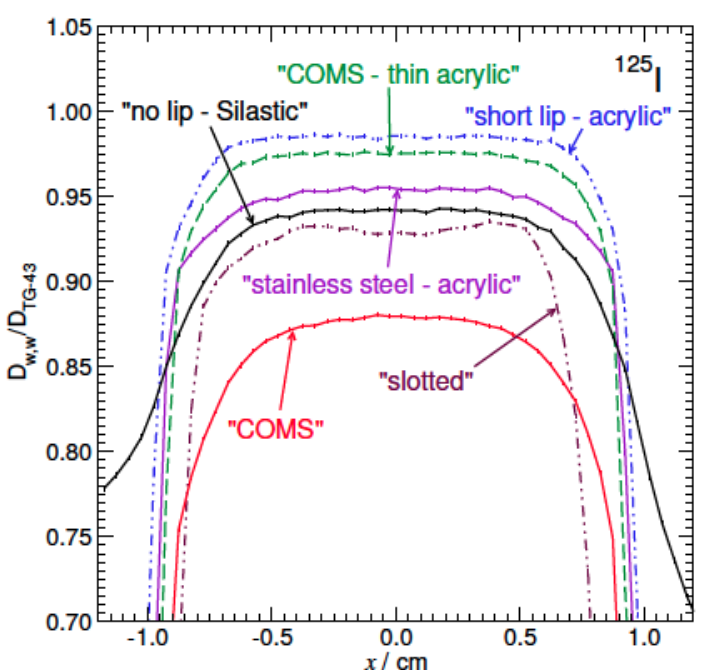

(a)

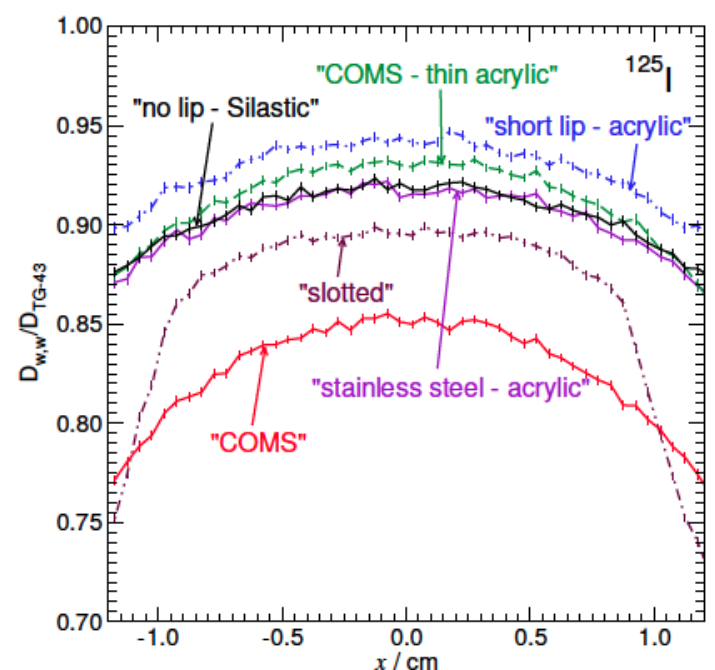

(b)

Figure 3.7: Dose ratio in the $x$ direction at (a) $z=-0.8 \mathrm{~cm}$ (solid) and (b) $z=0$ $\mathrm{cm}$ (dashed) for fully-loaded plaques in water $\left(\mathrm{D}_{w, w}\right)$ to the corresponding seed arrangement in water $\left(\mathrm{D}_{T G-43}\right)$ for ${ }^{125} \mathrm{I}$.

\subsubsection{Dose distributions in the eye; doses to ocular structures}

The effects of ocular media on dose are anticipated given the results presented in Chapter 2 for the $16 \mathrm{~mm}$ COMS plaque in the full eye model: the mass energy absorption and attenuation coefficients of ocular media differ from those for water, affecting dose within the eye significantly. The general effects of both the plaque and ocular media are evident by comparing the ${ }^{125} \mathrm{I}$ isodose contours for $\mathrm{D}_{T G-43}, \mathrm{D}_{w, w}$ and $\mathrm{D}_{m, m}$ (all normalized to a dose of 85 Gy at the apex for the TG-43 simulations) for each of the plaque models (Fig. 3.8). Differences between $\mathrm{D}_{T G-43}, \mathrm{D}_{w, w}$ and $\mathrm{D}_{m, m}$ isodose contours vary with plaque model, with the largest differences throughout the eye volume occurring for the "COMS" and "slotted" plaques and the smallest 
differences occurring for the "short lip - acrylic" plaque. Isodose contours for the "slotted" plaque show an inhomogeneous dose distribution within the tumor, due to the seed arrangement modeled. For all plaques (except the "COMS - thin acrylic" plaque), $\mathrm{D}_{m, m}$ within the tumor volume is closer to $\mathrm{D}_{T G-43}$ than $\mathrm{D}_{w, w}\left(\mathrm{D}_{w, w}\right.$ is lower than both $\mathrm{D}_{m, m}$ and $\mathrm{D}_{T G-43}$ ) for all radionuclides (isodose contours for ${ }^{103} \mathrm{Pd}$ and ${ }^{131} \mathrm{Cs}$ not shown). Within most of the rest of the eye's volume, $\mathrm{D}_{m, m}$ is closer to $\mathrm{D}_{w, w}$ than $\mathrm{D}_{T G-43}$ for all radionuclides $\left(\mathrm{D}_{T G-43}\right.$ is larger than both $\mathrm{D}_{w, w}$ and $\left.\mathrm{D}_{m, m}\right)$; for the "short lip - acrylic", "stainless steel - acrylic" and "COMS - thin acrylic" plaques, dose is higher for the $\mathrm{D}_{m, m}$ than $\mathrm{D}_{T G-43}$ in regions of the vitreous close to the plaque.

Maximum, minimum and average doses to ocular volumes of interest within the eye are presented for the TG-43 simulations $\left(\mathrm{D}_{T G-43}\right)$, and simulations of the plaque in water $\left(\mathrm{D}_{w, w}\right)$ and in the full eye model $\left(\mathrm{D}_{m, m}\right)$ in Tables 3.3 to 3.5 and are discussed in this section. 

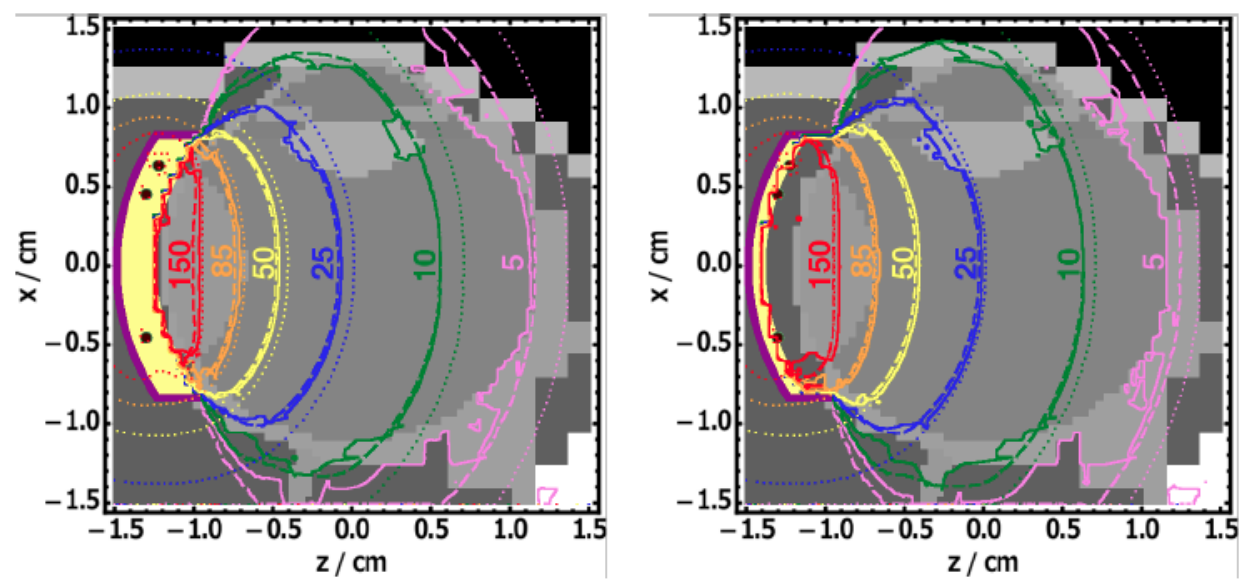

(a) "COMS"

(b) "COMS - thin acrylic"
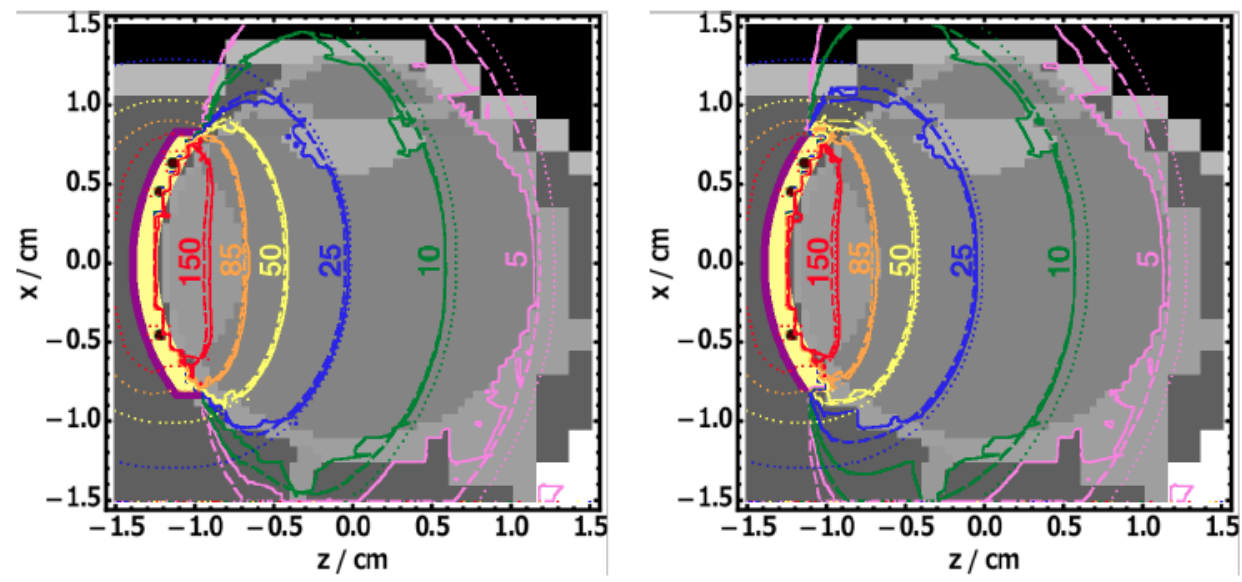

(c) "short lip - acrylic"

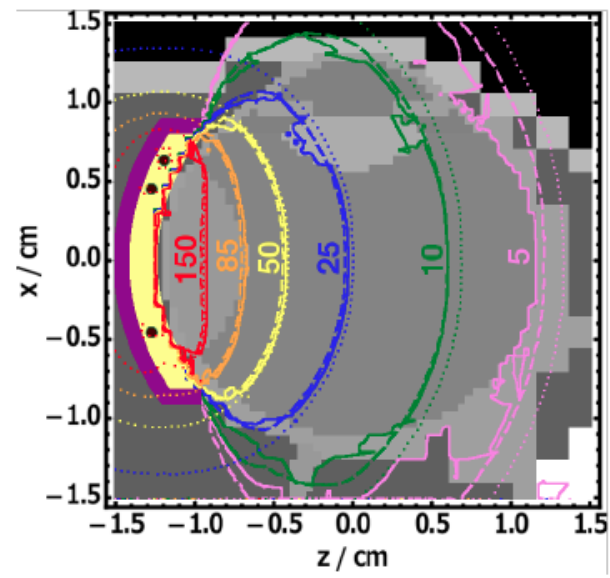

(d) "no lip - Silastic"

(e) "stainless steel - acrylic"

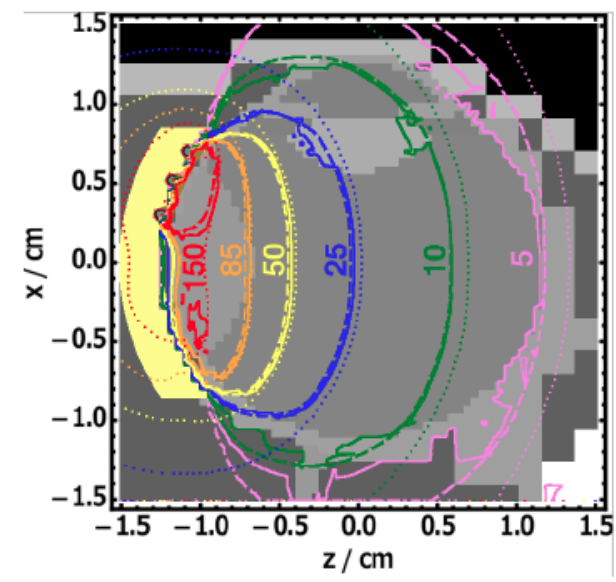

(f) "slotted"

Figure 3.8: ${ }^{125} \mathrm{I}$ isodose contours for the central horizontal plane (voxel centers are at $y=0.025 \mathrm{~cm}$ as voxel boundaries are at $y=0 \mathrm{~cm})$ from $\mathrm{D}_{T G-43}$ (dotted), $\mathrm{D}_{w, w}$ (dashed), and $\mathrm{D}_{m, m}$ (solid) simulations for each plaque model. Doses are normalized to $85 \mathrm{~Gy}$ at the tumor apex in the TG-43 simulation. Although dose is not scored within the plaque, isodose contours intersect the plaque in voxels which are partially occupied by the plaque with centers lying within the plaque. 


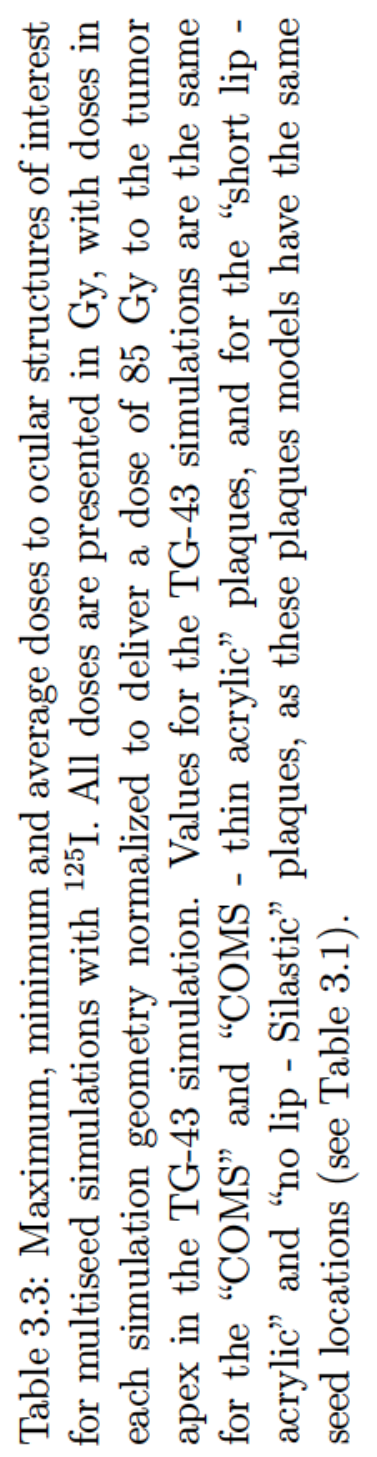

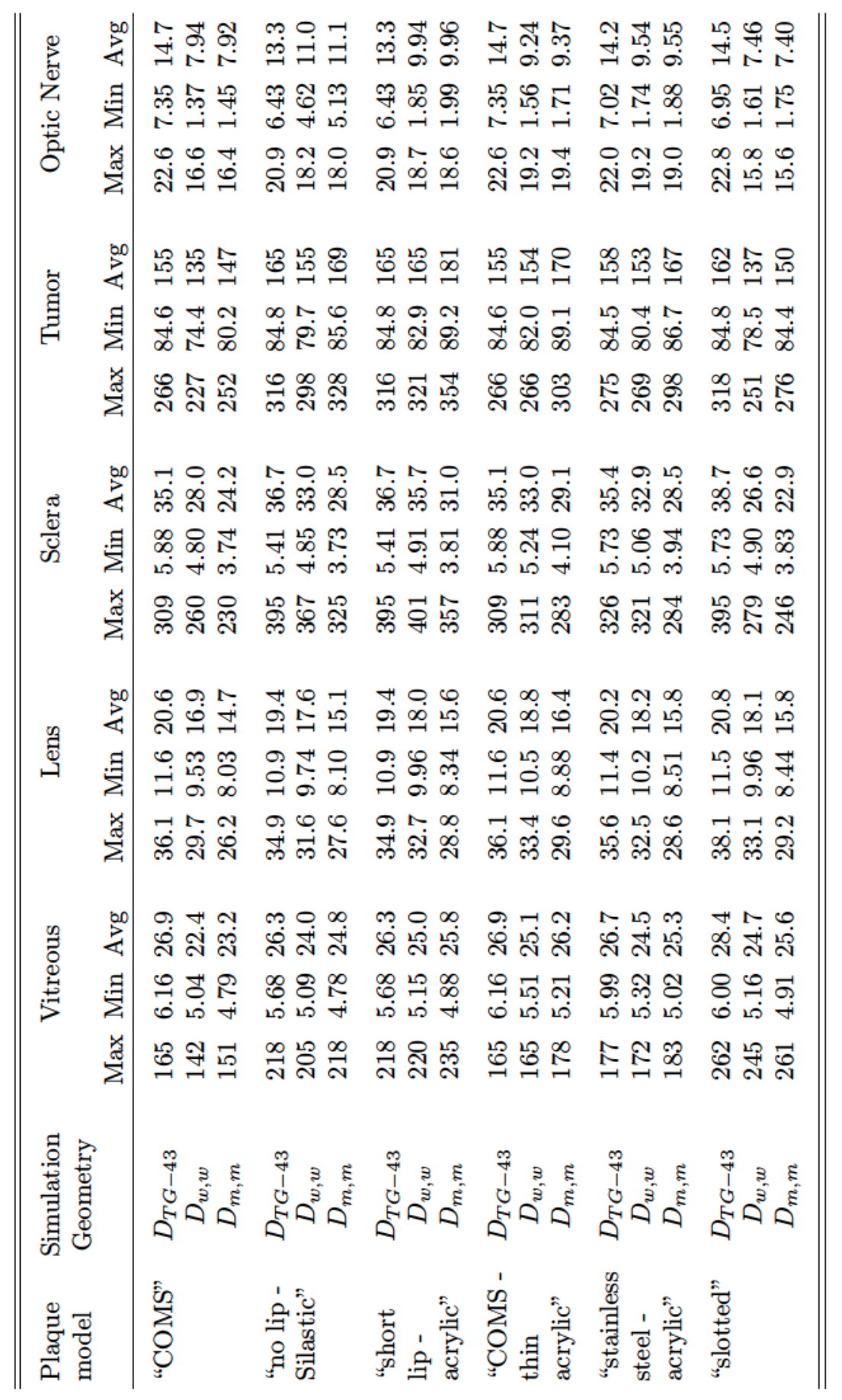




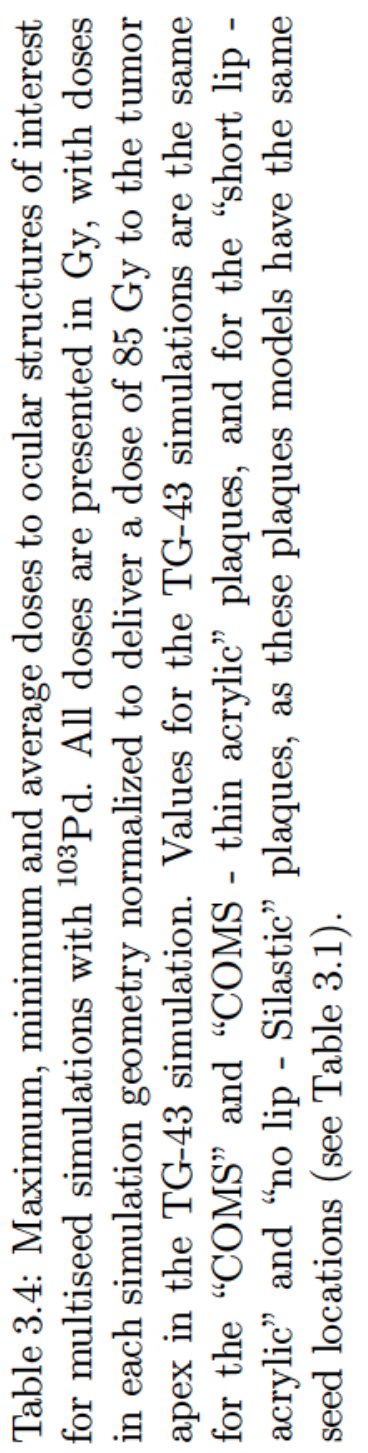

\begin{tabular}{|c|c|c|c|c|c|c|}
\hline$\sum_{0}^{\infty} \quad \frac{\infty}{4}$ & 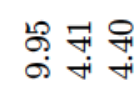 & 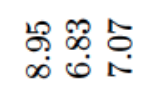 & 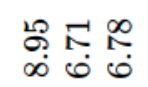 & 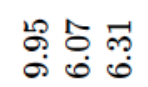 & 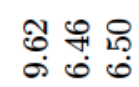 & 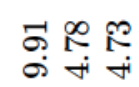 \\
\hline 妾 & 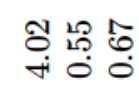 & 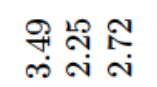 & 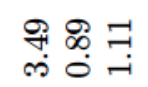 & 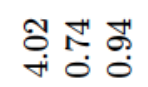 & 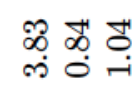 & 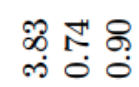 \\
\hline 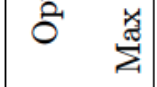 & 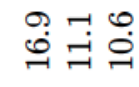 & 通 & 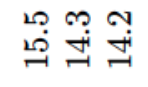 & 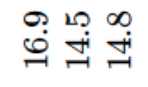 & 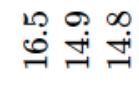 & 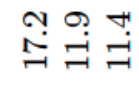 \\
\hline$\sum_{4}^{\infty}$ & : & 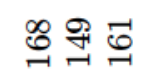 & 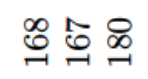 & 象员 & 엄 & 屏年 \\
\hline 妾 & 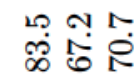 & 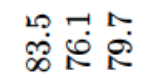 & 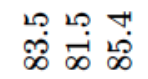 & $\begin{array}{l}20 \\
\infty \\
\infty \\
\infty\end{array}$ & $\begin{array}{l}0 \\
\dot{B} \\
\infty\end{array}$ & $\begin{array}{l}\wedge \\
\infty \\
\infty\end{array}$ \\
\hline 囦 & ఖ్టి & $\vec{m} \stackrel{2}{\stackrel{2}{N} \vec{~}}$ & $\vec{m} \stackrel{m}{m} \underset{m}{m}$ & 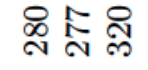 & 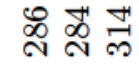 & స్ల స్ స్ స్తే \\
\hline 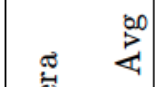 & 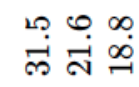 & 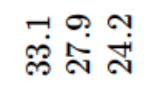 & 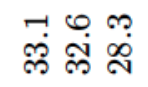 & 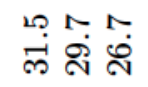 & 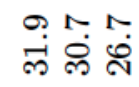 & 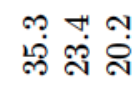 \\
\hline 竞 & 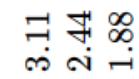 & 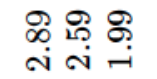 & 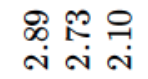 & 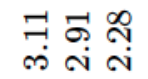 & 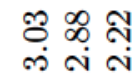 & 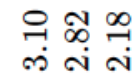 \\
\hline 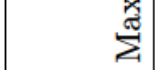 & స్లి సి సి & 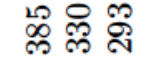 & 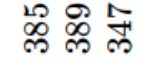 & $\overrightarrow{\widetilde{N}} \widetilde{\sim}$ & స్లి స్లి స్ స్ & స్లో \\
\hline$\stackrel{\infty}{\frac{\infty}{4}}$ & 刍 & r & 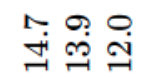 & & กี & 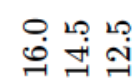 \\
\hline 䍃 & 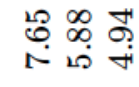 & 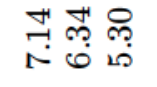 & 苞足 & 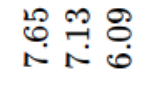 & 市 & 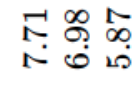 \\
\hline 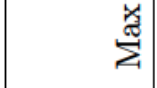 & 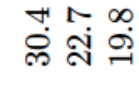 & 茫 & ه্র & 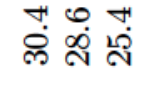 & 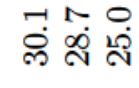 & 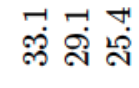 \\
\hline$\stackrel{\infty}{\frac{\infty}{4}}$ & 茫号莣 & 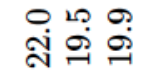 & 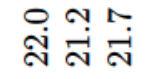 & 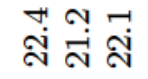 & กี่ & 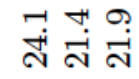 \\
\hline$\stackrel{\Xi}{\Sigma}$ & 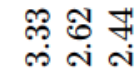 & 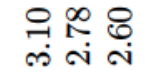 & 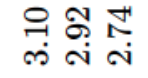 & 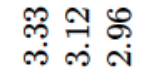 & 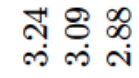 & 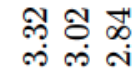 \\
\hline 党 & 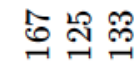 & 웡 & : ڤి & 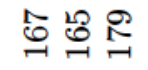 & 点点学 & ฝึ \\
\hline 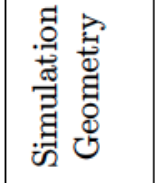 & 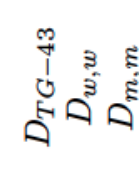 & & & & & 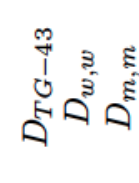 \\
\hline 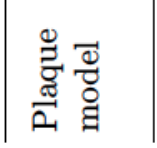 & 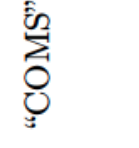 & 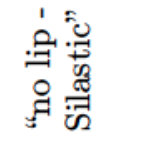 & 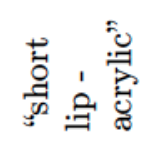 & 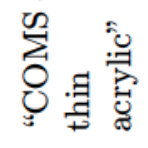 & 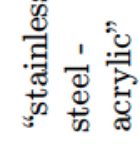 & 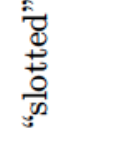 \\
\hline
\end{tabular}




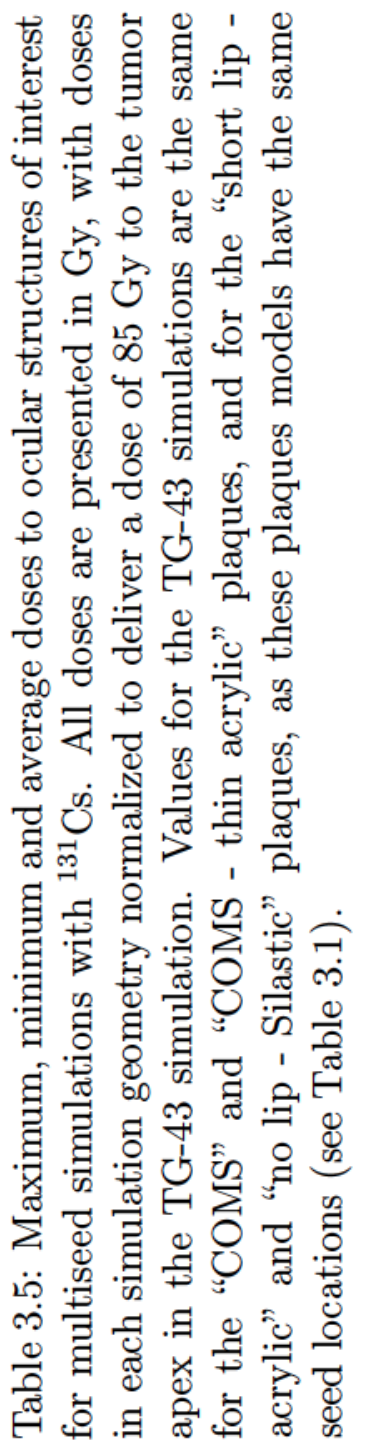

\begin{tabular}{|c|c|c|c|c|c|c|}
\hline$\stackrel{0}{4}_{4}^{\infty}$ & 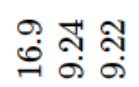 & 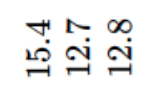 & 苞 & 象 & 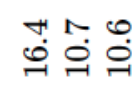 & 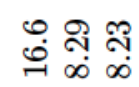 \\
\hline 妾 & 占志界 & 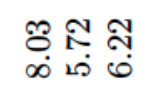 & 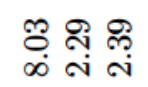 & 요용 & 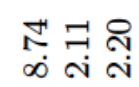 & 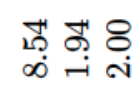 \\
\hline$\stackrel{:}{\pi}$ & 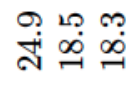 & 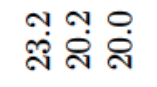 & 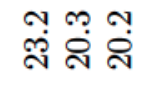 & 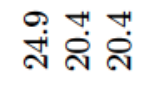 & ثี & 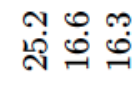 \\
\hline 点 & 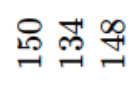 & 品焉总 & 品哭哭 & 员 $\underset{\sim}{\stackrel{\infty}{\rightarrow ~}}$ & 骂哭适 & 呁品㴔 \\
\hline 塄 & 诂苫 & 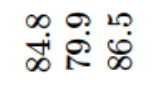 & $\begin{array}{l}\infty \\
\dot{\infty} \\
\dot{\infty}\end{array}$ & 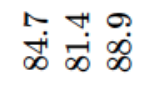 & \begin{tabular}{l}
$\infty$ \\
\multirow{i}{*}{$\infty$} \\
$\infty$
\end{tabular} & 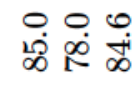 \\
\hline 茪 & 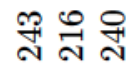 & 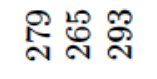 & 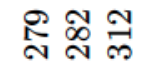 & 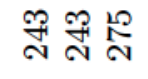 & 品 융 商 & 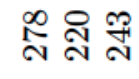 \\
\hline 点 & $\begin{array}{l}\text { m } \\
\dot{\varphi}\end{array}$ & 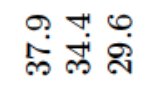 & 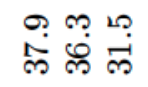 & 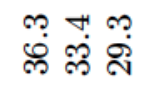 & 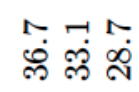 & 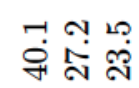 \\
\hline 竞 & 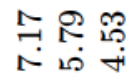 & 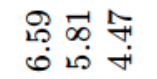 & 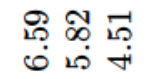 & 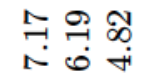 & 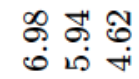 & 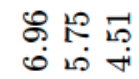 \\
\hline 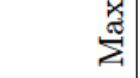 & 온 & 㟧 & బొ๊ & ํ) 논 & \& & 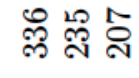 \\
\hline$\stackrel{\infty}{4}^{\infty}$ & 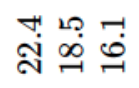 & ڤُ & 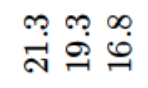 & สี่ & 옴 & ف웜 \\
\hline 䍃 & 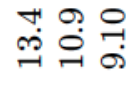 & 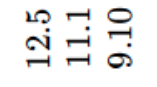 & 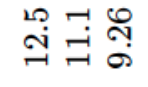 & 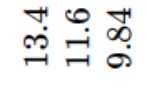 & 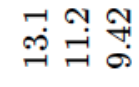 & 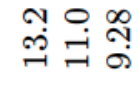 \\
\hline 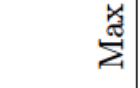 & 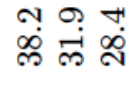 & 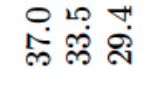 & 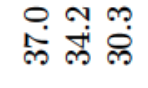 & 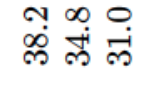 & 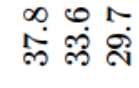 & 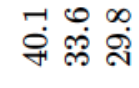 \\
\hline$\stackrel{5}{4}$ & 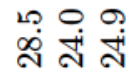 & 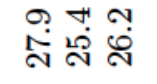 & 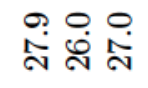 & 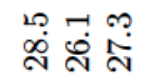 & 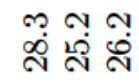 & 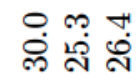 \\
\hline 声 & 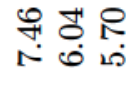 & 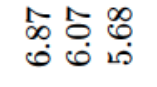 & 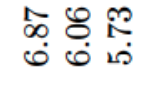 & 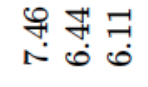 & 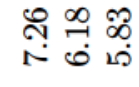 & 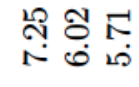 \\
\hline 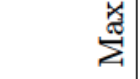 & 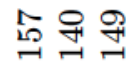 & 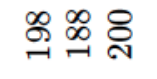 & 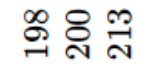 & 占员虽曽 & & ๓ึ \\
\hline 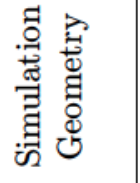 & 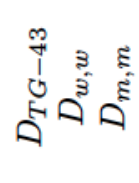 & & & & & 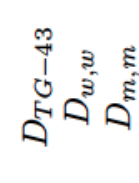 \\
\hline 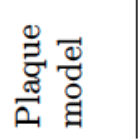 & $\sum_{0}^{20}$ & 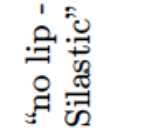 & 苛: & 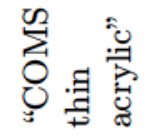 & 苞 & 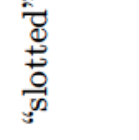 \\
\hline
\end{tabular}




\section{Tumor}

Differences between the average tumor $\mathrm{D}_{m, m}$ and $\mathrm{D}_{T G-43}$ (Tables 3.3 to 3.5 ) vary between the plaque models, with $\mathrm{D}_{m, m}$ up to $8 \%$ lower than $\mathrm{D}_{T G-43}$ ("slotted") and up to $10 \%$ higher than $\mathrm{D}_{T G-43}$ ("short lip - acrylic" and "COMS - thin acrylic") for ${ }^{125} \mathrm{I}$. For ${ }^{103} \mathrm{Pd}, \mathrm{D}_{m, m}$ is up to $17 \%$ lower than $\mathrm{D}_{T G-43}$ ("COMS") and up to $8 \%$ higher than $\mathrm{D}_{T G-43}$ ("COMS - thin acrylic"). For ${ }^{131} \mathrm{Cs}, \mathrm{D}_{m, m}$ is up to $9 \%$ lower than $\mathrm{D}_{T G-43}$ ("slotted") and up to $10 \%$ higher than $\mathrm{D}_{T G-43}$ ("short lip - acrylic"). These discrepancies between $\mathrm{D}_{T G-43}$ and $\mathrm{D}_{m, m}$ are caused by the combined effects of the plaque (section 3.3.1) and the higher mass energy absorption and attenuation coefficients in tumor tissue than in water (discussed in Chapter 2).

\section{Lens}

For all plaque models, dose in the lens is reduced in the full eye model compared to $\mathrm{D}_{T G-43}$ (Tables 3.3 to 3.5 ) due to plaque effects, reduced photon fluence (resulting from radiation transport through the tumor and vitreous) and a lower mass energy absorption coefficient in lens material than in water (Chapter 2). The maximum $\mathrm{D}_{m, m}$ received by the lens is lower than the maximum $\mathrm{D}_{T G-43}$ by $17 \%$ ("short lip - acrylic") up to $27 \%$ ("COMS") for ${ }^{125} \mathrm{I}$, by $17 \%$ ("short lip - acrylic", "COMS - thin acrylic" and "stainless steel - acrylic") to $35 \%$ ("COMS") for ${ }^{103} \mathrm{Pd}$, and by $18 \%$ ("short lip acrylic") up to $26 \%$ ("COMS" and "slotted") for ${ }^{131}$ Cs. The corresponding percent differences are slightly larger for the average lens dose, ranging from $20-29 \%$ for ${ }^{125} \mathrm{I}$, from $18-35 \%$ for ${ }^{103} \mathrm{Pd}$, and from $21-28 \%$ for ${ }^{131} \mathrm{Cs}$. 


\section{Vitreous}

Average vitreous $\mathrm{D}_{m, m}$ is lower than $\mathrm{D}_{T G-43}$ for all plaque models (Tables 3.3 to 3.5) by $2-14 \%$ for ${ }^{125} \mathrm{I}$, by $1-22 \%$ for ${ }^{103} \mathrm{Pd}$, and by $3-12 \%$ for ${ }^{131} \mathrm{Cs}$. In all cases, the largest difference between $\mathrm{D}_{m, m}$ and $\mathrm{D}_{T G-43}$ occurs for the "COMS" plaque and the smallest for the "short lip - acrylic" plaque (for ${ }^{103} \mathrm{Pd}$, the "COMS - thin acrylic" has a similar reduction to the "short lip - acrylic", and for ${ }^{131}$ Cs the "slotted" plaque has a similar reduction to the "COMS" plaque). The minimum $\mathrm{D}_{m, m}$ is lower than $\mathrm{D}_{T G-43}$ for all plaque models. For some plaque models and radionuclides, maximum vitreous $\mathrm{D}_{m, m}$ is higher than $\mathrm{D}_{T G-43}$, whereas for others it is lower. As discussed in Chapter 2, the dose in the vitreous of the full eye model $\left(D_{m, m}\right)$ is higher near the plaque (compared to in the $\mathrm{D}_{w, w}$ simulation) due to its higher mass energy absorption coefficient than in water; at the opposite side of the eye, a decrease in $\mathrm{D}_{m, m}$ in the vitreous compared with $\mathrm{D}_{w, w}$ is observed due to more photon attenuation in the vitreous compared with in water.

\section{Sclera}

$\mathrm{D}_{m, m}$ to the sclera is lower than $\mathrm{D}_{T G-43}$ for all plaque models (Tables 3.3 to 3.5 ); for the maximum scleral dose these reductions range from $8-38 \%$ for ${ }^{125} \mathrm{I}$, from $7-39 \%$ for ${ }^{103} \mathrm{Pd}$, and from $9-38 \%$ for ${ }^{131} \mathrm{Cs}$, with the smallest reductions for the "COMS - thin acrylic" plaque and the largest for the "slotted" plaque for each radionuclide. Scleral doses (maximum, minimum and average) are reduced as a result of the lower mass energy absorption coefficient for sclera than water, plaque effects, and increased photon attenuation through the tumor and vitreous volumes (which mainly affects scleral regions far from the plaque). The "slotted" plaque has the largest maximum and average $\mathrm{D}_{m, m}$ scleral dose reductions compared with $\mathrm{D}_{T G-43}$ due to the collimating 
slots about seeds.

\section{Optic Nerve}

$\mathrm{D}_{m, m}$ to the optic nerve is considerably different than $\mathrm{D}_{T G-43}$ to the same region (Tables 3.3 to 3.5 ). For ${ }^{125} \mathrm{I}$, the maximum $\mathrm{D}_{m, m}$ to the optic nerve is lower than $\mathrm{D}_{T G-43}$ by $11 \%$ ("short lip - acrylic") to $32 \%$ ("slotted"), by $9 \%$ ("short lip - acrylic") to $37 \%$ ("COMS") for ${ }^{103} \mathrm{Pd}$, and by $13 \%$ ("short lip - acrylic") to $35 \%$ ("slotted") for ${ }^{131} \mathrm{Cs}$. The optic nerve has a mass energy absorption coefficient near that of water causing optic nerve $\mathrm{D}_{m, m}$ and $\mathrm{D}_{w, w}$ values in Tables 3.3 to 3.5 to nearly agree. This suggests that discrepancies between $\mathrm{D}_{m, m}$ and $\mathrm{D}_{T G-43}$ to this structure are due mostly to plaque effects and differences in attenuation and scatter through ocular media other than the optic nerve.

\subsubsection{Comparison of plaque models within the full eye model}

Doses for this section are presented in terms of the percentage of the dose to the tumor apex $\left(\% \mathrm{D}_{\text {apex }}\right)$ in the full eye model for each plaque model and radionuclide (Table 3.6, Fig. 3.9).

For each radionuclide, the largest average $\% \mathrm{D}_{\text {apex }}$ to the tumor is delivered by the "short lip - acrylic" plaque $\left(202 \% \mathrm{D}_{\text {apex }}\right.$ for ${ }^{125} \mathrm{I}, 207 \% \mathrm{D}_{\text {apex }}$ for ${ }^{103} \mathrm{Pd}$, and $195 \% \mathrm{D}_{\text {apex }}$ for $\left.{ }^{131} \mathrm{Cs}\right)$, and the smallest by the "slotted" plaque $\left(177 \% \mathrm{D}_{\text {apex }}\right.$ for ${ }^{125} \mathrm{I}, 179 \% \mathrm{D}_{\text {apex }}$

for ${ }^{103} \mathrm{Pd}$, and $169 \% \mathrm{D}_{\text {apex }}$ for ${ }^{131} \mathrm{Cs}$ ). Between plaque models (loaded with seeds of the same radionuclide), average vitreous doses vary little (by $2 \% \mathrm{D}_{\text {apex }}$ ); however, maximum vitreous doses vary considerably (with ranges of $121 \% \mathrm{D}_{\text {apex }}$ for ${ }^{125} \mathrm{I}, 94$ $\% \mathrm{D}_{\text {apex }}$ for ${ }^{103} \mathrm{Pd}$ and $86 \% \mathrm{D}_{\text {apex }}$ for $\left.{ }^{131} \mathrm{Cs}\right)$. For each radionuclide, the "COMS" plaque delivers the lowest maximum vitreous dose and the "slotted" eye plaque the 
highest. The maximum $\% \mathrm{D}_{\text {apex }}$ values to the lens for all plaque models (loaded with seeds of the same radionuclide) are within $1 \% \mathrm{D}_{\text {apex }}$ of each other for ${ }^{131} \mathrm{Cs}$, and within $3 \% \mathrm{D}_{\text {apex }}$ of each other for ${ }^{125} \mathrm{I}$ and ${ }^{103} \mathrm{Pd}$; average lens $\% \mathrm{D}_{\text {apex }}$ values for all plaque models are within $2 \% \mathrm{D}_{\text {apex }}$ for each radionuclide. The lowest maximum $\% \mathrm{D}_{\text {apex }}$ to the lens is delivered by the "no lip - Silastic" and "short lip - acrylic" plaques for ${ }^{125} \mathrm{I}$, and by the "COMS" plaque for ${ }^{103} \mathrm{Pd}$; four of the six plaque models give the same maximum lens $\% \mathrm{D}_{\text {apex }}$ for ${ }^{131} \mathrm{Cs}$. The maximum scleral $\% \mathrm{D}_{\text {apex }}$ varies widely between plaque models for each radionuclide, with the lowest value for the "COMS" plaque for ${ }^{125} \mathrm{I}$, and for the "slotted" plaque for ${ }^{103} \mathrm{Pd}$ and ${ }^{131} \mathrm{Cs}$; the highest maximum scleral $\% \mathrm{D}_{\text {apex }}$ occurs for the "short lip - acrylic" plaque for each radionuclide. Finally, the maximum $\% \mathrm{D}_{\text {apex }}$ to the optic nerve is highest for the "stainless steel - acrylic" and "COMS - thin acrylic" plaques and lowest for the "slotted" plaque for each radionuclide (for ${ }^{131} \mathrm{Cs}$, the "no lip - Silastic" and "short lip - acrylic" plaques deliver the same maximum $\% \mathrm{D}_{\text {apex }}$ to the optic nerve).

Comparing the radionuclides, the average tumor $\% \mathrm{D}_{\text {apex }}$ (Table 3.6) is lowest for ${ }^{131} \mathrm{Cs}$ and highest for ${ }^{103} \mathrm{Pd}$ for all plaques; for all other ocular structures listed in Table 3.6, the average $\% \mathrm{D}_{\text {apex }}$ for each plaque is lowest for ${ }^{103} \mathrm{Pd}$ and highest for ${ }^{131} \mathrm{Cs}$. For the minimum and maximum $\% \mathrm{D}_{\text {apex }}$ to all ocular structures, the radionuclide with the highest and lowest $\% \mathrm{D}_{\text {apex }}$ varies between plaques. 


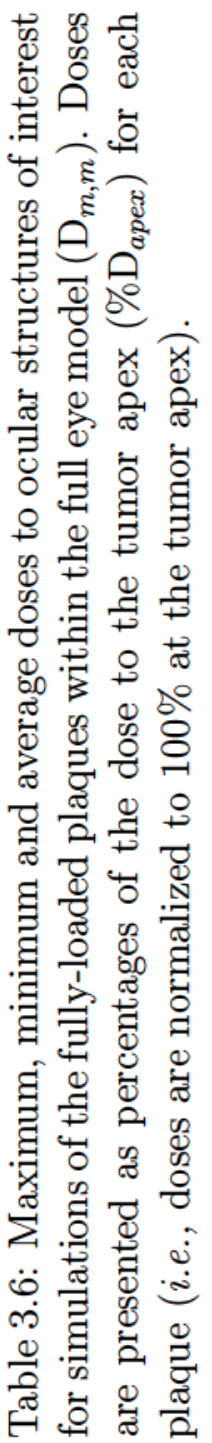

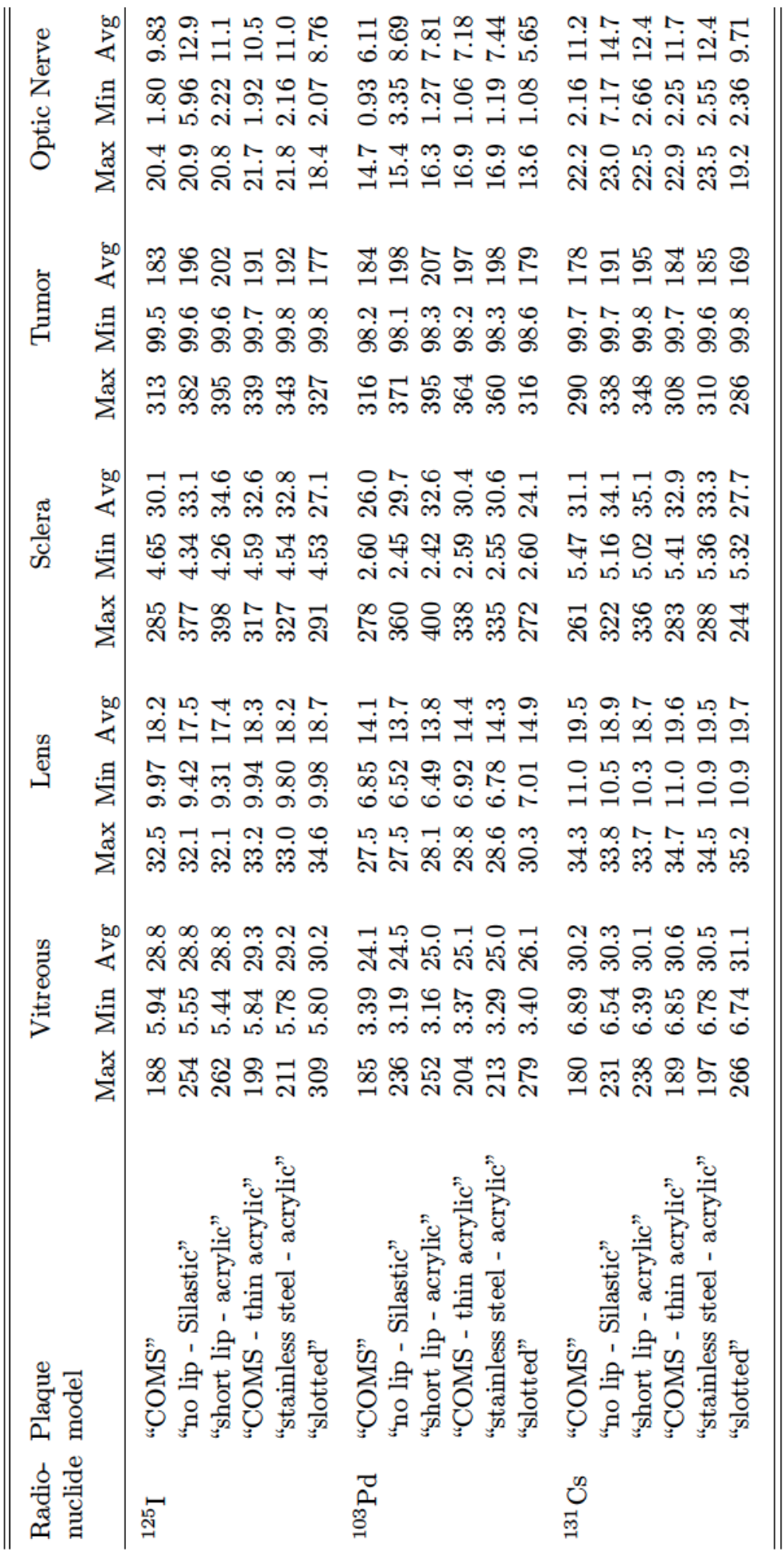



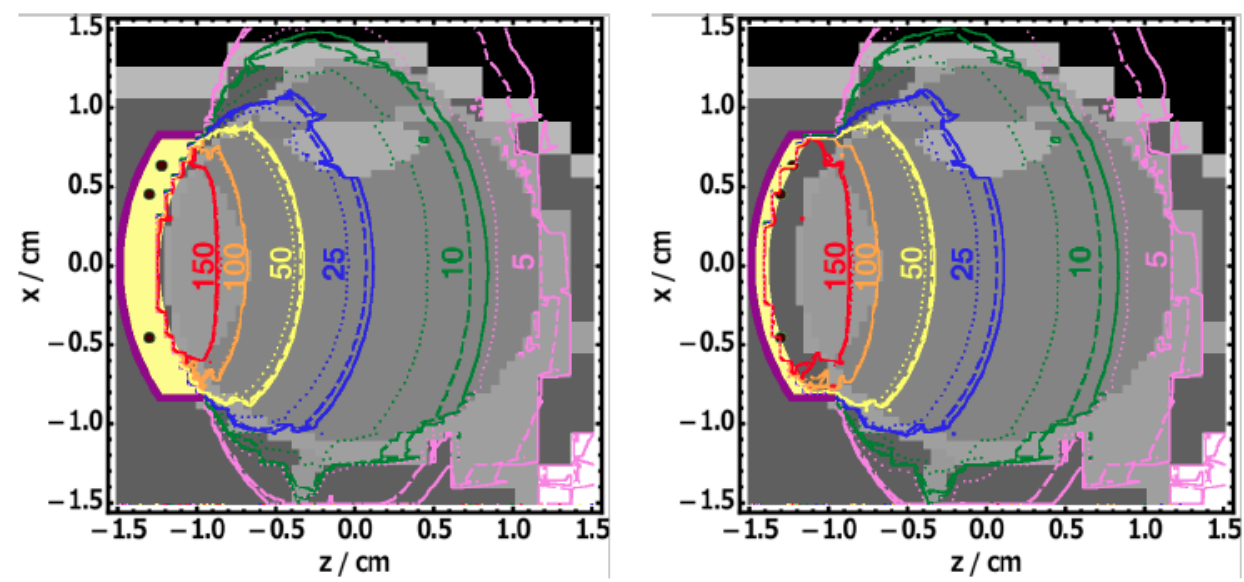

(a) "COMS"

(b) "COMS - thin acrylic"
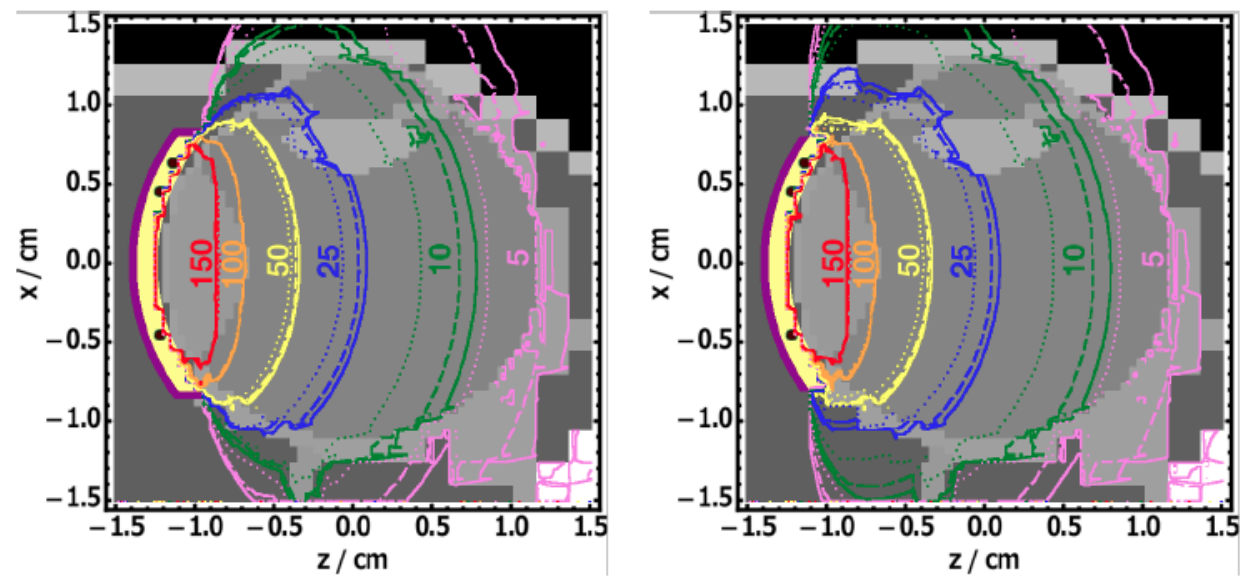

(c) "short lip - acrylic"

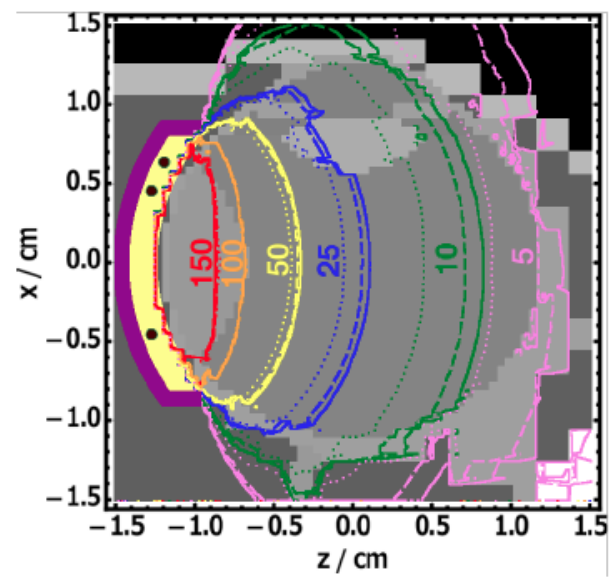

(d) "no lip - Silastic"

(e) "stainless steel - acrylic"

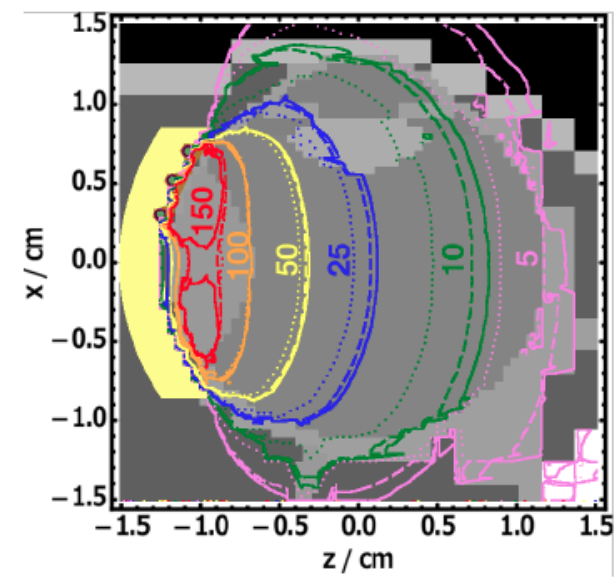

(f) "slotted"

Figure 3.9: Percent isodose $\left(\% \mathrm{D}_{\text {apex }}\right)$ contours from the full eye model simulations for ${ }^{125} \mathrm{I}$ (dashed), ${ }^{103} \mathrm{Pd}$ (dotted), and ${ }^{131} \mathrm{Cs}$ (solid) within each plaque model for the central horizontal slice (voxel centers are at $y=0.025 \mathrm{~cm}$ as voxel boundaries are at $y=0 \mathrm{~cm}$ ).. Although dose is not scored within the plaque, isodose contours intersect the plaque in voxels which are partially occupied by the plaque with centers lying within the plaque. 
Between all the plaque models and all the radionuclides, the largest average $\% \mathrm{D}_{\text {apex }}$ to the tumor is delivered by ${ }^{103} \mathrm{Pd}$ within the "short lip - acrylic" plaque. The lowest average $\% \mathrm{D}_{\text {apex }}$ to the vitreous results from ${ }^{103} \mathrm{Pd}$ within the "COMS" plaque, although the other plaque models are within $2 \% \mathrm{D}_{\text {apex }}$ of its average vitreous $\% \mathrm{D}_{\text {apex }}$ for ${ }^{103} \mathrm{Pd}$. The average and maximum lens $\% \mathrm{D}_{\text {apex }}$ are lowest for ${ }^{103} \mathrm{Pd}$ with the "COMS" plaque (for the average lens dose, the "no lip - Silastic" plaque delivers the same dose) and the maximum optic nerve $\% \mathrm{D}_{\text {apex }}$ is lowest for ${ }^{103} \mathrm{Pd}$ with the "slotted" plaque. For the sclera, the maximum $\% \mathrm{D}_{\text {apex }}$ between all radionuclides and plaque models varies by $156 \% \mathrm{D}_{\text {apex }}$ and is smallest for ${ }^{131} \mathrm{Cs}$ within the "slotted" plaque.

\subsection{Conclusion}

Six representative plaques were modeled in this chapter to investigate the effects of plaque backings and inserts on dose for a range of plaques containing ${ }^{125} \mathrm{I},{ }^{103} \mathrm{Pd}$, and

${ }^{131}$ Cs sources. For both the single seed and the multiseed simulations of each plaque in water, the presence of the plaque affects dose along the plaque axis significantly. The effects of the plaque on dose vary with plaque model and radionuclide and are largest for the "COMS" plaque along most of the plaque axis for all radionuclides studied. Plaque effects on dose differ for single and multiple seed cases in regions near the plaque, and these differences vary with plaque model.

For the multiseed configuration, collimation varies with plaque model. The largest drop in the $\mathrm{D}_{w, w} / \mathrm{D}_{T G-43}$ values between the center and the edge of the plaque in the plane parallel to the plaque face (i.e., perpendicular to the plaque's central axis) occurs for the "slotted" plaque model. Plaques with longer lips have a steeper $\mathrm{D}_{w, w} / \mathrm{D}_{T G-43}$ drop off from the center to the edge of the plaque (in the plane parallel 
to the plaque face) than those with shorter lips or no lips. The presence of a collimating lip can also reduce doses along the plaque axis at the opposite end of the eye compared to the same plaque without the collimating lip.

The combined effects of plaque models and ocular media on dose can be considerable. In general, $\mathrm{D}_{m, m}$ within the tumor is closer to $\mathrm{D}_{T G-43}$ than $\mathrm{D}_{w, w}$, whereas within the majority of the rest of the eye, $\mathrm{D}_{m, m}$ is closer to $\mathrm{D}_{w, w}$ than $\mathrm{D}_{T G-43}$ for all radionuclides. Average $\mathrm{D}_{m, m}$ to the tumor can be higher or lower than $\mathrm{D}_{T G-43}$ depending on the plaque model and radionuclide, with maximum differences between $\mathrm{D}_{m, m}$ and $\mathrm{D}_{T G-43}$ of up to $10 \%$ for ${ }^{125} \mathrm{I}$ and ${ }^{131} \mathrm{Cs}$, and up to $17 \%$ for ${ }^{103} \mathrm{Pd}$. For the lens, all average $\mathrm{D}_{m, m}$ values are reduced compared with $\mathrm{D}_{T G-43}$ values by a maximum of $29 \%$ for ${ }^{125} \mathrm{I}, 35 \%$ for ${ }^{103} \mathrm{Pd}$, and $28 \%$ for ${ }^{131} \mathrm{Cs}$. The effect of each plaque on dose to each structure within the eye model (i.e., the ratio of $\mathrm{D}_{m, m}$ to $\mathrm{D}_{T G-43}$ ) varies between plaque models and radionuclides. When doses for the plaque in the full eye model are normalized to the percent of the apical tumor dose, the largest average $\% \mathrm{D}_{\text {apex }}$ to the tumor is delivered by ${ }^{103} \mathrm{Pd}$ within the "short lip - acrylic" plaque; the lowest $\% \mathrm{D}_{\text {apex }}$ to other ocular structures is generally delivered by ${ }^{103} \mathrm{Pd}$, although the plaque model delivering the lowest dose varies depending on the structure.

Results presented in this chapter depend on the composition and geometry of ocular and plaque media, which are not all accurately known (as discussed in Chapter 2). Further, this chapter employed the same multiseed configuration and plaque size for each plaque model, and in practice these parameters depend on tumor size and vary between institutions. Dose effects have been shown to vary with plaque size, especially in regions near the plaque. ${ }^{44}$ Despite these limitations, work presented in this chapter has shown that doses are highly sensitive to the choice of plaque backing and insert, suggesting the importance of using accurate model-based dose calculations for photon-emitting eye plaque brachytherapy. 


\section{Chapter 4}

\section{Conclusions and outlook}

Dose for brachytherapy is currently calculated using the TG-43 approach for the seeds in water without interseed effects. However, as a result of the low energies used in brachytherapy, interseed attenuation and the presence of heterogeneous non-waterequivalent media has been shown to affect dose significantly. Despite differences found between TG-43 and model-based dose calculations for several brachytherapy applications, studies on the effects on dose of heterogeneous media for eye plaque brachytherapy (including the high- $Z$ plaque backing and ocular media) have been relatively limited. Some groups have investigated plaque effects on dose in water for various plaque models, however the method of dose calculation or measurement, source type, plaque size and type of seed configuration vary between groups, making comparison between doses for each plaque model difficult. Preliminary work on investigating ocular media effects on dose has been performed by some groups (e.g., studying the effects of the air interface or bone in the vicinity of the eye), however these studies have not considered ocular media effects using a full model of the eye and surrounding tissues (including geometry and composition).

This thesis investigated ocular media and plaque effects on dose for ${ }^{125} \mathrm{I},{ }^{103} \mathrm{Pd}$, and ${ }^{131} \mathrm{Cs}$ eye plaque brachytherapy using Monte Carlo simulations for a full eye model and six representative plaque models. In Chapter 2, the representative voxelized eye model developed for this study was presented, including the composition and geometry of ocular media and tissues surrounding the eye. Chapter 2 focused on the effects of ocular media on dose for the widely-used $16 \mathrm{~mm}$ COMS plaque by comparing dose for the plaque in the full eye model and in water to dose for the seeds 
alone in water without interseed effects (TG-43). The presence of ocular media was shown to affect dose significantly due to differences between attenuation coefficients and mass energy absorption coefficients in these media compared with water. Other than the air interface, doses within the eye were relatively insensitive to media which surround the eye. In certain regions of the eye, doses using the full eye model were near those for the plaque in water, whereas in other regions of the eye they were closer to those for the TG-43 simulations. Chapter 2 also compared doses to ocular volumes of interest with the corresponding doses to points of interest to which dose is currently reported. Doses to points were not found to accurately represent the maximum, minimum and average doses to volumes of interest, suggesting that it may be more appropriate to report doses to volumes rather than to points.

Chapter 3 described the development of five representative plaque models for a range of photon-emitting plaques used at various institutions. These plaque models, as well as the COMS plaque, were modeled within a water phantom and the full eye model of Chapter 2 for both single seed and multiseed configurations, and were compared to the same seed configurations in water without the plaque. The effects of each plaque on dose were found to vary considerably and to depend on the choice of radionuclide. Further, differences between the plaque effects on dose for single seed and multiseed simulations varied between plaque models. The combined effects of the plaque and ocular media on dose were also investigated in Chapter 3 for each of the plaque models. Once again, significant variations in doses to ocular structures were observed between plaque models and radionuclides.

The results presented in this work highlight the limitations of the current TG-43 approach to dosimetry in eye plaque brachytherapy, where dose is reported to points of interest for the seeds in water. Accurate determination of ocular dose distributions is integral to understanding the radiobiological effect of eye plaque treatments; ac- 
counting for the effects of the plaque and ocular media on dose is therefore important for improving treatment outcomes. Several challenges exist in implementing modelbased dose calculations for ocular brachytherapy, namely that ocular media compositions are not all accurately known, and ocular media compositions and geometry vary within the population. In order to properly take into account ocular media and plaque effects on dose, it is necessary to improve upon existing knowledge of ocular media compositions and to quantify their variation within the population. It may also be necessary to take into account patient-specific ocular and tumor geometries to calculate doses accurately. Another challenge in implementing model-based ocular brachytherapy dose calculations is the wide variety of plaques used for the treatment of uveal melanoma across institutions, as many types of radionuclides, seed arrangements and plaque shapes/sizes are used, in addition to various plaque backing and insert materials. Model-based dose calculations for eye plaque brachytherapy could therefore be simplified through the standardization of eye plaques (i.e., in terms of the plaque type, seed arrangement etc.), as this would avoid the necessity for each institution to quantify plaque effects for their specific model (which currently are not quantified in a consistent manner between institutions, if at all).

Regardless of improved dosimetry using model-based dose calculations, there may be uncertainty in plaque positioning during treatment despite techniques used to properly position the plaques (e.g., the use of a clear dummy plaque and transillumination to determine tumor location and plaque placement prior to plaque insertion, ${ }^{17}$ and ultrasound imaging to confirm proper plaque placement after its insertion ${ }^{38,90}$ ). The uncertainty associated with plaque placement may limit the benefits of improving eye plaque dosimetry and should therefore be quantified.

This study focused on ocular media and plaque effects for ${ }^{125} \mathrm{I},{ }^{103} \mathrm{Pd}$ and ${ }^{131} \mathrm{Cs}$, three photon-emitting radionuclides. As mentioned in Chapter 1, other photon- 
emitting radionuclides are used, as well as beta-emitting radionuclides. In the future, the full eye model developed within this work might be used to investigate the ocular media and plaque effects for plaques which use beta-emitting radionuclides, as these have not yet been characterized and are expected to be different than for photonemitting radionuclides. Some studies have attempted to correlate dose distributions with treatment outcomes (i.e., local tumor control, loss of vision or other ocular complications, and tumor metastases), ${ }^{33,85,90}$ however these studies have generally been based on the assumption that ocular media are water-equivalent. Future work may therefore involve correlating dose distributions (including maximum, minimum and average doses to ocular structures), as well as other radiobiological factors (e.g., dose rate and implant duration ${ }^{85,92}$ ) with treatment outcomes using an eye model similar to the one developed in this work. 


\section{References}

[1] M. Lesperance, M. Inglis-Whalen, and R. Thomson, Model-based dose calculations for COMS eye plaque brachytherapy, Med. Phys. (in press).

[2] M. Lesperance, M. Martinov, and R. Thomson, Model-based dose calculations for ${ }^{125} \mathrm{I},{ }^{103} \mathrm{Pd}$, and ${ }^{131} \mathrm{Cs}$ eye plaque brachytherapy, submitted to Med. Phys. .

[3] ICRP, Adult Reference Computational Phantoms, ICRP Report 110, ICRP, Washington D.C., 2009.

[4] M. Lesperance, M. Martinov, M. Inglis-Whalen, and R. Thomson, Model-based dose calculations for eye plaque brachytherapy, Med. Phys.(abstract) 40, 476 (2013).

[5] E. B. Podgorsak, editor, Review of Radiation Oncology Physics: A Handbook for Teachers and Students, Educational Report Series, IAEA, Vienna, Austria, 2004.

[6] L. Beaulieu, A. C. Tedgren, J.-F. Carrier, S. D. Davis, F. Mourtada, M. J. Rivard, R. M. Thomson, F. Verhaegen, T. A. Wareing, and J. F. Williamson, Report of the Task Group 186 on model-based dose calculation methods in brachytherapy beyond the TG-43 formalism: Current status and recommendations for clinical implementation, Med. Phys. 39, 6208 - 6236 (2012).

[7] C. Ozhasoglu and M. Murphy, Issues in respiratory motion compensation during external-beam radiotherapy, Int. J. Radiat. Oncol. Biol. Phys. 52, 1389 - 1399 (2002).

[8] P. J. Keall, G. S. Mageras, J. M. Balter, R. S. Emery, K. M. Forster, S. B. Jiang, J. M. Kapatoes, H. D. Kubo, D. A. Low, M. J. Murphy, B. R. Murray, C. R. Ramsey, M. B. V. Herk, S. S. Vedam, J. W. Wong, and E. Yorke, The Management of Respiratory Motion in Radiation Oncology: Report of AAPM Task Group 76, Report 91, AAPM, 2006.

[9] R. Nath, L. L. Anderson, G. Luxton, K. A. Weaver, J. F. Williamson, and A. S. Meigooni, Dosimetry of interstitial brachytherapy sources: Recommendations of the AAPM Radiation Therapy Committee Task Group No. 43, Med. Phys. 22, $209-234$ (1995).

[10] M. J. Rivard, B. M. Coursey, L. A. DeWerd, M. S. Huq, G. S. Ibbott, M. G. Mitch, R. Nath, and J. F. Williamson, Update of AAPM Task Group No. 43 Report: A revised AAPM protocol for brachytherapy dose calculations, Med. Phys. 31, $633-674$ (2004).

[11] H. E. Johns and J. R. Cunningham, The physics of radiology, 4th ed., Charles C. Thomas, Springfield, Illinois, 1983. 
[12] J.-F. Carrier, M. D'Amours, F. Verhaegen, B. Reniers, A.-G. Martin, E. Vigneault, and L. Beaulieu, Postimplant dosimetry using a Monte Carlo dose calculation engine: a new clinical standard, Int. J. Radiat. Oncol. Biol. Phys. $68,1190-1198$ (2007).

[13] R. E. Taylor, Monte Carlo Calculations for Brachytherapy, MSc Thesis, Physics, Carleton University, Ottawa, Ontario (2006).

[14] J. G. H. Sutherland, K. M. Furutani, Y. I. Garces, and R. M. Thomson, Modelbased dose calculations for ${ }^{125}$ I lung brachytherapy, Med. Phys. 39, $4365-4377$ (2012).

[15] C. L. Shields and J. A. Shields, Ocular melanoma: relatively rare but requiring respect, Clinics in Dermatology 27, 122 - 133 (2009).

[16] A. Karvat, C. Duzenli, R. Ma, K. Paton, and T. Pickles, The treatment of choroidal melanoma with Au-198 plaque brachytherapy, Radiother. Oncol. 59, $153-156$ (2001).

[17] K. L. Leonard, N. L. Gagne, J. E. Mignano, J. S. Duker, E. A. Bannon, and M. J. Rivard, A 17-year retrospective study of institutional results for eye plaque brachytherapy of uveal melanoma using ${ }^{125} \mathrm{I},{ }^{103} \mathrm{Pd}$, and ${ }^{131} \mathrm{Cs}$ and historical perspective, Brachytherapy 10, 331 - 339 (2011).

[18] F. Mourtada, N. Koch, and W. Newhauser, ${ }^{106} \mathrm{Ru} /{ }^{106} \mathrm{Rh}$ plaque and proton radiotherapy for ocular melanoma: a comparative dosimetric study, Radiat. Prot. Dosim. 116, $454-460$ (2005).

[19] M. A. Astrahan, A patch source model for treatment planning of ruthenium ophthalmic applicators, Med. Phys. 30, 1219 - 1228 (2003).

[20] S. Nag, J. M. Quivey, J. D. Earle, D. Followill, J. Fontanesi, and P. T. Finger, The American Brachytherapy Society recommendations for brachytherapy of uveal melanomas, Int. J. Radiat. Oncol. Biol. Phys. 56, 544 - 555 (2003).

[21] Collaborative Ocular Melanoma Study Group, The COMS randomized trial of iodine 125 brachytherapy for choroidal melanoma, III: initial mortality findings. COMS report no. 18, Arch. Ophthalmol. 105, 969-982 (2001).

[22] Collaborative Ocular Melanoma Study Group, The COMS randomized trial of iodine 125 brachytherapy for choroidal melanoma: V. Twelve-year mortality rates and prognostic factors: COMS report no. 28, Arch. Ophthalmol. 124, 1684-1693 (2006).

[23] Collaborative Ocular Melanoma Study Group, COMS manual of procedures, Ch 12: Radiation therapy, COMS manual of procedures PB95-179693, Springfield, VA: National Technical Information Service, 1995.

[24] A. Winfried, B. Pothmann, P. Tabor, K. Muskalla, R. Nat., K.-P. Hermann, D. Harder, and P. Nat., Dosimetry and physical treatment planning for iodine eye plaque therapy, Int. J. Radiat. Oncol. Biol. Phys. 20, 1087 - 1092 (1999). 
[25] I. Puusaari, J. Heikkonen, and T. Kivelä, Ocular complications after iodine brachytherapy for large uveal melanomas, Ophthalmology 111, 1768 - 1777 (2004).

[26] S. Nag, D. Wang, H. Wu, C. J. Bauer, R. B. Chambers, and F. H. Davidorf, Custom-made "Nag" eye plaques for ${ }^{125} \mathrm{I}$ brachytherapy, Int. J. Radiat. Oncol. Biol. Phys. 56, $1373-1380$ (2003).

[27] C. Karolis, R. Frost, and F. Billson, A thin I-125 seed eye plaque to treat intraocular tumors using an acrylic insert to precisely position the sources, Int. J. Radiat. Oncol. Biol. Phys. 18, 1209 - 1213 (1990).

[28] D. Granero, J. Pérez-Calatayud, F. Ballester, and E. Casal, Dosimetric study of the 15 mm ROPES eye plaque, Med. Phys. 31, 3330 - 3336 (2004).

[29] H. Zhang, F. Davidorf, and Y. Qi, Comparison of $16 \mathrm{~mm}$ OSU-Nag and COMS eye plaques, J of App Clin Med Phys 13, 166 - 178 (2012).

[30] M. A. Astrahan, A. Szechter, and P. T. Finger, Design and dosimetric considerations of a modified COMS plaque: The reusable "seed-guide" insert, Med. Phys. 32, 2706 - 2716 (2005).

[31] P. T. Finger, A. Berson, and A. Szechter, Palladium-103 plaque radiotherapy for choroidal melanoma, Ophthalmology 106, 606 - 613 (1999).

[32] M. A. Astrahan, G. Luxton, Q. Pu, and Z. Petrovich, Conformal episcleral plaque therapy, Int. J. Radiat. Oncol. Biol. Phys. 39, 505 - 519 (1997).

[33] I. Puusaari, J. Heikkonen, and T. Kivelä, Effect of radiation dose on ocular complications after iodine brachytherapy for large uveal melanoma: empirical data and simulation of collimating plaques, Invest. Ophth. Vis. Sci. 45, 3425 3434 (2004).

[34] C. L. Shields, J. A. Shields, P. De Potter, A. D. Singh, C. Hernandez, and L. W. Brady, Treatment of non-resectable malignant iris tumours with custom designed plaque radiotherapy, Br. J. Ophthalmol. 79, 306 - 312 (1995).

[35] R. M. Thomson, K. M. Furutani, J. S. Pulido, S. L. Stafford, and D. W. O. Rogers, Modified COMS plaques for ${ }^{125} \mathrm{I}$ and ${ }^{103} \mathrm{Pd}$ iris melanoma brachytherapy, Int. J. Radiat. Oncol. Biol. Phys. 78, 1261 - 1269 (2010).

[36] P. T. Finger, Finger's "slotted" eye plaque for radiation therapy: treatment of juxtapapillary and circumpapillary intraocular tumours, Br. J. Ophthalmol. 91, $891-894$ (2007).

[37] D. Flu, G. Anastassiou, J. Wening, W. Sauerwein, and N. Bornfeld, The design and the dosimetry of bi-nuclide radioactive ophthalmic applicators, Med. Phys. $31,1481-1488$ (2004). 
[38] S.-T. Chiu-Tsao, M. A. Astrahan, P. T. Finger, D. S. Followill, A. S. Meigooni, C. S. Melhus, F. Mourtada, M. E. Napolitano, R. Nath, M. J. Rivard, D. W. O. Rogers, and R. M. Thomson, Dosimetry of ${ }^{125} \mathrm{I}$ and ${ }^{103} \mathrm{Pd}$ COMS eye plaques for intraocular tumors: Report of Task Group 129 by the AAPM and ABS, Med. Phys. 39, $6161-6184$ (2012).

[39] H. Zhang, D. Martin, S.-T. Chiu-Tsao, A. Meigooni, and B. R. Thomadsen, A comprehensive dosimetric comparison between ${ }^{131} \mathrm{Cs}$ and ${ }^{125} \mathrm{I}$ brachytherapy sources for COMS eye plaque implant, Brachytherapy 9, 362 - 372 (2010).

[40] R. Behrens, G. Dietze, and M. Zankl, Dose conversion coefficients for electron exposure of the human eye lens, Phys. Med. Biol. 54, 4069 - 4087 (2009).

[41] R. Behrens and G. Dietze, Dose conversion coefficients for photon exposure of the human eye lens, Phys. Med. Biol. 56, 415 - 438 (2011).

[42] A. Peratta, 3D low frequency electromagnetic modelling of the human eye with boundary elements: Application to conductive keratoplasty, Eng. Anal. Bound. Elem. 32, 726 - 735 (2007).

[43] A. de la Zerda, S. Chiu-Tsao, J. Lin, L. L. Boulay, I. Kanna, J. H. Kim, and $\mathrm{H}$. Tsao, ${ }^{125} \mathrm{I}$ plaque dose distribution including penumbra characteristics, Med. Phys. 23, 407 - 418 (1996).

[44] R. M. Thomson, R. E. P. Taylor, and D. W. O. Rogers, Monte Carlo dosimetry for ${ }^{125} \mathrm{I}$ and ${ }^{103} \mathrm{Pd}$ eye plaque brachytherapy, Med. Phys. 35, $5530-5543$ (2008).

[45] M. A. Astrahan, Improved treatment planning for COMS eye plaques, Int. J. Radiat. Oncol. Biol. Phys. 61, 1227 - 1242 (2005).

[46] C. S. Melhus and M. J. Rivard, COMS eye plaque brachytherapy dosimetry simulations for ${ }^{103} \mathrm{Pd},{ }^{125} \mathrm{I}$, and ${ }^{131} \mathrm{Cs}$, Med. Phys. 35, $3364-3371$ (2008).

[47] W. Alberti, Acute and late side effects of radiotherapy for ocular disease: An overview, in Radiotherapy of Ocular Diseases, edited by T. Wiegel, N. Bornfeld, M. H. Foerster, and W. Hinkelbein, pages 281 - 286, S. Karger AG, Basel, 1997.

[48] M. J. Rivard, S.-T. Chiu-Tsao, P. T. Finger, A. S. Meigooni, C. S. Melhus, F. Mourtada, M. E. Napolitano, D. W. O. Rogers, R. M. Thomson, and R. Nath, Comparison of dose calculation methods for brachytherapy of intraocular tumors, Med. Phys. 38, 306 - 316 (2011).

[49] J. Briesmeister, MCNP-A general purpose Monte Carlo code for neutron and photon transport, Version 3A, Los Alamos National Laboratory Report LA7396-M (Los Alamos, NM) (1986).

[50] F. B. Brown (Editor), MCNP - A general Monte Carlo N-particle transport code Version 5, Report LA-UR-03-1987, Los Alamos National Laboratory, Los Alamos, NM, 2003.

[51] S. Agostinelli et al., GEANT4 - a simulation toolkit, Nucl. Instrum. Meth. A 506, $250-303$ (2003). 
[52] R. E. P. Taylor, G. Yegin, and D. W. O. Rogers, Benchmarking BrachyDose: voxel-based EGSnrc Monte Carlo calculations of TG-43 dosimetry parameters, Med. Phys. 34, 445 - 457 (2007).

[53] R. M. Thomson, G. Yegin, R. E. P. Taylor, J. G. H. Sutherland, and D. W. O. Rogers, Fast Monte Carlo dose calculations for brachytherapy with BrachyDose, Med. Phys. 37, 3910 - 3911 (2010) (abstract).

[54] I. Kawrakow, E. Mainegra-Hing, D. W. O. Rogers, F. Tessier, and B. R. B. Walters, The EGSnrc Code System: Monte Carlo simulation of electron and photon transport, NRC Technical Report PIRS-701 v4-2-3-2, National Research Council Canada, Ottawa, Canada. http://www.irs.inms.nrc.ca/inms/irs/EGSnrc/EGSnrc.html, 2011.

[55] G. Yegin, A new approach to geometry modelling of Monte Carlo particle transport: an application to EGS, Nucl. Instrum. Meth. B 211, 331 - 338 (2003).

[56] R. E. P. Taylor and D. W. O. Rogers, EGSnrc Monte Carlo calculated dosimetry parameters for ${ }^{192} \mathrm{Ir}$ and ${ }^{169} \mathrm{Yb}$ brachytherapy sources, Med. Phys. 35, 4933 4944 (2008).

[57] A. K. Carlsson and A. Ahnesjö, The collapsed cone superposition algorithm applied to scatter dose calculations in brachytherapy, Med. Phys. 27, $2320-$ $2332(2000)$.

[58] A. K. Carlsson and A. Ahnesjö, Point kernels and superposition methods for scatter dose calculations in brachytherapy, Phys. Med. Biol. 45, 357-382 (2000).

[59] K. R. Russell and A. Ahnesjö, Dose calculation in brachytherapy for a source using a primary and scatter dose separation technique, Phys. Med. Biol. 41, 1007 - 1024 (1996).

[60] R. E. P. Taylor and D. W. O. Rogers, More accurate fitting of ${ }^{125} \mathrm{I}$ and ${ }^{103} \mathrm{Pd}$ radial dose functions, Med. Phys. 35, 4242 - 4250 (2008).

[61] R. E. P. Taylor and D. W. O. Rogers, An EGSnrc Monte Carlo-calculated database of TG-43 parameters, Med. Phys. 35, 4228 - 4241 (2008).

[62] R. E. P. Taylor, G. Yegin, and D. W. O. Rogers, Monte Carlo modeling of the Xoft Axxent x-ray source, Med. Phys. 33, 2205 (abs) (2006).

[63] M. J. Rivard, S. D. Davis, L. A. DeWerd, T. W. Rusch, and S. Axelrod, Calculated and measured brachytherapy dosimetry parameters in water for the Xoft Axxent X-Ray Source: An electronic brachytherapy source, Med. Phys. 33, 4020 $-4032(2006)$.

[64] M. J. Hogan, J. A. Alvarado, and J. E. Weddell, Histology of the human eye: an atlas and textbook, W. B. Saunders Company, Philadelphia, 1971.

[65] F. A. Jakobiec, Ocular Anatomy, Embryology and Teratology, Harper \& Row, Philadelphia, 1982. 
[66] H. Davson, The Eye, 3rd ed., Academic Press, Inc., Orlando, FL, 1984.

[67] N. K. Tumram, R. V. Bardale, and A. P. Dongre, Postmortem analysis of synovial fluid and vitreous humour for determination of death interval: A comparative study, Forensic Sci. Int. 204, 186 - 190 (2011).

[68] E. R. Berman, Biochemistry of the Eye., Plenum, New York, 1991.

[69] ICRU, Photon, Electron, Photon and Neutron Interaction Data for Body Tissues, ICRU Report 46, ICRU, Washington D.C., 1992.

[70] M. J. Berger and J. H. Hubbell, XCOM: Photon cross sections on a personal computer, Report NBSIR87-3597, National Institute of Standards Technology (NIST), Gaithersburg, MD 20899, U.S.A., 1987.

[71] K. Meek, The Cornea and Sclera, in Collagen: Structure and Mechanics, edited by P. Fratzl, pages 359 - 396, Springer, New York, 2008.

[72] H. Q. Woodard and D. R. White, The composition of body tissues, Brit. J. Radiol. 59, 1209 - 1219 (1986).

[73] R. L. Maughan, P. Chuba, A. T. Porter, E. Ben-Josef, D. R. Lucas, and B. E. Bjarngard, Mass energy-absorption coefficients and mass collision stopping powers for electrons in tumors of various histologies, Med. Phys. 26, $472-477$ (1999).

[74] B. Brodsky, J. A. Werkmeister, and J. A. M. Ramshaw, Collagens and Gelatins, in Biopolymers, edited by A. Steinbuchel, pages 119 - 147, Wiley-VCH, Weinheim, Germany, 2003.

[75] K. Zierold, J. Michel, C. Terryn, and G. Balossier, The distribution of light elements in biological cells measured by electron probe X-ray microanalysis of cryosections, Microsc. Microanal. 11, 138 - 145 (2005).

[76] ICRP, Basic Anatomical and Physiological Data for Use in Radiological Protection: Reference Values, Annals of the ICRP 89, ICRP, Washington D.C., 2003.

[77] A. Presland, Applied ocular physiology and anatomy, Anaesth. Intens. Care 8, $379-382$ (2007).

[78] B. R. B. Walters, I. Kawrakow, and D. W. O. Rogers, DOSXYZnrc Users Manual, NRC Technical Report PIRS-794 (rev B), Ottawa, Canada, 2005.

[79] M. J. Rivard, Brachytherapy dosimetry parameters calculated for a ${ }^{131} \mathrm{Cs}$ source, Med. Phys. 34, $754-762$ (2007).

[80] M. J. Rivard, C. S. Melhus, S. Sioshansi, and J. Morr, The impact of prescription depth, dose rate, plaque size, and source loading on the central axis using ${ }^{103} \mathrm{Pd}$, ${ }^{125} \mathrm{I}$, and ${ }^{131} \mathrm{Cs}$, Brachytherapy 7, $327-335$ (2008). 
[81] R. E. P. Taylor and D. W. O. Rogers, The Carleton Laboratory for Radiotherapy Physics TG-43 Parameter Database, http://www.physics.carleton.ca/clrp/seed_database .

[82] D. R. White, H. Q. Woodard, and S. M. Hammond, Average soft-tissue and bone models for use in radiation dosimetry, Brit. J. Radiol. 60, 907 - 913 (1987).

[83] M. Charles and N. Brown, Dimensions of the human eye relevant to radiation protection (dosimetry), Phys. Med. Biol. 20, 202 - 218 (1975).

[84] A. Bonfiglio, R. Repetto, J. H. Siggers, and A. Stocchino, Investigation of the motion of a viscous fluid in the vitreous cavity induced by eye rotations and implications for drug delivery, Phys. Med. Biol. 58, 1969 - 1982 (2013).

[85] P. T. Finger, Radiation therapy for orbital tumors: concepts, current use, and ophthalmic radiation side effects, Surv. Ophthalmol. 54, 545 - 568 (2009).

[86] S. Chiu-Tsao, L. L. Anderson, K. O'Brien, L. Stabile, and J. C. Liu, Dosimetry for ${ }^{125}$ I seed (model 6711) in eye plaques, Med. Phys. 20, 383 - 389 (1993).

[87] W. Alberti, B. Pothmann, P. Tabor, K. Muskalla, K.-P. Hermann, and D. Harder, Dosimetry and physical treatment planning for iodine eye plaque therapy, Int. J. Radiat. Oncol. Biol. Phys. 20, 1087 - 1092 (1991).

[88] A. Wu and F. Krasin, Film dosimetry analyses on the effect of gold shielding for Iodine-125 eye plaque therapy for choroidal melanoma, Med. Phys. 17, 843 - 846 (1990).

[89] ICRU, Stopping powers for electrons and positrons, ICRU Report 37, ICRU, Bethesda, MD., 1984.

[90] P. T. Finger, K. J. Chin, G.-P. Yu, and N. S. Patel, Risk factors for cataract after Palladium-103 ophthalmic plaque radiation therapy, Int. J. Radiat. Oncol. Biol. Phys. 80, 800 - 806 (2011).

[91] M. Astrahan, G. Luxton, G. Jozsef, T. D. Kampp, P. Liggett, and M. D. Sapozink, An interactive treatement planning system for ophthalmic plaque radiotherapy, Int. J. Radiat. Oncol. Biol. Phys. 18, 679 - 687 (1990).

[92] N. L. Gagne, K. L. Leonard, and M. J. Rivard, Radiobiology for eye plaque brachytherapy and evaluation of implant duration and radionuclide choice using an objective function, Med. Phys. 39, 3332 - 3342 (2012). 